UB-HET-93-1

August 1993

\title{
Chromo-electroweak interference and parity-violating asymmetries in the production of an electroweak boson + two jets in hadron collisions
}

\author{
Richard J. Gonsalves \\ Department of Physics \\ State University of New York at Buffalo \\ Buffalo, New York 14260, USA \\ C.F. Wai \\ Institute of Physics \\ Academia Sinica \\ Taipei, Taiwan 11529, Republic of China \\ [Published in Phys. Rev. D 49 190-218 (1994); Erratum: ibid., D 511428 (1995)]

\begin{abstract}
In the Standard Model the process $q \bar{q} \rightarrow q \bar{q} V$ (where $V=W^{ \pm}, Z^{0}$ or $\gamma$ ) can occur via gluon exchange and also via $W^{ \pm}$or $Z^{0}$ exchange. The corresponding chromodynamic and electroweak amplitudes can interfere with one another. These interference cross sections are largest when the exchanged $W^{ \pm}$or $Z^{0}$ is on-shell when they are also odd under parity. Interference cross sections computed using helicity-amplitude techniques are presented for all interesting subprocesses as well as for the processes $q \bar{q} \rightarrow q \bar{q} l \bar{l}$ in which the lepton pair $l \bar{l}$ comes from the decay of $V$ on-shell. Parity-violating asymmetries are defined and presented at the parton level and for the hadronic processes $p p$ or $p \bar{p} \rightarrow V$ +2 jets or $l \bar{l}+2$ jets. These asymmetries are independent of the polarizations of all particles involved, and do not require that the flavors of the jet partons be measured. They are generally of order $0.01 \mathrm{pb}$ at energies $\sqrt{s} \gtrsim 1 \mathrm{TeV}$.
\end{abstract}

12.10.Dm, 13.85.Qk, 14.80.Er

Typeset using REVTEX 


\section{INTRODUCTION}

The Standard $\mathrm{SU}(3)_{\mathrm{c}} \otimes \mathrm{SU}(2)_{\mathrm{L}} \otimes \mathrm{U}(1)$ Model [1,2] of the strong and electroweak interactions has several features for which a simple and satisfactory theoretical explanation is lacking. These include the origin of the masses of the $W$ and $Z$ bosons, and the pattern of the couplings of these bosons to quarks and leptons - a pattern which involves several unexplained parameters and violation of symmetries such as parity and possibly CP conjugation. Experiments which study the production and decay of one or more electroweak gauge bosons might provide clues that will enable us to better understand these puzzling features of the Standard Model. In this paper we will present some intriguing predictions of the Standard Model for correlations between the final state particles in the production of pairs of electroweak bosons in hadron-hadron collisions. These correlations produce asymmetries in the predicted cross sections that are very sensitive to the details of the couplings of the gauge bosons to quarks and in particular to the parity-violating nature of these couplings. While these asymmetries are predicted to be rather small, their measurement would provide an elegant test of the Standard Model, and deviations from these predictions might provide clues to a more fundamental structure which might underlie the Standard Model.

The parity-violating asymmetries presented in this paper owe their existence to a quantum mechanical interference between amplitudes involving the exchange of gluons and electroweak bosons in $q \bar{q}$ annihilation. This phenomenon of "chromo-electroweak interference" is exemplified by the diagrams in Fig. 11. It is similar in nature to the interference [3] between a virtual photon and a virtual $Z^{0}$ which gives rise for example to the well known asymmetry [4] in $e^{+} e^{-} \rightarrow \mu^{+} \mu^{-}$, to the parity-violating asymmetry in the scattering of polarized electrons from nuclei [5], or to the parity-violation observed in atomic systems [6]. Unlike electroweak interference between a photon and a $Z^{0}$, which have the same quantum numbers, chromo-electroweak interference between a gluon and an electroweak boson, which have different color and flavor quantum numbers, can occur only if the gluon and the electroweak boson are exchanged in different channels as shown in Fig. 1. An interference between the two diagrams in this figure is interesting because the magnitude of its contribution to the cross section might be expected to lie in between that of the strong and the weak exchange diagrams taken separately: thus if the weak contribution were too small to be observed, the interference might provide the only observable signature of the weak exchange. An extremely interesting possibility arises if at least one of the quarks is polarized and the electroweak boson is a $W$ or a $Z^{0}$, both of which couple asymmetrically to left- and right-handed fermions: the interference contributions can then contain terms that are odd under parity, i.e., that are proportional to invariants such as $\vec{s} \cdot \vec{p}$ where $\vec{s}$ is a spin and $\vec{p}$ a momentum. This possibility has been exploited [7] to make predictions for parity-violating signatures in inclusive hadron production at large $p_{T}$ in polarized hadron-hadron scattering. Unfortunately, experiments with polarized beams are difficult to perform except at low

energies at which the weak couplings are very small, and the prospects for observing these asymmetries do not appear to be very promising.

The prospects for observing chromo-electroweak interference effects might be considerably better at energies above the threshold for producing $W$ and $Z$ bosons. At these energies the weak couplings are in fact only an order of magnitude smaller than the strong interaction coupling. In addition, the cross sections for observing multiparticle final states including jets 
of hadrons at large $p_{T}$ become appreciable at these energies, and this makes it possible to avoid the need for observing polarizations in order to have parity violation. It was pointed out in [8] that if for example a $W$ boson were radiated off one of the quarks lines in Fig. 1, the interference cross section would be parity-violating even if all particles involved were unpolarized. With two incoming particles and three particles in the final state, it becomes possible to construct a non-vanishing parity-odd invariant $\epsilon_{\mu \nu \lambda \sigma} p_{1}^{\mu} p_{2}^{\nu} p_{3}^{\lambda} p_{4}^{\sigma}$ from the momenta $p_{i}$ of the five particles involved, four of which can be chosen to be linearly independent. In [8], it was shown that this interference gave rise to parity-violating asymmetries of order $0.01 \mathrm{pb}$ in the process $p \bar{p} \rightarrow W^{-}+2$ jets at energies $\gtrsim 1 \mathrm{TeV}$. The asymmetry is largest when the electroweak boson in Fig. 1 and the additional radiated $W$ are on-shell. Observation of this asymmetry does not require that the flavors of the jets be determined - only that their momenta be determined with sufficient accuracy to select pairs of jets with the invariant mass of the pair equal to that of a $W$ or a $Z$ within a few GeV. Asymmetries with a similar origin have been studied [9] in $e^{+} e^{-}$annihilation, and an application to the process $e^{+} e^{-} \rightarrow e^{+} e^{-} \gamma$ has recently been discussed in [10]. Similar parity-violating asymmetries occur in the process hadron + hadron $\rightarrow 3$ jets [11]: they originate from diagrams in which the electroweak boson $V$ is replaced by a gluon; they are considerably larger than the asymmetries in $V+2$ jet production discussed in this paper, but may not be easy to detect above the QCD 3-Jet background.

In [8], the radiated boson was taken to be a $W^{-}$and the boson which decayed to 2 jets a $Z^{0}$. The purpose of this paper is to present a detailed derivation and discussion of the results which were summarized in [8], and to extend these results to all possible combinations of pairs of electroweak bosons, including real photons, that appear to be of phenomenological interest. We also consider the more general process in which the final state contains a lepton pair which comes from the decay of an on-shell $W^{ \pm}$or $Z^{0}$, and a $q \bar{q}$ pair which comes from the decay of a second on-shell $W$ or $Z$. In section II, we single out the dominant amplitudes that are expected to contribute to chromo-electroweak interference, and present compact expressions for amplitudes and interference cross sections [12 derived using the powerful and elegant helicity-amplitude techniques [13 that have recently gained popularity. In section III we first define parity-violating asymmetries in a way that depends only on the momenta of the jet partons involved and not on their internal quantum numbers. We then present numerical predictions for these asymmetries, both at the parton and at the hadron levels, first for $V+2$ jet final states where $V=W^{ \pm}, Z^{0}$ or a real photon, and then for $l \bar{l}$ +2 jet final states in which the lepton pair comes from the decay of an on-shell $W^{ \pm}$or $Z^{0}$. We also discuss the problem of observing parity-violating asymmetries above a background 114 that is expected to be as much as two orders of magnitude larger than the signal. Our conclusions are presented in section IV, and some details pertaining to the calculation of the helicity amplitudes and cross sections are presented in the Appendixes.

\section{CHROMO-ELECTROWEAK INTERFERENCE}

\section{A. Subprocesses contributing to $h_{1}+h_{2} \rightarrow j_{1}+j_{2}+V$}

Consider a collision (Fig. 2) of two hadrons $h_{1}$ and $h_{2}$ which produces an electroweak boson $V$ and two well-defined hadron jets $j_{1}$ and $j_{2}$. $V$ stands for one of $W^{+}, W^{-}, Z^{0}$ or $\gamma$. 
$V, j_{1}$ and $j_{2}$ have transverse momenta, with respect to the beam axis, of several GeV. Since $M_{V} / \Gamma_{V} \simeq 30$ for $W^{ \pm}, Z^{0}$, the cross section is enhanced by a factor of $10^{3}$ for on-shell $W$ 's or Z's, relative to off-shell production: we will restrict our attention to this experimentally interesting case. We will also consider production of energetic real photons. The dominant parton hard-scattering processes which lead to this final state are of the form

$$
a_{1}+a_{2} \rightarrow a_{3}+a_{4}+V,
$$

where the $a$ 's stand for various allowed combinations of quarks, antiquarks and gluons. The $\mathcal{O}\left(e g^{2}\right)$ contributions to the hard scattering can be obtained from the generic diagrams of Fig. 2. 3. Here, $e^{2}=4 \pi \alpha$ and $g^{2}=4 \pi \alpha_{s}$. Following Refs. [13], we use the convention that all external particles in a generic diagram are taken to be outgoing. Physical amplitudes are obtained from generic amplitudes by choosing two of the outgoing partons and crossing them to the initial state. These amplitudes yield a cross section of $\mathcal{O}\left(\alpha \alpha_{s}^{2}\right)$.

In addition to these "chromodynamic" or "QCD" amplitudes, there are "electroweak" contributions to the process (2.1). For example, replacing the virtual gluon in Fig. Ba by an electroweak boson $V_{q}$ yields an amplitude of $\mathcal{O}\left(e^{3}\right)$. All such amplitudes can be obtained from the generic diagrams of Fig. $⿴$. The electroweak cross section is suppressed by a factor $\left(\alpha / \alpha_{s}\right)^{2} \simeq 200$ (for $\left.\alpha_{s} \simeq 0.1\right)$. However if $V_{q}\left(=W^{ \pm}, Z^{0}\right)$ is on shell as can happen in

$$
\begin{aligned}
q+\bar{q} \rightarrow & V_{q}+V \\
& \downarrow \\
& q+\bar{q},
\end{aligned}
$$

the cross section is enhanced by a factor $\left(\Gamma_{V_{q}} / M_{V_{q}}\right)^{2} \simeq 10^{3}$. Thus, one might expect the pair-production of on-shell electroweak bosons to produce an easily observable resonance peak above the QCD background in the process (2.1). Unfortunately, this expectation is not borne out by detailed analysis [14]. There are many more QCD diagrams in Fig. (3) than electroweak diagrams in Fig. 团. In particular, gluons can contribute to the QCD cross section, as can various combinations of quarks and antiquarks, while on-shell electroweak-boson pair production can only occur via $q \bar{q}$ annihilation. In addition, the "abelian" and "non-abelian" amplitudes in Fig. 1 tend to contribute with opposite signs since the renormalizability of the theory requires such cancellations in the tree amplitudes at high energies.

In Ref. [8] it was pointed out that the amplitudes in Fig. [1 can interfere with those in Fig. Ba to give contributions to the cross section of $\mathcal{O}\left(\alpha^{2} \alpha_{s}\right)$. Once again, the electroweak contributions of Fig. 1 will be very small unless $V_{q}$ is almost on shell when its propagator will behave like

$$
\frac{1}{k^{2}-M_{V_{q}}^{2}+i \Gamma_{V_{q}} M_{V_{q}}} \simeq \frac{-i}{\Gamma_{V_{q}} M_{V_{q}}} .
$$

Comparing this with the propagator of the gluon in Fig. 邽 which we can assume has roughly the same momentum

$$
\frac{1}{k^{2}+i \epsilon} \simeq-\frac{1}{M_{V_{q}}^{2}},
$$

we see that the QCD background, the interference contribution, and the electroweak pairproduction cross sections are nominally of relative magnitudes 


$$
1:\left(\frac{\alpha}{\alpha_{s}}\right)\left(\frac{M_{V_{q}}}{\Gamma_{V_{q}}}\right):\left(\frac{\alpha}{\alpha_{s}}\right)^{2}\left(\frac{M_{V_{q}}}{\Gamma_{V_{q}}}\right)^{2},
$$

with $\left(\alpha / \alpha_{s}\right)\left(M_{V_{q}} / \Gamma_{V_{q}}\right) \simeq 2.5$. The interference contributions might be expected to be suppressed on account of the following factors: (i) Like the $V-V_{q}$ pair production cross section, there are many fewer initial states than contribute to the QCD background. (ii) Correlations between initial and final parton colors, flavors and helicities further restrict the subprocesses that can receive interference contributions. (iii) Gauge cancellations between the abelian and non-abelian diagrams in Fig. $⿴$ will also tend to suppress the interference contributions. There is one more factor that might, at first sight, actually seem to make the interference terms vanish, and that is the relative factor of $i$ between Eqs. (2.3) and (2.4) when $V_{q}$ is exactly on shell: since the diagrams in Figs. Ba and 4 have the same number of vertices and propagators, the amplitudes would indeed be $90^{\circ}$ out of phase were it not for spinorial factors in the amplitudes that supply a compensating factor of $i$ that comes from spinor traces such as

$$
\operatorname{Tr}\left[\not k_{1} \not k_{2} \not k_{3} \not k_{4} \gamma_{5}\right]=4 i \epsilon_{\mu \nu \lambda \sigma} k_{1}^{\mu} k_{2}^{\nu} k_{3}^{\lambda} k_{4}^{\sigma},
$$

in the spin-averaged cross section. In fact, the interference cross sections presented in Sec. \I9 are all proportional to parity-violating invariants such as that in Eq. (2.6). We will show that, in contrast to the electroweak pair production cross section which is parity conserving, the interference terms give rise to parity-violating asymmetries which might make them observable in spite of the fact that the magnitudes of the interference cross sections (like the electroweak pair production cross sections) are much smaller than the QCD background.

Figure 5 shows the three electroweak amplitudes $E_{1-3}$ which produce an on-shell boson $V_{l} \equiv V$ shown decaying to a lepton-antilepton pair with invariant mass $\left(k_{5}+k_{6}\right)^{2}=M_{V_{l}}^{2}$, and an on-shell boson $V_{q}$ which decays to a quark-antiquark pair with invariant mass $\left(k_{3}+k_{4}\right)^{2}=$ $M_{V_{q}}^{2}$. The quark with momentum $k_{1}$ and the antiquark with momentum $k_{2}$ are to be crossed to the initial state where they represent the annihilating antiquark and quark respectively. The figure also shows the four QCD amplitudes $G_{1-4}$ which are capable of interfering with $E_{1-3}$. Note that, when the particles 1 and 2 are crossed to the initial state, the gluon in amplitudes $G_{1-4}$ has space-like momentum: as in Fig. 1, this is necessary in order that flavor and color quantum numbers of the initial and final quarks in $E_{1-3}$ be the same as in $G_{1-4}$. We will assume that all quarks are massless. Helicity, then, is conserved at each interaction vertex since the electroweak bosons and the gluons are vector bosons. Thus the helicities of the initial and final quarks are the same in the interference, as are the helicities of the antiquarks.

It is important to remember that the Feynman rules require a relative minus sign due to Fermi statistics between the amplitudes $E_{1-3}$ and $G_{1-4}$ since the quark lines differ by the interchange of a pair of labels. This can also be seen by noting that the electroweak pair-production cross section $\left|E_{1-3}\right|^{2}$ is got from a cut-diagram with two quark loops, while the interference $\operatorname{Re}\left(E_{1-3} G_{1-4}^{*}\right)$ is got from a cut-diagram which has a single quark loop.

\footnotetext{
${ }^{1}$ Because initial- and final-state helicities, flavors and colors are correlated, we expect the interference cross section to be suppressed by roughly a factor of $2 n_{c}\left(n_{f} / 2\right)$ relative to the electroweak pair-production cross section.
} 


\section{B. Helicity Amplitudes for $q+\bar{q} \rightarrow q+\bar{q}+V$}

Helicity decomposition techniques [13] have proven to be extremely useful in evaluating tree diagram amplitudes and cross sections. If all external particles in a diagram are massless fermions and all interactions are mediated by vector bosons, the allowed combinations of helicities of the external particles are restricted since helicity is conserved at each interaction vertex along any fermion line. By judiciously choosing the phases of the wavefunctions of the external particles, one can obtain very compact expressions for the independent helicity amplitudes. A spin-averaged cross section can then be obtained by squaring these compact expressions and summing over the allowed helicity combinations. In practice, this procedure yields, with considerably less calculational effort, expressions for cross sections that are more compact than those obtained by traditional trace-algebra techniques in which the spinaveraged cross sections are represented by traces of products of Dirac-matrices. Helicity amplitude techniques are also extremely useful in calculating amplitudes involving external massless vector bosons such as photons and gluons. They can also be employed if the external particles are massive, but the expressions obtained are generally more complex than in the case of massless particles.

In this section, we will present helicity amplitudes for the diagrams in Fig. 5. These amplitudes will be used to evaluate interference contributions to the spin-averaged cross sections in the next section. Diagrams $E_{1-3}$ were considered in [15], where amplitudes and cross sections are presented for electroweak boson pair production with both bosons on shell. To establish notation and conventions, we list the Feynman rules for vertices at which a quark-antiquark pair and a gauge boson are produced:

$$
\begin{aligned}
W^{-}: & -i e Q_{W} U_{u \bar{d}} \delta_{c \bar{c}} \gamma^{\mu} \gamma_{L} \\
W^{+}: & -i e Q_{W} U_{d \bar{u}}^{*} \delta_{c \bar{c}} \gamma^{\mu} \gamma_{L} \\
Z^{0}: & -i e \delta_{f \bar{f}} \delta_{c \bar{c}} \gamma^{\mu}\left(L_{f} \gamma_{L}+R_{f} \gamma_{R}\right) \\
\gamma & :-i e Q_{f} \delta_{f \bar{f}} \delta_{c \bar{c}} \gamma^{\mu} \\
\text { Gluon }: & -i g \delta_{f \bar{f}}\left(\frac{\lambda_{a}}{2}\right)_{c \bar{c}} \gamma^{\mu} .
\end{aligned}
$$

Here, $f=u$ or $d$ is a flavor index, and $u(d)$ are generic labels for weak $I_{z}=\frac{1}{2}\left(-\frac{1}{2}\right)$ quark flavors. $U$ is the Kobayashi-Maskawa matrix, and with $\theta_{W}$ being the weak mixing angle we define

$$
\begin{gathered}
Q_{W}=\frac{1}{\sqrt{2} \sin \theta_{W}}, Q_{u}=\frac{2}{3}, Q_{d}=-\frac{1}{3}, \tau_{u}=1, \tau_{d}=-1 \\
L_{f}=\frac{\tau_{f}}{\sin \left(2 \theta_{W}\right)}-Q_{f} \tan \theta_{W}, R_{f}=-Q_{f} \tan \theta_{W}
\end{gathered}
$$

These definitions can be extended to include couplings to leptons in an obvious way, i.e., $Q_{e^{-}}=-1, Q_{\nu}=0, \tau_{e^{-}}=-1, \tau_{\nu}=+1, U_{\nu e^{+}}=U_{e^{-} \bar{\nu}}=1$, etc. We will often abbreviate $L_{f_{1}}$ by $L_{1}, U_{u_{3} d_{\overline{4}}}$ by $U_{34}$, etc., when the meaning of the abbreviated indices is clear from the context. The index $c$ is a quark color label, and $\operatorname{Tr}\left(\lambda_{a} \lambda_{a}\right)=4 n_{c} C_{F}$, with $n_{c}=3$ and $C_{F}=\frac{4}{3}$. Finally, $\gamma_{L}=\left(1-\gamma_{5}\right) / 2$ and $\gamma_{R}=\left(1+\gamma_{5}\right) / 2$. The Feynman rule for the triple boson vertex is determined by $S U(2)_{L}$ symmetry. 
We next introduce a convenient notation for the wave functions of massless fermions and spinor products that can be constructed from these wavefunctions. An outgoing massless fermion with momentum $k_{i}$ and helicity $\lambda_{i}= \pm \frac{1}{2}$, where $i=1,2,3, \ldots$, will be represented by the symbol $\langle i \pm|$, and an outgoing antifermion with momentum $k_{j}$ and helicity $\lambda_{j}=\mp \frac{1}{2}$ by $|j \pm\rangle$. Conventions for crossing and for evaluating spinor products such as $\langle i+\mid j-\rangle$ are explained in Appendix A. It is rather remarkable that all of the spinor factors in the diagrams of Fig. 5 can be expressed in terms of a single function

$$
F_{123456} \equiv 4\langle 1-\mid 3+\rangle\langle 6+\mid 2-\rangle[\langle 4+\mid 1-\rangle\langle 1-\mid 5+\rangle+\langle 4+\mid 3-\rangle\langle 3-\mid 5+\rangle]
$$

of the outgoing momenta $k_{i}, i=1, \ldots, 6$, which satisfy momentum conservation $\sum_{i=1}^{6} k_{i}=0$. This function was introduced in [15]: some useful properties connected with it are discussed in Appendix B. In the following subsections we present amplitudes for the sums $E \equiv \sum_{i=1}^{3} E_{i}$ and $G \equiv \sum_{i=1}^{4} G_{i}$ of the electroweak and QCD diagrams. These sums are gauge invariant if the bosons $V_{l}$ and $V_{q}$ are on shell. By conservation of helicity, $\lambda_{2}=-\lambda_{1}, \lambda_{4}=-\lambda_{3}$, $\lambda_{6}=-\lambda_{5}$, in $E$, and $\lambda_{2}=-\lambda_{3}, \lambda_{4}=-\lambda_{1}, \lambda_{6}=-\lambda_{5}$, in $G$ : thus, an amplitude is completely specified by $k_{i}$ and $\lambda_{1}, \lambda_{3}$, and $\lambda_{5}$, and we will therefore label the sums $E$ and $G$ with a triplet of signs $\left(\operatorname{sign}\left(\lambda_{1}\right), \operatorname{sign}\left(\lambda_{3}\right), \operatorname{sign}\left(\lambda_{5}\right)\right)$. The on-shell vector bosons involved will be specified by superscripts as follows: $E^{V_{l} V_{q}}$ and $G^{V_{l}}$.

Finally, we define invariants

$$
\begin{gathered}
s_{i j} \equiv\left(k_{i}+k_{j}\right)^{2}, \quad s_{i j l} \equiv\left(k_{i}+k_{j}+k_{l}\right)^{2}, \\
x_{i j l m} \equiv \epsilon_{\mu \nu \lambda \sigma} k_{i}^{\mu} k_{j}^{\nu} k_{l}^{\lambda} k_{m}^{\sigma},
\end{gathered}
$$

where the metric has signature $(+,-,-,-)$ and $\epsilon_{0123}=+1$. We also define a function

$$
D_{i j}^{V} \equiv s_{i j}-M_{V}^{2}+i M_{V} \Gamma_{V}
$$

which occurs in boson propagator denominators.

\section{Amplitudes for $V_{l}=W^{ \pm}, V_{q}=W^{\mp}$}

Since $W^{ \pm}$only couple to left handed fermions, the non-zero helicity amplitudes are $E_{1,2}(-,-,-), E_{3}(\mp,-,-), G_{1,2}(-, \mp,-)$, and $G_{3,4}(\mp,-,-)$. However, since $\lambda_{2}=-\lambda_{1}$ and $\lambda_{4}=-\lambda_{3}$ in $E_{i}$ while $\lambda_{2}=-\lambda_{3}$ and $\lambda_{4}=-\lambda_{1}$ in $G_{i}$, only the amplitudes $E(-,-,-)$ and $G(-,-,-)$ will contribute to chromo-electroweak interference. Following [15] we find that the electroweak amplitude for $V_{l}=W^{-}, V_{q}=W^{+}$is

$$
\begin{aligned}
E^{W^{-} W^{+}}(-,-,-)=\frac{K_{e} Q_{W}^{2} U_{34} U_{65}^{*} \delta_{12}}{D_{34}^{W} D_{56}^{W}} & \\
\times & {\left[-\frac{\left(1+\tau_{1}\right) Q_{W}^{2}}{2 s_{234}} F_{125634}-\frac{\left(1-\tau_{1}\right) Q_{W}^{2}}{2 s_{134}} F_{123456}\right.} \\
& \left.+\left(\frac{L_{1} \cot \theta_{W}}{D_{12}^{Z}}+\frac{Q_{1}}{D_{12}^{\gamma}}\right)\left(F_{123456}-F_{125634}\right)\right],
\end{aligned}
$$

where 


$$
K_{e}=i e^{4} \delta_{c_{1} c_{2}} \delta_{c_{3} c_{4}}
$$

We note that $\delta_{12} \equiv \delta_{f_{1} f_{2}}$, i.e., flavors $f_{1}=u, d$ are both allowed. The factor $U_{34}$ implies that $f_{3}=u$ and $f_{4}=\bar{d}$. Further, for the kinematic configurations of interest, $D_{34}^{W}=D_{56}^{W}=$ $i \Gamma_{W} M_{W}, D_{12}^{\gamma}=s_{12}$, and we can replace $D_{12}^{Z}$ by $s_{12}-M_{Z}^{2}$ since $s_{12} \geq 4 M_{W}^{2}$. The QCD amplitude is

$$
\begin{aligned}
G^{W^{-}}(-,-,-) & =(-1) \frac{K_{g} Q_{W}^{2} U_{65}^{*}}{D_{56}^{W}} \\
& \times\left[\frac{U_{14} \delta_{23}}{s_{23}}\left(\frac{F_{125634}}{s_{234}}+\frac{F_{341256}}{s_{123}}\right)\right. \\
& \left.+\frac{U_{32} \delta_{14}}{s_{14}}\left(\frac{F_{345612}}{s_{124}}+\frac{F_{123456}}{s_{134}}\right)\right],
\end{aligned}
$$

where

$$
K_{g}=i e^{2} g^{2}\left(\frac{\lambda_{a}}{2}\right)_{c_{3} c_{2}}\left(\frac{\lambda_{a}}{2}\right)_{c_{1} c_{4}}
$$

The explicit $(-1)$ in this formula represents the relative minus sign between the electroweak ('annihilation') and QCD ('scattering') diagrams due to Fermi-Dirac statistics.

The amplitudes for the charge conjugate process $V_{l}=W^{+}, V_{q}=W^{-}$can be obtained directly from the Feynman rules, or by CP-conjugation from Eqs. (2.12) and (2.14) using the conventions described in Appendix A:

$$
\begin{aligned}
E^{W^{+} W^{-}}(-,-,-)= & \frac{K_{e} Q_{W}^{2} U_{43}^{*} U_{56} \delta_{12}}{D_{34}^{W} D_{56}^{W}} \\
& \times\left[-\frac{\left(1+\tau_{1}\right) Q_{W}^{2}}{2 s_{134}} F_{123456}-\frac{\left(1-\tau_{1}\right) Q_{W}^{2}}{2 s_{234}} F_{125634}\right. \\
& \left.+\left(\frac{L_{1} \cot \theta_{W}}{D_{12}^{Z}}+\frac{Q_{1}}{D_{12}^{\gamma}}\right)\left(F_{125634}-F_{123456}\right)\right] .
\end{aligned}
$$

We note that Eq. (2.16) can be obtained from Eq. (2.12) either by the formal interchange of labels $(3 \leftrightarrow 5,4 \leftrightarrow 6)$, or by the replacements $\tau_{1} \rightarrow-\tau_{1}, U \rightarrow U^{\dagger}$ which interchange the couplings of $W^{+}$and $W^{-}$to fermions, and by changing the sign of the $\left(L_{1} \cot \theta_{W} / D_{12}^{Z}+\right.$ $\left.Q_{1} / D_{12}^{\gamma}\right)$ term, which comes from the three-boson vertex. Similarly,

$$
\begin{aligned}
G^{W^{+}}(-,-,-)= & (-1) \frac{K_{g} Q_{W}^{2} U_{56}}{D_{56}^{W}} \\
& \times\left[\frac{U_{41}^{*} \delta_{23}}{s_{23}}\left(\frac{F_{125634}}{s_{234}}+\frac{F_{341256}}{s_{123}}\right)\right. \\
& \left.+\frac{U_{23}^{*} \delta_{14}}{s_{14}}\left(\frac{F_{345612}}{s_{124}}+\frac{F_{123456}}{s_{134}}\right)\right],
\end{aligned}
$$

which can be obtained from Eq. (2.14) simply by the replacement $U \rightarrow U^{\dagger}$. 


\section{Amplitudes for $V_{l}=W^{\mp}$, and $V_{q}=Z^{0}$}

In this case, the non-zero electroweak helicity amplitudes are $E(-, \mp,-)$. The QCD amplitudes are the same as in the preceding subsection, and once again, only $E(-,-,-)$ and $G(-,-,-)$ will contribute to chromo-electroweak interference.

$$
\begin{aligned}
E^{W^{-} Z}(-,-,-)= & \frac{K_{e} Q_{W}^{2} U_{12} U_{65}^{*} \delta_{34} L_{3}}{D_{34}^{Z} D_{56}^{W}} \\
& \times\left[\left(\frac{\cot \theta_{W}}{D_{12}^{W}}-\frac{L_{2}}{s_{234}}\right) F_{125634}\right. \\
& \left.-\left(\frac{\cot \theta_{W}}{D_{12}^{W}}+\frac{L_{1}}{s_{134}}\right) F_{123456}\right] .
\end{aligned}
$$

In this case, $f_{3}=u, d$ are both allowed while $f_{1}=d$. For the kinematic configurations of interest, $D_{34}^{Z}=i \Gamma_{Z} M_{Z}, D_{56}^{W}=i \Gamma_{W} M_{W}$, and $D_{12}^{W}=s_{12}-M_{W}^{2}$. The QCD amplitude $G^{W^{-}}$ is given by Eq. (2.14) with the flavor indices taking values appropriate to this process.

The electroweak amplitude for the charge conjugate process is

$$
\begin{aligned}
E^{W^{+} Z}(-,-,-)= & \frac{K_{e} Q_{W}^{2} U_{21}^{*} U_{56} \delta_{34} L_{3}}{D_{34}^{Z} D_{56}^{W}} \\
& \times\left[\left(\frac{\cot \theta_{W}}{D_{12}^{W}}-\frac{L_{1}}{s_{134}}\right) F_{123456}\right. \\
& \left.-\left(\frac{\cot \theta_{W}}{D_{12}^{W}}+\frac{L_{2}}{s_{234}}\right) F_{125634}\right],
\end{aligned}
$$

which can be obtained from Eq. (2.18) by the replacements $\cot \theta_{W} \rightarrow-\cot \theta_{W}$ and $U \rightarrow U^{\dagger}$. We note that the expressions in square brackets in Eqs. (2.18) and (2.19) can be expressed by the single formula

$$
\left[-\left(\frac{\tau_{1} \cot \theta_{W}}{D_{12}^{W}}+\frac{L_{1}}{s_{134}}\right) F_{123456}-\left(\frac{\tau_{2} \cot \theta_{W}}{D_{12}^{W}}+\frac{L_{2}}{s_{234}}\right) F_{125634}\right] .
$$

$G^{W^{+}}$is given by Eq. (2.17).

\section{Amplitudes for $V_{l}=Z^{0}$ and $V_{q}=W^{ \pm}$}

The non-zero electroweak helicity amplitudes are $E_{1-3}(-,-, \mp)$. Thus while all helicity combinations are allowed in the QCD amplitudes, only $G_{1-4}(-,-, \mp)$ will contribute to chromo-electroweak interference.

$$
\begin{aligned}
E^{Z W^{+}}(-,-,-)= & \frac{K_{e} Q_{W}^{2} U_{21}^{*} U_{34} \delta_{56} L_{5}}{D_{34}^{W} D_{56}^{Z}} \\
& \times\left[\left(\frac{\cot \theta_{W}}{D_{12}^{W}}-\frac{L_{1}}{s_{234}}\right) F_{125634}\right. \\
& \left.-\left(\frac{\cot \theta_{W}}{D_{12}^{W}}+\frac{L_{2}}{s_{134}}\right) F_{123456}\right] .
\end{aligned}
$$


Note that this amplitude can be obtained from Eq. (2.19) by formal interchange of labels $(3 \leftrightarrow 5,4 \leftrightarrow 6)$. The amplitude for production of a right-handed lepton pair is

$$
\begin{aligned}
E^{Z W^{+}}(-,-,+)= & \frac{K_{e} Q_{W}^{2} U_{21}^{*} U_{34} \delta_{56} R_{5}}{D_{34}^{W} D_{56}^{Z}} \\
& \times\left[\left(\frac{\cot \theta_{W}}{D_{12}^{W}}-\frac{L_{1}}{s_{234}}\right) F_{126534}\right. \\
& \left.-\left(\frac{\cot \theta_{W}}{D_{12}^{W}}+\frac{L_{2}}{s_{134}}\right) F_{123465}\right],
\end{aligned}
$$

which may be obtained from Eq. (2.21) by replacing $L_{5}$ by $R_{5}$ and interchanging labels $(5 \leftrightarrow 6)$. The required QCD amplitudes are

$$
\begin{aligned}
G^{Z}(-,-,-)= & -1) \frac{K_{g} \delta_{14} \delta_{23} \delta_{56} L_{5}}{D_{56}^{W}} \\
& \times\left[\frac{L_{1}}{s_{23}}\left(\frac{F_{125634}}{s_{234}}+\frac{F_{341256}}{s_{123}}\right)\right. \\
& \left.+\frac{L_{3}}{s_{14}}\left(\frac{F_{345612}}{s_{124}}+\frac{F_{123456}}{s_{134}}\right)\right], \\
G^{Z}(-,-,+)= & (-1) \frac{K_{g} \delta_{14} \delta_{23} \delta_{56} R_{5}}{D_{56}^{W}} \\
& \times\left[\frac{L_{1}}{s_{23}}\left(\frac{F_{126534}}{s_{234}}+\frac{F_{341265}}{s_{123}}\right)\right. \\
& \left.+\frac{L_{3}}{s_{14}}\left(\frac{F_{346512}}{s_{124}}+\frac{F_{123465}}{s_{134}}\right)\right] .
\end{aligned}
$$

The electroweak amplitudes for the charge conjugate processes are

$$
\begin{aligned}
E^{Z W^{-}}(-,-,-)= & \left.\frac{K_{e} Q_{W}^{2} U_{12} U_{43}^{*} \delta_{56} L_{5}}{D_{34}^{W} D_{56}^{Z}}\right] \\
& \times\left[-\left(\frac{\cot \theta_{W}}{D_{12}^{W}}+\frac{L_{1}}{s_{234}}\right) F_{125634}\right. \\
& \left.+\left(\frac{\cot \theta_{W}}{D_{12}^{W}}-\frac{L_{2}}{s_{134}}\right) F_{123456}\right],
\end{aligned}
$$

which can be got either from Eq. (2.18) by formally interchanging labels $(3 \leftrightarrow 5,4 \leftrightarrow 6)$, or from Eq. (2.21) by letting $\cot \theta_{W} \rightarrow-\cot \theta_{W}$ and $U \rightarrow U^{\dagger}$; and

$$
\begin{aligned}
E^{Z W^{-}}(-,-,+)= & \frac{K_{e} Q_{W}^{2} U_{12} U_{43}^{*} \delta_{56} R_{5}}{D_{34}^{W} D_{56}^{Z}} \\
& \times\left[-\left(\frac{\cot \theta_{W}}{D_{12}^{W}}+\frac{L_{1}}{s_{234}}\right) F_{126534}\right. \\
& \left.+\left(\frac{\cot \theta_{W}}{D_{12}^{W}}-\frac{L_{2}}{s_{134}}\right) F_{123465}\right] .
\end{aligned}
$$


We note that the expressions in square brackets in Eqs. (2.21) and (2.26) can be expressed by the single formula

$$
\left[-\left(\frac{\tau_{1} \cot \theta_{W}}{D_{12}^{W}}+\frac{L_{1}}{s_{234}}\right) F_{125634}-\left(\frac{\tau_{2} \cot \theta_{W}}{D_{12}^{W}}+\frac{L_{2}}{s_{134}}\right) F_{123456}\right] .
$$

The QCD amplitudes for the charge conjugate processes are given by Eqs. (2.23) and (2.24) with the flavor labels appropriately interpreted.

\section{Amplitudes for $V_{l}=Z^{0}$ and $V_{q}=Z^{0}$}

In $Z^{0}$ pair production, all helicity combinations will in general yield non-zero amplitudes. However, in the chromo-electroweak interference terms, the helicities of the initial- and final-state quarks are constrained to be the same, and we will only present results for these amplitudes.

$$
\begin{aligned}
& E^{Z Z}(-,-,-)=\frac{K_{e} \delta_{12} \delta_{34} \delta_{56} L_{1}^{2} L_{3} L_{5}}{D_{34}^{Z} D_{56}^{Z}}\left(-\frac{F_{125634}}{s_{234}}-\frac{F_{123456}}{s_{134}}\right), \\
& E^{Z Z}(-,-,+)=\frac{K_{e} \delta_{12} \delta_{34} \delta_{56} L_{1}^{2} L_{3} R_{5}}{D_{34}^{Z} D_{56}^{Z}}\left(-\frac{F_{126534}}{s_{234}}-\frac{F_{123465}}{s_{134}}\right), \\
& E^{Z Z}(+,+,+)=\frac{K_{e} \delta_{12} \delta_{34} \delta_{56} R_{1}^{2} R_{3} R_{5}}{D_{34}^{Z} D_{56}^{Z}}\left(\frac{F_{214365}}{s_{234}}+\frac{F_{216543}}{s_{134}}\right), \\
& E^{Z Z}(+,+,-)=\frac{K_{e} \delta_{12} \delta_{34} \delta_{56} R_{1}^{2} R_{3} L_{5}}{D_{34}^{Z} D_{56}^{Z}}\left(\frac{F_{214356}}{s_{234}}+\frac{F_{215643}}{s_{134}}\right) .
\end{aligned}
$$

Note that Eq. (2.30) can be obtained from Eq. (2.28) by changing left-handed $Z^{0}$ couplings to right, interchanging labels $(1 \leftrightarrow 2,3 \leftrightarrow 4,5 \leftrightarrow 6)$, and appending a minus sign.

The QCD amplitudes for $Z^{0}$ production with negative quark helicities were given in Eqs. (2.23) and (2.24). In addition, we need

$$
\begin{aligned}
G^{Z}(+,+,+)= & (-1) \frac{K_{g} \delta_{14} \delta_{23} \delta_{56} R_{5}}{D_{56}^{W}} \\
& \times\left[-\frac{R_{1}}{s_{23}}\left(\frac{F_{214365}}{s_{234}}+\frac{F_{436521}}{s_{123}}\right)\right. \\
& \left.-\frac{R_{3}}{s_{14}}\left(\frac{F_{432165}}{s_{124}}+\frac{F_{216543}}{s_{134}}\right)\right],
\end{aligned}
$$

\footnotetext{
${ }^{2}$ This minus sign is omitted in Eq. (16) of [15]. The relative phase of amplitudes with different helicities is of course arbitrary: the negative sign in this case is consistent with a naive application of the Feynman rules, and with the conventions for charge conjugation discussed in Appendix A; it arises from charge conjugating the virtual quark in Fig. 5 .
} 


$$
\begin{aligned}
G^{Z}(+,+,-)= & (-1) \frac{K_{g} \delta_{14} \delta_{23} \delta_{56} L_{5}}{D_{56}^{W}} \\
& \times\left[-\frac{R_{1}}{s_{23}}\left(\frac{F_{214356}}{s_{234}}+\frac{F_{435621}}{s_{123}}\right)\right. \\
& \left.-\frac{R_{3}}{s_{14}}\left(\frac{F_{432156}}{s_{124}}+\frac{F_{215643}}{s_{134}}\right)\right] .
\end{aligned}
$$

These two amplitudes may be obtained from Eqs. (2.23) and (2.24) by changing left- to right-handed $Z$ couplings, interchanging labels $(1 \leftrightarrow 2,3 \leftrightarrow 4,5 \leftrightarrow 6)$, and appending a minus sign. The interchange of labels $(1 \leftrightarrow 4,3 \leftrightarrow 2,5 \leftrightarrow 6)$ with the change of couplings and an overall minus sign also produces the desired result.

\section{Amplitudes for $V_{l}=\gamma$ and $V_{q}=W^{ \pm}, Z^{0}$}

The amplitudes for production of a photon (either real or virtual) plus two jets can in principle be obtained from the corresponding amplitudes for production of a $Z^{0}$ plus two jets by setting $M_{Z}=0$ and $R_{5}=L_{5}=1$. In the case of a real photon, the limit $M_{Z} \rightarrow 0$ is rather subtle since the photon has zero width, and it is simpler to derive the amplitudes from scratch. The amplitude for production of a photon that is off-shell by an amount $Q^{2}$ is suppressed relative that for an on-shell $Z^{0}$ by a factor of $\Gamma_{Z} M_{Z} / Q^{2}$ : thus, photons with $Q^{2} \geq 10 \mathrm{GeV}$ are not likely to be phenomenologically very interesting, and we will therefore confine our attention to real photons. Following [15] we present the amplitudes in terms of the functions 3

$$
\begin{aligned}
& H_{12345}=2 \sqrt{2} \frac{\langle 1-\mid 3+\rangle^{2}\langle 3+\mid 4-\rangle}{\langle 1-\mid 5+\rangle\langle 2-\mid 5+\rangle}, \\
& H_{12345}^{+}=2 \sqrt{2} \frac{\langle 3+\mid 1-\rangle^{2}\langle 4-\mid 3+\rangle}{\langle 5+\mid 1-\rangle\langle 5+\mid 2-\rangle},
\end{aligned}
$$

of the outgoing momenta $k_{i}, i=1, \ldots, 5$, which satisfy momentum conservation $\sum_{i=1}^{5} k_{i}=0$. Here, $k_{5}$ is the momentum of the real photon (compare Eq. (2.9) et seq.). Amplitudes will be labeled by $\left(\operatorname{sign}\left(\lambda_{1}\right), \operatorname{sign}\left(\lambda_{3}\right), \operatorname{sign}\left(\lambda_{5}\right)\right)$, where $\lambda_{5}$ is now the helicity of the photon $\left(\lambda_{5}= \pm 1\right)$.

For $V_{q}=W^{+}$, the non-zero electroweak helicity amplitudes are

$$
E^{\gamma W^{+}}(-,-,+)=\frac{K_{e}^{\gamma} Q_{W}^{2} U_{21}^{*} U_{34}}{D_{34}^{W}}\left(Q_{2}-\tau_{1} \frac{s_{25}}{D_{12}^{W}}\right) H_{12345}
$$

and

$$
E^{\gamma W^{+}}(-,-,-)=\frac{K_{e}^{\gamma} Q_{W}^{2} U_{21}^{*} U_{34}}{D_{34}^{W}}\left(Q_{1}-\tau_{2} \frac{s_{15}}{D_{12}^{W}}\right) H_{21435}^{+}
$$

\footnotetext{
${ }^{3}$ We note that $H_{12345}=-C(1,2,3,4,5)=-(\langle 1-\mid 3+\rangle)^{2} \delta$ where $C$ and $\delta$ are defined in Eqs. $(19,20)$ of [15]. $H^{+}=H^{*}$ if $k_{i}^{0}>0, i=1, \ldots, 5$, see Appendix A.
} 
where

$$
K_{e}^{\gamma} \equiv i e^{3} \delta_{c_{1} c_{2}} \delta_{c_{3} c_{4}} .
$$

We note that for a weak-isospin doublet $\Delta Q=\Delta \tau / 2$ and $Q_{1}-Q_{2}=\tau_{1}=-\tau_{2}$, so that when the $W$ is on shell, i.e., when $s_{34}=M_{W}^{2}$,

$$
Q_{2}-\tau_{1} \frac{s_{25}}{s_{12}-M_{W}^{2}}=Q_{1}-\tau_{2} \frac{s_{15}}{s_{12}-M_{W}^{2}} .
$$

The non-zero electroweak amplitudes for the case $V_{q}=W^{-}$are

$$
E^{\gamma W^{-}}(-,-,+)=\frac{K_{e}^{\gamma} Q_{W}^{2} U_{12} U_{43}^{*}}{D_{34}^{W}}\left(Q_{2}-\tau_{1} \frac{s_{25}}{D_{12}^{W}}\right) H_{12345},
$$

and

$$
E^{\gamma W^{-}}(-,-,-)=\frac{K_{e}^{\gamma} Q_{W}^{2} U_{12} U_{43}^{*}}{D_{34}^{W}}\left(Q_{1}-\tau_{2} \frac{s_{15}}{D_{12}^{W}}\right) H_{21435}^{+},
$$

which are identical in form to Eqs. (2.35) and (2.36) except that $U$ is replaced by $U^{\dagger}$.

For the case that $V_{q}=Z^{0}$, all helicity combinations yield non-vanishing electroweak amplitudes. However, only amplitudes with the same helicities for the quarks (i.e., $\lambda_{1}=\lambda_{3}$ ) contribute to chromo-electroweak interference. These amplitudes are

$$
\begin{aligned}
& E^{\gamma Z}(+,+,+)=\frac{K_{e}^{\gamma} \delta_{12} \delta_{34} Q_{1}}{D_{34}^{Z}} R_{1} R_{3} H_{21435}, \\
& E^{\gamma Z}(+,+,-)=\frac{K_{e}^{\gamma} \delta_{12} \delta_{34} Q_{1}}{D_{34}^{Z}} R_{1} R_{3} H_{12345}^{+}, \\
& E^{\gamma Z}(-,-,+)=\frac{K_{e}^{\gamma} \delta_{12} \delta_{34} Q_{1}}{D_{34}^{Z}} L_{1} L_{3} H_{12345}, \\
& E^{\gamma Z}(-,-,-)=\frac{K_{e}^{\gamma} \delta_{12} \delta_{34} Q_{1}}{D_{34}^{Z}} L_{1} L_{3} H_{21435}^{+} .
\end{aligned}
$$

The QCD amplitudes that can interfere with the electroweak amplitudes given in Eqs. (2.35) through (2.44) are

$$
\begin{aligned}
& G^{\gamma}(+,+,+)=(-1) K_{g}^{\gamma} \delta_{14} \delta_{23}\left(-\frac{Q_{1}}{s_{23}} H_{41235}-\frac{Q_{3}}{s_{14}} H_{23415}\right), \\
& G^{\gamma}(+,+,-)=(-1) K_{g}^{\gamma} \delta_{14} \delta_{23}\left(\frac{Q_{1}}{s_{23}} H_{14325}^{+}+\frac{Q_{3}}{s_{14}} H_{32145}^{+}\right), \\
& G^{\gamma}(-,-,+)=(-1) K_{g}^{\gamma} \delta_{14} \delta_{23}\left(\frac{Q_{1}}{s_{23}} H_{14325}+\frac{Q_{3}}{s_{14}} H_{32145}\right), \\
& G^{\gamma}(-,-,-)=(-1) K_{g}^{\gamma} \delta_{14} \delta_{23}\left(-\frac{Q_{1}}{s_{23}} H_{41235}^{+}-\frac{Q_{3}}{s_{14}} H_{23415}^{+}\right),
\end{aligned}
$$

where

$$
K_{g}^{\gamma} \equiv i e g^{2}\left(\frac{\lambda_{a}}{2}\right)_{c_{3} c_{2}}\left(\frac{\lambda_{a}}{2}\right)_{c_{1} c_{4}} .
$$




\section{Squared Amplitudes: Chromo-electroweak Interference Terms}

The amplitudes presented in the preceding section can easily be evaluated numerically and the resulting complex numbers squared to obtain a cross section. We will, however, square the helicity amplitudes analytically and present formulas for the chromo-electroweak interference contributions to the cross section. These formulas turn out to be as simple and compact as the helicity amplitudes themselves, and their analytic forms explicitly exhibit their parity-violating nature and are therefore quite instructive.

The interference contributions will all be linear functions of the invariants

$$
x_{i j l m} \equiv \epsilon_{\mu \nu \lambda \sigma} k_{i}^{\mu} k_{j}^{\nu} k_{l}^{\lambda} k_{m}^{\sigma}
$$

where $\epsilon_{\mu \nu \lambda \sigma}$ is the totally antisymmetic Levi-Civita tensor with $\epsilon_{0123}=+1$, and $k_{l}$ are fourmomenta. In processes of the type $q_{1} \bar{q}_{2} \rightarrow\left(V_{q} \rightarrow q_{3} \bar{q}_{4}\right)+V_{l}$, where $V_{l}$ is detected as a real particle, there are only four independent momenta and there is therefore essentially only one parity-odd invariant $x_{1234}$. In this case, the use of traditional trace-algebra techniques to compute spin-averaged squared matrix elements leads to essentially the same analytic forms as does use of the helicity amplitude methods. However, if $V_{l} \rightarrow l_{5} \bar{l}_{6}$, there are five independent momenta and hence five essentially different parity-odd invariants (e.g., $\left.x_{1234}, x_{1235}, x_{1245}, x_{1345}, x_{2345}\right)$. These five invariants are not functionally independent however: for a process involving $n$ particles, the number of functionally independent invariants is $3 n-10$, and this number includes the scalar products $k_{i} \cdot k_{j}$. F For a $2 \rightarrow 4$ process, only 8 of the 15 invariants $s_{i j}$ and $x_{i j l m}$ are functionally independent, and the relations between them are nonlinear and not trivial. In this case, there is a dramatic difference between the use of trace-algebra and helicity-amplitude techniques: straightforward application of the former leads to interference contributions that involve in excess of a hundred terms linear in the invariants $x_{i j l m}$, while squaring the helicity amplitudes generates on the order of ten such terms! In addition, it is very difficult to show analytically that the expressions obtained using the two methods are in fact identical (though their equality can be checked numerically). The calculation of the 4-particle interference contributions thus provides a rather striking demonstration of the advantages of using helicity amplitude techniques.

\section{Four particle final states: $V_{q} \rightarrow q_{3} \bar{q}_{4}, V_{l} \rightarrow l_{5} \bar{l}_{6}$}

In this section, we will present results for the chromo-electroweak interference contributions $\mathcal{J}_{4}$ to the helicity- and color-averaged squared amplitudes $(E+G)\left(E^{+}+G^{+}\right)$for fixed flavors, i.e.,

\footnotetext{
${ }^{4}$ There is in addition one discrete invariant which can take two values and which describes the handedness of the coordinate system used.

${ }^{5}$ This is because $x_{i j l m}= \pm\left[-G\left(\begin{array}{l}k_{i} k_{j} k_{l} k_{m} \\ k_{i} k_{j} k_{l} k_{m}\end{array}\right)\right]^{1 / 2}$ where $G$ is a Gram determinant (see e.g., [16]). Thus, linear relations between the different $x$ 's have coefficients that are cubic in the invariants $s_{i j}$.
} 


$$
\mathcal{J}_{4}^{V_{l} V_{q}} \equiv \frac{1}{4 n_{c}^{2}} \sum_{\text {colors helicities }} \sum_{\operatorname{Re}}\left[E^{V_{l} V_{q}}\left(G^{V_{l} V_{q}}\right)^{+}+\left(E^{V_{l} V_{q}}\right)^{+} G^{V_{l} V_{q}}\right]
$$

The operation denoted by the superscript + is the crossing invariant extension of complex conjugation of amplitudes involving particles with negative as well as physical energies as explained in Appendix A. These contributions are most compactly expressed in terms of a set of auxiliary functions of the external momenta which we define as follows:

$$
\begin{gathered}
I_{123456} \equiv F_{123456} F_{123456}^{+}, \\
J_{123456} \equiv F_{123456} F_{125634}^{+}, \\
K_{123456} \equiv F_{123456} F_{341256}^{+}, \\
X_{123456} \equiv \frac{1}{s_{14}}\left(\frac{I_{123456}}{s_{134}}+\frac{J_{321456}}{s_{124}}\right), \\
Y_{123456} \equiv \frac{1}{s_{23}}\left(\frac{J_{123456}}{s_{234}}+\frac{K_{123456}}{s_{123}}\right) .
\end{gathered}
$$

In fact, only the imaginary parts of the functions $X$ and $Y$ will contribute to the interference terms. This can be seen, for example, by writing out the squared matrix element explicitly in the case of $W^{+} W^{-}$production using Eqs. (2.12) and (2.14):

$$
\begin{aligned}
& E^{W^{-}} W^{+}(-,-,-)\left[G^{W^{-}}(-,-,-)\right]^{+}=(-1) \frac{K_{e} K_{g}^{*} Q_{W}^{4}\left|U_{34}\right|^{2}\left|U_{65}\right|^{2} \delta_{12}}{D_{34}^{W}\left|D_{56}^{W}\right|^{2}} \\
& \times\left[\left(\frac{L_{1} \cot \theta_{W}}{s_{12}-M_{Z}^{2}}+\frac{Q_{1}}{s_{12}}+\frac{\left(\tau_{1}-1\right) Q_{W}^{2}}{2 s_{134}}\right) F_{123456}\right. \\
&\left.-\left(\frac{L_{1} \cot \theta_{W}}{s_{12}-M_{Z}^{2}}+\frac{Q_{1}}{s_{12}}+\frac{\left(\tau_{1}+1\right) Q_{W}^{2}}{2 s_{234}}\right) F_{125634}\right] \\
& \times\left[\frac{\delta_{23}}{s_{23}}\left(\frac{F_{125634}^{+}}{s_{234}}+\frac{F_{341256}^{+}}{s_{123}}\right)+\frac{\delta_{14}}{s_{14}}\left(\frac{F_{345612}^{+}}{s_{124}}+\frac{F_{123456}^{+}}{s_{134}}\right)\right] \\
&=\frac{K_{e} K_{g}^{*} Q_{W}^{4}\left|U_{34}\right|^{2}\left|U_{65}\right|^{2} \delta_{12}}{D_{34}^{W}\left|D_{56}^{W}\right|^{2}} \\
& \quad \times\left[\left(\frac{L_{1} \cot \theta_{W}}{s_{12}-M_{Z}^{2}}+\frac{Q_{1}}{s_{12}}+\frac{\left(\tau_{1}+1\right) Q_{W}^{2}}{2 s_{234}}\right)\left(\delta_{23} X_{214365}^{+}+\delta_{14} Y_{214365}^{+}\right)\right. \\
&\left.-\left(\frac{L_{1} \cot \theta_{W}}{s_{12}-M_{Z}^{2}}+\frac{Q_{1}}{s_{12}}+\frac{\left(\tau_{1}-1\right) Q_{W}^{2}}{2 s_{134}}\right)\left(\delta_{23} Y_{123456}+\delta_{14} X_{123456}\right)\right] .
\end{aligned}
$$

This rather compact form was obtained by using the symmetry properties of the functions $I, J$ and $K$ discussed in Appendix B-see Eqs. (B7, B9, B15). We now note that $D_{12}^{W}=$ $s_{34}-M_{W}^{2}+i \Gamma_{W} M_{W}$ is imaginary on shell, that is, when $s_{34}=M_{W}^{2}$. In [8], we integrated the 
cross section over a small interval $\left(M_{W}-\Delta\right)^{2} \leq s_{34} \leq\left(M_{W}+\Delta\right)^{2}$, with $\Delta \simeq \Gamma_{W}$. However, in this paper, we will use the narrow width approximation and replace

$$
\frac{1}{D_{34}^{W}} \longrightarrow-i \pi \delta\left(s_{34}-M_{W}^{2}\right)
$$

The two procedures yield roughly the same numerical results. The narrow width approximation is, at least theoretically if not also numerically, the more consistent of the two procedures since the diagrams we include are gauge invariant only when $V_{q}\left(W^{+}\right.$in this case) is exactly on shell. We also use a narrow width approximation for $V_{l}\left(W^{+}\right.$in this case)

$$
\frac{1}{\left|D_{56}^{W}\right|^{2}} \longrightarrow \frac{\pi}{\Gamma_{W} M_{W}} \delta\left(s_{56}-M_{w}^{2}\right) \text {. }
$$

Performing the color and helicity final state sums and initial state averages, we obtain

$$
\begin{aligned}
& \mathcal{J}_{4}^{W^{-} W^{+}}=\mathcal{K}_{4}^{W W} Q_{W}^{4} \delta_{12}\left|U_{34}\right|^{2}\left|U_{65}\right|^{2} \\
& \times\left[\left(\frac{L_{1} \cot \theta_{W}}{s_{12}-M_{Z}^{2}}+\frac{Q_{1}}{s_{12}}+\frac{\left(\tau_{1}+1\right) Q_{W}^{2}}{2 s_{234}}\right)\left(\delta_{23} \operatorname{Im} X_{214365}^{+}+\delta_{14} \operatorname{Im} Y_{214365}^{+}\right)\right. \\
& \left.\quad-\left(\frac{L_{1} \cot \theta_{W}}{s_{12}-M_{Z}^{2}}+\frac{Q_{1}}{s_{12}}+\frac{\left(\tau_{1}-1\right) Q_{W}^{2}}{2 s_{134}}\right)\left(\delta_{23} \operatorname{Im} Y_{123456}+\delta_{14} \operatorname{Im} X_{123456}\right)\right],
\end{aligned}
$$

where,

$$
\mathcal{K}_{4}^{V_{l} V_{q}}=\frac{2^{7} \pi^{6} \alpha^{3} \alpha_{s} C_{F}}{n_{c}} \frac{\delta\left(s_{34}-M_{V_{q}}^{2}\right) \delta\left(s_{56}-M_{V_{l}}^{2}\right)}{\Gamma_{V_{l}} M_{V_{l}}}
$$

Explicit formulas for the imaginary parts of the functions $X$ and $Y$ are derived in Appendix B:

$$
\begin{aligned}
\operatorname{Im} X_{123456}= & 32 \frac{s_{135}}{s_{14} s_{124}}\left[\left(s_{15}+s_{35}\right) x_{1234}+s_{13} x_{1245}+s_{24} x_{1345}\right] \\
\operatorname{Im} Y_{123456}= & 32 \frac{s_{135}}{s_{23}}\left[\frac{s_{13}}{s_{123}}\left(x_{1245}-x_{2345}\right)\right. \\
& \left.-\frac{1}{s_{234}}\left\{\left(s_{15}+s_{35}\right) x_{1234}+s_{13} x_{2345}+s_{24} x_{1345}\right\}\right] .
\end{aligned}
$$

For the charge conjugate process, that is for $V_{q}=W^{-} \rightarrow d \bar{u}, V_{l}=W^{+} \rightarrow \nu \bar{l}$,

$$
\begin{aligned}
& \mathcal{J}_{4}^{W^{+} W^{-}}=\mathcal{K}_{4}^{W W} Q_{W}^{4} \delta_{12}\left|U_{43}\right|^{2}\left|U_{56}\right|^{2} \\
& \quad \times\left[\left(\frac{L_{1} \cot \theta_{W}}{s_{12}-M_{Z}^{2}}+\frac{Q_{1}}{s_{12}}+\frac{\left(\tau_{1}+1\right) Q_{W}^{2}}{2 s_{134}}\right)\left(\delta_{14} \operatorname{Im} X_{123456}+\delta_{23} \operatorname{Im} Y_{123456}\right)\right. \\
& \left.\quad-\left(\frac{L_{1} \cot \theta_{W}}{s_{12}-M_{Z}^{2}}+\frac{Q_{1}}{s_{12}}+\frac{\left(\tau_{1}-1\right) Q_{W}^{2}}{2 s_{234}}\right)\left(\delta_{14} \operatorname{Im} Y_{214365}^{+}+\delta_{23} \operatorname{Im} X_{214365}^{+}\right)\right]
\end{aligned}
$$

Note that Eq. (2.60) and Eq. (2.64) are related by the interchanges $(1 \leftrightarrow 2,3 \leftrightarrow 4,5 \leftrightarrow$ $6, X \leftrightarrow X^{+}, Y \leftrightarrow Y^{+}$), or alternatively by the replacements $U \rightarrow U^{\dagger}, \tau_{1} \rightarrow-\tau_{1}$, $\cot \theta_{W} \rightarrow$ $-\cot \theta_{W}$, and $Q_{1} \rightarrow-Q_{1}$. 
For the case $V_{q}=Z^{0} \rightarrow q_{3} \bar{q}_{4}, V_{l}=W^{-} \rightarrow l_{5} \bar{\nu}_{6}$,

$$
\begin{aligned}
& \mathcal{J}_{4}^{W^{-} Z}=\mathcal{K}_{4}^{W Z} Q_{W}^{4}\left|U_{12}\right|^{2} \delta_{34}\left|U_{65}\right|^{2} L_{3} \\
& \quad \times\left[\left(\frac{\tau_{1} \cot \theta_{W}}{s_{12}-M_{W}^{2}}+\frac{L_{1}}{s_{134}}\right)\left(\delta_{14} \operatorname{Im} X_{123456}+\delta_{23} \operatorname{Im} Y_{123456}\right)\right. \\
& \left.\quad+\left(\frac{\tau_{2} \cot \theta_{W}}{s_{12}-M_{W}^{2}}+\frac{L_{2}}{s_{234}}\right)\left(\delta_{14} \operatorname{Im} Y_{214365}^{+}+\delta_{23} \operatorname{Im} X_{214365}^{+}\right)\right],
\end{aligned}
$$

while for the charge conjugate process, that is for $V_{l}=W^{+} \rightarrow \nu_{5} \bar{l}_{6}$,

$$
\begin{aligned}
& \mathcal{J}_{4}^{W^{+} Z}=\mathcal{K}_{4}^{W Z} Q_{W}^{4}\left|U_{21}\right|^{2} \delta_{34}\left|U_{56}\right|^{2} L_{3} \\
& \times\left[\left(\frac{\tau_{1} \cot \theta_{W}}{s_{12}-M_{W}^{2}}+\frac{L_{1}}{s_{134}}\right)\left(\delta_{14} \operatorname{Im} X_{123456}+\delta_{23} \operatorname{Im} Y_{123456}\right)\right. \\
& \left.\quad+\left(\frac{\tau_{2} \cot \theta_{W}}{s_{12}-M_{W}^{2}}+\frac{L_{2}}{s_{234}}\right)\left(\delta_{14} \operatorname{Im} Y_{214365}^{+}+\delta_{23} \operatorname{Im} X_{214365}^{+}\right)\right] .
\end{aligned}
$$

Eqs. (2.65) and (2.66) are related by the interchanges $(1 \leftrightarrow 2,3 \leftrightarrow 4,5 \leftrightarrow 6, X \leftrightarrow$ $X^{+}, Y \leftrightarrow Y^{+}$), or alternatively by the replacement $U \rightarrow U^{\dagger}$.

For the case $V_{q}=W^{+} \rightarrow u_{3} \bar{d}_{4}, V_{l}=Z^{0} \rightarrow l_{5} \bar{l}_{6}$,

$$
\begin{aligned}
& \mathcal{J}_{4}^{Z W^{+}}=\mathcal{K}_{4}^{Z W} Q_{W}^{2}\left|U_{21}\right|^{2} \delta_{14} \delta_{23} \delta_{56} \\
& \times\left[L _ { 5 } ^ { 2 } \left\{\left(\frac{\tau_{2} \cot \theta_{W}}{s_{12}-M_{W}^{2}}+\frac{L_{2}}{s_{134}}\right)\left(L_{2} \operatorname{Im} X_{123456}+L_{1} \operatorname{Im} Y_{123456}\right)\right.\right. \\
& \left.\quad+\left(\frac{\tau_{1} \cot \theta_{W}}{s_{12}-M_{W}^{2}}+\frac{L_{1}}{s_{234}}\right)\left(L_{2} \operatorname{Im} Y_{214365}^{+}+L_{1} \operatorname{Im} X_{214365}^{+}\right)\right\} \\
& \left.\quad+R_{5}^{2}\{5 \leftrightarrow 6\}\right],
\end{aligned}
$$

while for the charge conjugate process,

$$
\begin{aligned}
& \mathcal{J}_{4}^{Z W^{-}}=\mathcal{K}_{4}^{Z W} Q_{W}^{2}\left|U_{12}\right|^{2} \delta_{14} \delta_{23} \delta_{56} \\
& \times\left[L _ { 5 } ^ { 2 } \left\{\left(\frac{\tau_{1} \cot \theta_{W}}{s_{12}-M_{W}^{2}}+\frac{L_{1}}{s_{234}}\right)\left(L_{2} \operatorname{Im} Y_{214365}^{+}+L_{1} \operatorname{Im} X_{214365}^{+}\right)\right.\right. \\
& \left.\quad+\left(\frac{\tau_{2} \cot \theta_{W}}{s_{12}-M_{W}^{2}}+\frac{L_{2}}{s_{134}}\right)\left(L_{2} \operatorname{Im} X_{123456}+L_{1} \operatorname{Im} Y_{123456}\right)\right\} \\
& \left.\quad+R_{5}^{2}\{5 \leftrightarrow 6\}\right],
\end{aligned}
$$

which can be obtained from Eq. (2.66) by the interchanges $(1 \leftrightarrow 2,3 \leftrightarrow 4,5 \leftrightarrow 6, X \leftrightarrow$ $X^{+}, Y \leftrightarrow Y^{+}$), or by letting $U \rightarrow U^{\dagger}$. The two expressions are in fact formally identical, but they are not equal because the flavors involved are different in the two cases.

Finally, for the case $V_{q}=Z^{0} \rightarrow q_{3} \bar{q}_{4}, V_{l}=Z^{0} \rightarrow l_{5} \bar{l}_{6}$,

$$
\mathcal{J}_{4}^{Z Z}=\mathcal{K}_{4}^{Z Z} \delta_{12} \delta_{14} \delta_{23}\left[\left(L_{1}^{4} L_{5}^{2}-R_{1}^{4} R_{5}^{2}\right)\right.
$$




$$
\begin{aligned}
& \times\left\{\frac{1}{s_{134}}\left(\operatorname{Im} X_{123456}+\operatorname{Im} Y_{123456}\right)+\frac{1}{s_{234}}\left(\operatorname{Im} X_{214365}^{+}+\operatorname{Im} Y_{214365}^{+}\right)\right\} \\
& \left.+\left(L_{1}^{4} R_{5}^{2}-R_{1}^{4} L_{5}^{2}\right)\{5 \leftrightarrow 6\}\right] .
\end{aligned}
$$

\section{Three particle final states $\left(V_{q} \rightarrow q_{3} \bar{q}_{4}\right)+\left(V_{l}=W^{ \pm}\right.$or $\left.Z^{0}\right)$}

In the preceding subsection we presented chromo-electroweak interference contributions to the cross section for the production of two electroweak bosons, one of which $V_{q}$ decays to a quark-antiquark pair, and the other $V_{l}$ to a lepton pair, and in which we used the narrow width approximation for the propagators of both bosons. In this subsection we will consider production of a real $V_{l}$. If $V_{l}$ is a real photon, it can be detected experimentally; in the cases $V_{l}=W^{ \pm}, Z^{0}$, which might be detected for example through particular leptonic decay channels, our cross sections must be multiplied by the appropriate branching ratio.

The case of a real $V_{l}=W^{ \pm}, Z^{0}$ can be treated by introducing helicity eigenfunctions for the massive vector boson and computing the required helicity amplitudes. It is simpler, however, to integrate the expressions for the chromo-electroweak interference cross sections given in the preceding subsection over the phase space of the final leptonic decay products with the momentum of $V_{l}$ held fixed, and divide the result by the leptonic branching ratio.

We wish to integrate the squared amplitude over the $k_{5} k_{6}$ phase space holding $k_{5}+k_{6}$ fixed. If the narrow width approximation

$$
\frac{1}{\left|D_{56}^{V_{l}}\right|^{2}}=\frac{\pi \delta\left(s_{56}-M_{V_{l}}^{2}\right)}{\Gamma_{V_{l}} M_{V_{l}}}
$$

is used for the $V_{l}$ propagator, the $k_{5} k_{6}$ invariant phase space can be converted to the appropriate phase space for a real $V_{l}$ as follows:

$$
\int \frac{d^{3} k_{5} d^{3} k_{6}}{(2 \pi)^{6} 4 k_{5}^{0} k_{6}^{0}} \frac{\delta^{4}\left(\sum_{i=1}^{6} k_{i}\right)}{\left|D_{56}^{V_{l}}\right|^{2}}=\frac{1}{16 \pi M_{V_{l}} \Gamma_{V_{l}}} \int \frac{d^{3} q}{(2 \pi)^{3} 2 q^{0}} \delta^{4}\left(q+\sum_{i=1}^{4} k_{i}\right) .
$$

The 4-particle squared amplitudes presented in the preceding subsection were for a particular decay channel $V_{l} \rightarrow l_{5} \bar{l}_{6}$. If the coupling in this channel is $-i e \gamma^{\mu}\left(\mathcal{L} \gamma_{L}+\mathcal{R} \gamma_{R}\right)$, the partial width

$$
\Gamma_{V_{l}}^{l \bar{l}}=\frac{e^{2}\left(|\mathcal{L}|^{2}+|\mathcal{R}|^{2}\right) M_{V_{l}}}{24 \pi}
$$

and we need to divide the 4-particle squared amplitudes by the branching ratio $\Gamma_{V_{l}}^{l \bar{l}} / \Gamma_{V_{l}}$. The 4 -particle squared amplitudes are polynomials in $k_{5}$ when $q=k_{5}+k_{6}$ is held fixed. The averages over the decay phase-space can be effected by using the formulas

$$
\begin{aligned}
\int_{5,6} & \equiv \int \frac{d^{3} k_{5} d^{3} k_{6}}{(2 \pi)^{6} 4 k_{5}^{0} k_{6}^{0}}(2 \pi)^{4} \delta^{4}\left(q-k_{5}-k_{6}\right) \\
& =\frac{1}{8 \pi}
\end{aligned}
$$




$$
\begin{aligned}
\int_{5,6} k_{5}^{\mu} & =\frac{1}{2} q^{\mu} \int_{5,6}, \\
\int_{5,6} k_{5}^{\mu} k_{5}^{\nu} & =\left(\frac{1}{3} q^{\mu} q^{\nu}-\frac{1}{12} g^{\mu \nu} q^{2}\right) \int_{5,6},
\end{aligned}
$$

etc. From Eqs. (2.71) through (2.73) it is easy to see that the 3-particle squared amplitudes can be obtained from the corresponding 4-particle squared amplitudes given in the preceding subsection by the replacements

$$
\begin{aligned}
& \frac{1}{\left|D_{56}^{V_{l}}\right|^{2}} \approx \frac{\pi \delta\left(s_{56}-M_{V_{l}}^{2}\right)}{\Gamma_{V_{l}} M_{V_{l}}} \quad \rightarrow\left(\frac{1}{16 \pi \Gamma_{V_{L}} M_{V_{l}}}\right)\left(\frac{\Gamma_{V_{l}}}{\Gamma_{V_{l}}^{l l}}\right) \\
& =\frac{3}{2 e^{2}\left(|\mathcal{L}|^{2}+|\mathcal{R}|^{2}\right)}, \\
& k_{5}^{\mu} \rightarrow \frac{1}{2} q^{\mu}, \\
& k_{5}^{\mu} k_{6}^{\nu} \rightarrow \frac{1}{3} q^{\mu} q^{\nu}-\frac{1}{12} g^{\mu \nu} q^{2} .
\end{aligned}
$$

In particular, Eqs. (2.73) require the following replacements for Eqs. (2.62,2.63):

$$
\begin{gathered}
\operatorname{Im} X_{123456} \rightarrow \frac{32}{3} M_{V_{l}}^{2} \frac{x_{1234}}{s_{14} s_{124}}\left(s_{12}+s_{34}+s_{14}+s_{23}\right), \\
\operatorname{Im} Y_{123456} \rightarrow-\frac{32}{3} M_{V_{l}}^{2} \frac{x_{1234}}{s_{23} s_{234}}\left(s_{12}+s_{34}+s_{14}+s_{23}\right) .
\end{gathered}
$$

Let us define in analogy with Eq. (2.51)

$$
\mathcal{J}_{3}^{V_{l} V_{q}} \equiv \frac{1}{4 n_{c}^{2}} \sum_{\text {colors helicities }} \sum_{\operatorname{Re}}\left[E^{V_{l} V_{q}}\left(G^{V_{l} V_{q}}\right)^{+}+\left(E^{V_{l} V_{q}}\right)^{+} G^{V_{l} V_{q}}\right] .
$$

We also define

$$
\mathcal{K}_{3}^{V_{q}} \equiv \frac{2^{9} \pi^{4} \alpha^{2} \alpha_{s} C_{F}}{n_{c}} \delta\left(s_{34}-M_{V_{q}}^{2}\right) .
$$

Then, from Eqs. (2.60)-(2.69) we obtain the 3-particle squared amplitudes listed below:

$$
\begin{gathered}
\mathcal{J}_{3}^{W^{-} W^{+}}=\mathcal{K}_{3}^{W} Q_{W}^{2} \delta_{12}\left|U_{34}\right|^{2}\left(s_{12}+s_{34}+s_{14}+s_{23}\right) x_{1234} \\
\times\left[\left(\frac{L_{1} \cot \theta_{W}}{s_{12}-M_{Z}^{2}}+\frac{Q_{1}}{s_{12}}+\frac{\left(\tau_{1}+1\right) Q_{W}^{2}}{2 s_{234}}\right)\left(\frac{\delta_{14}}{s_{14} s_{134}}-\frac{\delta_{23}}{s_{23} s_{123}}\right)\right. \\
\left.-\left(\frac{L_{1} \cot \theta_{W}}{s_{12}-M_{Z}^{2}}+\frac{Q_{1}}{s_{12}}+\frac{\left(\tau_{1}-1\right) Q_{W}^{2}}{2 s_{134}}\right)\left(\frac{\delta_{14}}{s_{14} s_{124}}-\frac{\delta_{23}}{s_{23} s_{234}}\right)\right], \\
\mathcal{J}_{3}^{W^{+} W^{-}}=\mathcal{K}_{3}^{W} Q_{W}^{2} \delta_{12}\left|U_{43}\right|^{2}\left(s_{12}+s_{34}+s_{14}+s_{23}\right) x_{1234} \\
\times\left[\left(\frac{L_{1} \cot \theta_{W}}{s_{12}-M_{Z}^{2}}+\frac{Q_{1}}{s_{12}}+\frac{\left(\tau_{1}+1\right) Q_{W}^{2}}{2 s_{134}}\right)\left(\frac{\delta_{14}}{s_{14} s_{124}}-\frac{\delta_{23}}{s_{23} s_{234}}\right)\right. \\
\left.\quad-\left(\frac{L_{1} \cot \theta_{W}}{s_{12}-M_{Z}^{2}}+\frac{Q_{1}}{s_{12}}+\frac{\left(\tau_{1}-1\right) Q_{W}^{2}}{2 s_{234}}\right)\left(\frac{\delta_{14}}{s_{14} s_{134}}-\frac{\delta_{23}}{s_{23} s_{123}}\right)\right] \\
=-\left.\mathcal{J}_{3}^{W-W^{+}}\right|_{1 \leftrightarrow 2,3 \leftrightarrow 4},
\end{gathered}
$$




$$
\begin{gathered}
\mathcal{J}_{3}^{W^{-} Z}=\mathcal{K}_{3}^{Z} Q_{W}^{2} \delta_{34}\left|U_{12}\right|^{2} L_{3}\left(s_{12}+s_{34}+s_{14}+s_{23}\right) x_{1234} \\
\times\left[\left(\frac{\tau_{1} \cot \theta_{W}}{s_{12}-M_{W}^{2}}+\frac{L_{1}}{s_{134}}\right)\left(\frac{\delta_{14}}{s_{14} s_{124}}-\frac{\delta_{23}}{s_{23} s_{234}}\right)\right. \\
\left.+\left(\frac{\tau_{2} \cot \theta_{W}}{s_{12}-M_{W}^{2}}+\frac{L_{2}}{s_{234}}\right)\left(\frac{\delta_{14}}{s_{14} s_{134}}-\frac{\delta_{23}}{s_{23} s_{123}}\right)\right] \\
\mathcal{J}_{3}^{W^{+} Z}=\mathcal{K}_{3}^{Z} Q_{W}^{2} \delta_{34}\left|U_{21}\right|^{2} L_{3}\left(s_{12}+s_{34}+s_{14}+s_{23}\right) x_{1234} \\
\times\left[\left(\frac{\tau_{1} \cot \theta_{W}}{s_{12}-M_{W}^{2}}+\frac{L_{1}}{s_{134}}\right)\left(\frac{\delta_{14}}{s_{14} s_{124}}-\frac{\delta_{23}}{s_{23} s_{234}}\right)\right. \\
\left.+\left(\frac{\tau_{2} \cot \theta_{W}}{s_{12}-M_{W}^{2}}+\frac{L_{2}}{s_{234}}\right)\left(\frac{\delta_{14}}{s_{14} s_{134}}-\frac{\delta_{23}}{s_{23} s_{123}}\right)\right] \\
=-\left.\mathcal{J}_{3}^{W-Z}\right|_{1 \leftrightarrow 2,3 \leftrightarrow 4}
\end{gathered}
$$

We note that the equations (2.81) and (2.82) are formally identical (except for the change $\left.U \rightarrow U^{\dagger}\right)$, but the two expressions are not equal because the flavors involved are different. The same remark holds for the following two equations:

$$
\begin{gathered}
\mathcal{J}_{3}^{Z W^{+}}=\mathcal{K}_{3}^{W} Q_{W}^{2} \delta_{14} \delta_{23}\left|U_{21}\right|^{2}\left(s_{12}+s_{34}+s_{14}+s_{23}\right) x_{1234} \\
\times\left[\left(\frac{\tau_{1} \cot \theta_{W}}{s_{12}-M_{W}^{2}}+\frac{L_{1}}{s_{234}}\right)\left(\frac{L_{2}}{s_{14} s_{134}}-\frac{L_{1}}{s_{23} s_{123}}\right)\right. \\
\left.+\left(\frac{\tau_{2} \cot \theta_{W}}{s_{12}-M_{W}^{2}}+\frac{L_{2}}{s_{134}}\right)\left(\frac{L_{2}}{s_{14} s_{124}}-\frac{L_{1}}{s_{23} s_{234}}\right)\right] \\
\mathcal{J}_{3}^{Z W^{-}}=\mathcal{K}_{3}^{W} Q_{W}^{2} \delta_{14} \delta_{23}\left|U_{12}\right|^{2}\left(s_{12}+s_{34}+s_{14}+s_{23}\right) x_{1234} \\
\times\left[\left(\frac{\tau_{1} \cot \theta_{W}}{s_{12}-M_{W}^{2}}+\frac{L_{1}}{s_{234}}\right)\left(\frac{L_{2}}{s_{14} s_{134}}-\frac{L_{1}}{s_{23} s_{123}}\right)\right. \\
\left.\quad+\left(\frac{\tau_{2} \cot \theta_{W}}{s_{12}-M_{W}^{2}}+\frac{L_{2}}{s_{134}}\right)\left(\frac{L_{2}}{s_{14} s_{124}}-\frac{L_{1}}{s_{23} s_{234}}\right)\right] \\
=-\left.\mathcal{J}_{3}^{Z W^{+}}\right|_{1 \leftrightarrow 2,3 \leftrightarrow 4}
\end{gathered}
$$

Finally,

$$
\begin{aligned}
& \mathcal{J}_{3}^{Z Z}=\mathcal{K}_{3}^{Z} \delta_{12} \delta_{14} \delta_{23}\left(L_{1}^{4}-R_{1}^{4}\right)\left(s_{12}+s_{34}+s_{14}+s_{23}\right) x_{1234} \\
& \quad \times\left[\frac{1}{s_{134}}\left(\frac{1}{s_{14} s_{124}}-\frac{1}{s_{23} s_{234}}\right)+\frac{1}{s_{234}}\left(\frac{1}{s_{14} s_{134}}-\frac{1}{s_{23} s_{123}}\right)\right] .
\end{aligned}
$$

\section{Three particle final states: $\left(V_{q} \rightarrow q_{3} \bar{q}_{4}\right)+\gamma$}

In this subsection we present formulas for the chromo-electroweak contributions $\mathcal{J}_{3}^{\gamma V_{q}}$ defined in Eq. (2.77) to the squares of the amplitudes for real photon production which were 
presented in the preceding subsection. The squared amplitudes can be compactly written in terms of the functions

$$
\begin{aligned}
& X_{12345}^{\gamma} \equiv \frac{H_{12345} H_{32145}^{+}}{s_{14}} \\
& Y_{12345}^{\gamma} \equiv \frac{H_{12345} H_{14325}^{+}}{s_{23}} .
\end{aligned}
$$

Only the imaginary parts of these functions contribute to $\mathcal{J}_{3}^{\gamma V_{q}}$; in Appendix B we show that

$$
\begin{aligned}
& \operatorname{Im} X_{12345}^{\gamma}=16 \frac{s_{13}^{2}}{s_{14} s_{15} s_{25} s_{35}} x_{1234}, \\
& \operatorname{Im} Y_{12345}^{\gamma}=16 \frac{s_{13}^{2}}{s_{23} s_{15} s_{25} s_{45}} x_{1234} .
\end{aligned}
$$

From Eqs. (2.35) to (2.48), we obtain

$$
\begin{aligned}
\mathcal{J}_{3}^{\gamma W^{+}}= & \mathcal{K}_{3}^{W} Q_{W}^{2}\left|U_{21}\right|^{2} \delta_{14} \delta_{23}\left(s_{13}^{2}-s_{24}^{2}\right) x_{1234} \\
& \times \frac{1}{s_{15}}\left(\frac{\tau_{2}}{s_{12}-M_{W}^{2}}+\frac{Q_{2}}{s_{25}}\right)\left(\frac{Q_{1}}{s_{23} s_{45}}+\frac{Q_{2}}{s_{14} s_{35}}\right), \\
\mathcal{J}_{3}^{\gamma W^{-}}= & \mathcal{K}_{3}^{W} Q_{W}^{2}\left|U_{12}\right|^{2} \delta_{14} \delta_{23}\left(s_{13}^{2}-s_{24}^{2}\right) x_{1234} \\
& \times \frac{1}{s_{25}}\left(\frac{\tau_{1}}{s_{12}-M_{W}^{2}}+\frac{Q_{1}}{s_{15}}\right)\left(\frac{Q_{1}}{s_{23} s_{45}}+\frac{Q_{2}}{s_{14} s_{35}}\right) \\
= & -\left.\mathcal{J}_{3}^{\gamma W^{+}}\right|_{1 \leftrightarrow 2,3 \leftrightarrow 4}, \\
\mathcal{J}_{3}^{\gamma Z}= & \mathcal{K}_{3}^{Z} Q_{1}^{2} \delta_{12} \delta_{14} \delta_{23}\left(L_{1}^{2}-R_{1}^{2}\right)\left(s_{13}^{2}-s_{24}^{2}\right) x_{1234} \\
& \times \frac{1}{s_{15} s_{25}}\left(\frac{1}{s_{23} s_{45}}+\frac{1}{s_{14} s_{35}}\right) .
\end{aligned}
$$

\section{PARITY-VIOLATING ASYMMETRIES}

\section{A. General Definition}

The chromo-electroweak interference contributions to the cross section for producing a vector boson plus two jets which were presented above are odd under space-reflection. A simple way of defining experimental observables that are sensitive to this parity-odd character of the interference terms is as follows: Imagine that the incident beams lie in the plane of a mirror. If the incident beams are not polarized, the initial state is invariant under 
reflection in this mirror. We will also assume that the spins of the final state particles are not detected, i.e., particles are identified by their momenta and internal quantum numbers only. A parity-odd contribution to the cross section can make the probabilities for observing a particular event (i.e., a particular configuration of final state particles) and its mirror image different from one another. Since the events are continuously distributed in phase space, the likelihood of finding an event and its geometrical mirror image in any finite sample of events is vanishingly small. To decide experimentally whether or not there is an asymmetry with respect to mirror reflection in the event sample, one must count the number of events that fall in some region of phase space (which we will call a "bin") and the number of events that fall in the mirror image of this region (which we will the "image bin") and then decide whether or not there is a statistically significant difference between these two numbers. This difference can be compared with a "parity-violating asymmetry" which we define as follows:

$$
a^{\mathrm{pv}}(\operatorname{bin})=\int_{\text {bin }} d \sigma-\int_{\text {image bin }} d \sigma .
$$

The integral over the bin includes an implicit sum over all final state quantum numbers that are not observed, i.e., color quantum numbers and any spin or flavor quantum numbers that cannot be experimentally measured. Note that the parity-even terms in the differential cross section $d \sigma$ do not contribute to $a^{\mathrm{pv}}$, which therefore provides a direct measure of the parity-odd terms.

The asymmetry $a^{\mathrm{pv}}($ bin) will obviously depend on the location of the chosen bin in phase space and on its size and shape. It will be small if the bin is very small. It will obviously be zero if the bin consists of the whole phase space, or if the bin is invariant under mirror reflection. Note also that events which lie in any portion of a bin that overlaps with a portion of the image bin will not contribute to $a^{\mathrm{pv}}$. While such "symmetric" events do not contribute to the size of the asymmetry, they will tend to exaggerate its statistical significance. We will therefore restrict the chosen bins to those which do not overlap with their mirror images.

A set of bins will have to be judiciously chosen to maximize the observed effects of parity violation, i.e., to yield the largest cumulative asymmetry. Thus we also define a cumulative parity-violating asymmetry as follows:

$$
A^{\mathrm{pv}}=\sum_{\text {bins }}\left|a^{\mathrm{pv}}(\mathrm{bin})\right|
$$

where the sum is taken over bins that do not overlap with one another. To avoid counting events more than once we will also demand that the bins be chosen so that no bin in this sum overlaps with the mirror image of any other bin. $A^{\mathrm{pv}}$ will obviously be largest if $a^{\mathrm{pv}}$ can be measured in a large number of small bins. The size of the total event sample will put a lower limit on the number of bins and their sizes since the asymmetry in each bin-image pair must be statistically significant.

A theoretical upper bound on $A^{\mathrm{pv}}$ is given by

$$
A_{\mathrm{max}}^{\mathrm{pv}}=\sum_{\text {bins }} \lim _{\text {bin size } \rightarrow 0}\left|a^{\mathrm{pv}}(\mathrm{bin})\right| \text {. }
$$

If $d \sigma / d \Omega$ is the fully differential cross section (which includes a sum over unobserved quantum numbers), 


$$
A_{\max }^{\mathrm{pv}}=\frac{1}{2} \int_{<\mathrm{cuts}>} d \Omega\left|\left(\frac{d \sigma}{d \Omega}\right)-\left(\frac{d \sigma}{d \Omega}\right)_{\text {mirror image }}\right|
$$

The factor of $\frac{1}{2}$ ensures that no event is counted twice. The region of integration is taken to be all of the phase space that satisfies cuts that are mandated by experimental considerations, and by the need to exclude regions where the theoretical expressions for $d \sigma / d \Omega$ become unreliable due to non-perturbative effects. The cuts will be taken to be invariant under mirror-reflection: this assumption will not unduly restrict the event sample in a collidingbeam experiment with a $4 \pi$ or a cylindrically symmetrical detector.

\section{B. Asymmetries in Electroweak Boson +2 Jet Production}

In this subsection we will apply the general definition of parity-violating asymmetries introduced above to the production of an electroweak boson and two jets. We first describe the kinematics appropriate to the three-particle final state at the parton level. We will then define and present results for asymmetries at the parton and the hadron levels.

\section{Kinematics}

We assume that the colliding beams are collinear and define a $z$ axis. The colliding partons are assumed to have momenta $p_{1}$ and $p_{2}$, with $\vec{p}_{1}$ in the positive $z$-direction. We assume that the electroweak boson has momentum $q$, and choose the $x$-axis so that $\vec{q}$ lies in the $x-z$ plane and $q^{x}>0$. (We assume that an on-shell $W$ or $Z$ is detected in its leptonic decay mode, and that its momentum has been reconstructed from measurement of the decay-lepton momenta.) The $y$ axis is chosen so that the coordinate system is righthanded as illustrated in Fig. 6. The momenta of the two final state partons (jets) will be denoted by $p_{3}$ and $p_{4}$.

If $\mathcal{J}_{3}$ is a contribution to a spin and helicity averaged squared matrix element, the corresponding contribution to the differential cross section at the parton level is

$$
d \hat{\sigma}=\frac{1}{2 s_{12}}(2 \pi)^{4} \delta^{4}\left(p_{1}+p_{2}-q-p_{3}-p_{4}\right) \mathcal{J}_{3} \mathcal{B}_{\text {lep }} \frac{d^{3} p_{3} d^{3} p_{4} d^{3} q}{(2 \pi)^{9} 8 p_{3}^{0} p_{4}^{0} q^{0}} .
$$

In this equation we have explicitly included a factor of the leptonic branching ratio $\mathcal{B}_{\text {lep }}$ of the electroweak boson (i.e., $\mathcal{B}_{\text {lep }}=\Gamma(W \rightarrow e \nu) / \Gamma_{\text {tot }}$ for $W^{ \pm}, \Gamma\left(Z \rightarrow e^{+} e^{-}\right) / \Gamma_{\text {tot }}$ for $Z^{0}$, and 1 for a real photon). We note that the subscripts labeling the parton momenta $p_{i}$ stand for particular points in momentum space and not for parton quantum numbers. To be more precise, let us write the parton level reaction as follows:

$$
a_{1}\left(p_{1}\right)+a_{2}\left(p_{2}\right) \rightarrow a_{3}\left(p_{3}\right)+a_{4}\left(p_{4}\right)+V_{l}(q),
$$

where $a_{i}$ stand for parton flavors. The quantities $\mathcal{J}_{3}$ defined in Eq. (2.77) were functions of momenta $k_{i}, i=1, \ldots 4$ all of which were taken to be outgoing, and they also depended on the flavors $f_{i}$ of the quarks or antiquarks which carried these momenta. We express this dependence explicitly as follows: 


$$
\mathcal{J}_{3}^{V_{l} V_{q}}\left(f_{1}\left(k_{1}\right), f_{2}\left(k_{2}\right), f_{3}\left(k_{3}\right), f_{4}\left(k_{4}\right)\right)
$$

Partons $f_{1}$ and $f_{2}$ must be crossed to the initial state, and then either one of them can be identified with the beam parton $a_{1}\left(p_{1}\right)$. Parton $f_{3}$ can then be assigned either a momentum $p_{3}$ or a momentum $p_{4}$. For example if $V_{l}=Z^{0}, V_{q}=W^{+}$, we have the following possibilities:

$$
\begin{aligned}
& u\left(p_{1}\right)+\bar{d}\left(p_{2}\right) \rightarrow u\left(p_{3}\right)+\bar{d}\left(p_{4}\right)+Z^{0} \Rightarrow \mathcal{J}_{3}^{Z W^{+}}\left(d\left(-p_{2}\right), \bar{u}\left(-p_{1}\right), u\left(p_{3}\right), \bar{d}\left(p_{4}\right)\right), \\
& \bar{d}\left(p_{1}\right)+u\left(p_{2}\right) \rightarrow u\left(p_{3}\right)+\bar{d}\left(p_{4}\right)+Z^{0} \Rightarrow \mathcal{J}_{3}^{Z W^{+}}\left(d\left(-p_{1}\right), \bar{u}\left(-p_{2}\right), u\left(p_{3}\right), \bar{d}\left(p_{4}\right)\right), \\
& u\left(p_{1}\right)+\bar{d}\left(p_{2}\right) \rightarrow \bar{d}\left(p_{3}\right)+u\left(p_{4}\right)+Z^{0} \Rightarrow \mathcal{J}_{3}^{Z W^{+}}\left(d\left(-p_{2}\right), \bar{u}\left(-p_{1}\right), u\left(p_{4}\right), \bar{d}\left(p_{3}\right)\right), \\
& \bar{d}\left(p_{1}\right)+u\left(p_{2}\right) \rightarrow \bar{d}\left(p_{3}\right)+u\left(p_{4}\right)+Z^{0} \Rightarrow \mathcal{J}_{3}^{Z W^{+}}\left(d\left(-p_{1}\right), \bar{u}\left(-p_{2}\right), u\left(p_{4}\right), \bar{d}\left(p_{3}\right)\right) .
\end{aligned}
$$

In these expressions, $u$ and $d$ stand for generic weak $I_{z}= \pm \frac{1}{2}$ flavors, i.e., " $u$ " $=u, c, \ldots$ and " $d "=d, s, b, \ldots$, etc. In this paper we will include the contributions of the "lighter" flavors $u, d, s, c, b$ only in making numerical predictions.

Since we have used the narrow width approximation for $V_{q}$, see Eq. (2.58), let us write

$$
\mathcal{J}_{3}=\hat{\mathcal{J}}_{3} \delta\left(s_{34}-M_{V_{q}}^{2}\right) .
$$

Because the initial partons are assumed unpolarized, $\mathcal{J}_{3}$ is invariant under rotations about the $z$-axis. The 3-particle phase space in Eq. (3.5) is thus effectively 3-dimensional. We will choose the rapidity $y_{3}$ and azimuthal angle $\phi_{3}$ of the parton with momentum $p_{3}$ and the rapidity $y_{q}$ of $V_{l}$ as the three independent phase space variables. The rapidities are invariant under reflection in the $x-z$ plane, while $\phi_{3} \rightarrow 2 \pi-\phi_{3}$. Performing the trivial integrations we obtain

$$
\frac{d \hat{\sigma}}{d y_{q} d y_{3} d \phi_{3}}=\frac{p_{3 T}^{2} E_{q T}^{2} \hat{\mathcal{J}}_{3} \mathcal{B}_{\text {lep }}}{2^{7} \pi^{4} s_{12} s_{34}\left(s_{12}+M_{V_{l}}^{2}-s_{34}\right)},
$$

where

$$
\begin{aligned}
& E_{q T} \equiv \sqrt{q_{x}^{2}+q_{y}^{2}+M_{V_{l}}^{2}}=\frac{s_{12}+M_{V_{l}}^{2}-M_{V_{q}}^{2}}{2 \sqrt{s_{12}} \cosh \left(y_{12}-y_{q}\right)}, \\
& p_{3 T} \equiv \sqrt{p_{3 x}^{2}+p_{3 y}^{2}}=\frac{M_{V_{q}}^{2}}{2\left[\sqrt{s_{12}} \cosh \left(y_{12}-y_{3}\right)-E_{q T} \cosh \left(y_{q}-y_{3}\right)+q_{T} \cos \phi_{3}\right]}, \\
& q_{T} \equiv \sqrt{q_{x}^{2}+q_{y}^{2}}, \quad y_{12} \equiv \frac{1}{2} \ln \left(\frac{p_{1}^{0}+p_{2}^{0}+p_{1}^{z}+p_{2}^{z}}{p_{1}^{0}+p_{2}^{0}-p_{1}^{z}-p_{2}^{z}}\right) .
\end{aligned}
$$

The parton subprocess (3.6) will contribute to the cross section for the hadronic process $h_{1}\left(P_{1}\right)+h_{2}\left(P_{2}\right) \rightarrow V_{l}+2$ Jets:

$$
\frac{d \sigma}{d \tau d y_{12} d y_{q} d y_{3} d \phi_{3}}=\sum_{a_{1} a_{2}} f_{a_{1} / h_{1}}\left(x_{1}, Q^{2}\right) f_{a_{2} / h_{2}}\left(x_{2}, Q^{2}\right) \frac{d \hat{\sigma}}{d y_{q} d y_{3} d \phi_{3}},
$$

where

$$
\tau=x_{1} x_{2}=\frac{s_{12}}{s}, \quad s=\left(P_{1}+P_{2}\right)^{2}, \quad y_{12}=\frac{1}{2} \ln \left(\frac{x_{1}}{x_{2}}\right),
$$


and $f_{a / h}\left(x, Q^{2}\right)$ is the density of partons of flavor $a$ and momentum fraction $x$ in the hadron $h$. In this paper we will use the structure function parametrizations of Martin, Roberts and Stirling [17] (set MRSB). The momentum scale $Q$ of the parton densities is not precisely determined since we have not computed the next-to-leading corrections to the cross section, so we will take $Q^{2}=s_{12} / 4$ in obtaining numerical predictions.

We next specify a set of cuts that we will use when we integrate the differential cross section to obtain the asymmetries defined in Eqs. (3.1) through (3.4). We will apply a single representative set of cuts on the momenta of the final state particles for most of the numerical results presented in this paper. These cuts are defined in terms of the transverse momenta $p_{i T}$, pseudorapidities $\eta_{i}=-\ln \tan \left(\theta_{i} / 2\right)$ and azimuthal angles $\phi_{i}$, in the laboratory frame. The index $i$ runs over $V_{l}, j_{1}$ and $j_{2}$ for three-particle final states, and over $l, \bar{l}, j_{1}$ and $j_{2}$ for four-particle final states. We choose

$$
\begin{aligned}
\text { (i) } & p_{i T} \geq 10 \mathrm{GeV} \\
\text { (ii) } & \left|\eta_{i}\right| \leq 2.5 \\
\text { (iii) } & {\left[\left(\eta_{i}-\eta_{j}\right)^{2}+\left(\phi_{i}-\phi_{j}\right)^{2}\right] \geq 0.7 . }
\end{aligned}
$$

These cuts are meant to ensure that the jets are well resolved experimentally, and to exclude regions of phase space where the theoretical predictions are likely to become unreliable due to infrared and collinear singularities. We will use the Monte Carlo program veGAs [18 to perform the phase space integrations.

\section{Parton-level Asymmetries}

To illustrate the nature and magnitude of the parity-violating effects, we first present results for the differential asymmetry $d a^{\mathrm{pv}} / d \phi_{3}$ that would be observed in a quark-antiquark collisions if different quark flavors could be distinguished from one another. We assign the incoming quark the momentum $p_{1}$ and the outgoing quark the momentum $p_{3}$. According to Eq. (3.1), the differential asymmetry represents the excess of events/degree in which the final state quark is observed at an azimuthal angle $\phi_{3}$ over those in which it is observed at an angle $2 \pi-\phi_{3}$. The asymmetry at an angle $\phi_{3} \geq 180^{\circ}$ is the negative of that at the complementary angle $2 \pi-\phi_{3}$.

Fig. [7a shows differential asymmetries for the case $V_{l}=W^{-}$. There are four possible

subprocesses, two with $V_{q}=Z^{0}$ and two with $V_{q}=W^{+}$. The asymmetry is largest when the quark is emitted almost diametrically opposite in azimuth to the $W$. It is zero when $\phi_{3}=0$ or $180^{\circ}$ because the 5 particles involved in the collision are then coplanar, and the parityviolating invariant $x_{1234}$ vanishes. In Fig. $7 \mathrm{~b}$ we present asymmetries for the two processes with $V_{q}=Z^{0}$, with various assumptions. The solid curve, reproduced from Fig. 7a, is for the subprocess $d \bar{u} \rightarrow u \bar{u} W^{-}$with the $u$ quark observed at angle $\phi_{3}$. The dotted curve is for observation of the $\bar{u}$ at angle $\phi_{3}$. If one is unable to distinguish quarks from antiquarks, one must add the corresponding cross sections: this is shown by the dashed curve. The subprocess $d \bar{u} \rightarrow d \bar{d} W^{-}$can also contribute if one cannot distinguish $u$ from $d$ quark jets: the dot-dashed curve shows the sum of the asymmetries in which the jet observed at angle $\phi_{3}$ can come from $u, \bar{u}, d$ or $\bar{d}$. The figure shows that the inability to distinguish flavors 
experimentally in a jet may reduce the asymmetry, but does not necessarily reduce it to zero.

In Fig. 8 we present differential asymmetries for the cases $V_{l}=W^{+}, Z^{0}$, and in Fig. 9 for $V_{l}=\gamma$. From Eqs. 2.79)-(2.82) we see that the processes contributing to Figs. $7 \mathrm{a}$ and $8 \mathrm{a}$ are related by $\mathrm{CP}$ conjugation. We therefore expect the asymmetries in Fig. $7 \mathrm{a}$ to be the same as asymmetries for $W^{+}$production if the produced antiquark is observed at angle $\phi_{3}$, the relative minus sign between the cross sections being compensated by the fact that we assign the incoming quark the momentum $p_{1}$ in both cases. However, Fig. Ba shows asymmetries with the quark observed at angle $\phi_{3}$ : these are opposite in sign and in general different in magnitude from the corresponding charge conjugate processes in Fig. $7 \mathrm{a}$. It is interesting that the asymmetries for the processes $u \bar{u} \rightarrow u \bar{d} W^{-}$and $d \bar{d} \rightarrow d \bar{u} W^{+}$are numerically almost equal, as are the asymmetries for the CP conjugate pair of processes. The asymmetries in Fig. 8b for $Z^{0}$ production are interesting in that they exhibit zeros at intermediate values of $\phi_{3}$. The figures also show that the asymmetries when one or both of the vector bosons are $Z$ s tend to be smaller than when they are $W$ s. Finally, Fig. 9 shows that the asymmetries in the case of real photon production are almost an order of magnitude larger than for production of massive vector boson pairs. This difference is partly due to the fact that the threshold for real photon production is lower than that for vector boson pair production. Fig. 9a is plotted for a subprocess energy of $110 \mathrm{GeV}$ around which the asymmetries tend to be largest. Fig. 9b shows that the asymmetries at $250 \mathrm{GeV}$, which is a little above the weak boson pair production threshold, are similar in magnitude to those in Figs. 7 and 8.

We next present results in Figs. $10-12$ for the maximum observable cumulative asymmetries $A_{\max }^{\mathrm{pv}}$ that were defined in Eqs. (3.3) and (3.4). (The results for $p p$ and $p \bar{p}$ collisions, which are presented for convenience in the same figures, will be discussed in the following sub-section.) We remind the reader that the maximum asymmetry is an upper bound on the cumulative observable asymmetry, a bound which can be saturated only if one surrounds the interaction region with detectors that are sufficiently small that the fully differential asymmetry (in all kinematic variables and not just $\phi_{3}$ ) does not change sign within the acceptance of each of them. These figures show that the asymmetries are largest at a few tens of $\mathrm{GeV}$ above the threshold for production of the vector boson pair, and that they then fall quite rapidly with energy. We first discuss the two parton-level curves in Fig. 7a. The integrated value of the differential asymmetry for the process $u \bar{u} \rightarrow u \bar{d} W^{-}$shown by the dashed curve in Fig. $7 \mathrm{a}$ is $-0.0201 \mathrm{pb}$, and the corresponding value of $A_{\max }^{\mathrm{pv}}$ from the dashed curve in Fig. 10a is $0.0225 \mathrm{pb}$. This comparison shows that oscillations in sign of the asymmetry in variables other than the angle $\phi_{3}$ are negligible. The dot-dashed curve in Fig. 10a shows the "flavor-blind" asymmetry for this subprocess. We emphasize the fact that in forming this asymmetry, the fully-differential cross sections for $u$ and for $\bar{d}$ to be observed at azimuth $\phi_{3}$ are added before taking the absolute magnitude in Eq. (3.4). The corresponding parton-level cross sections for the process $d \bar{d} \rightarrow u \bar{d} W^{-}$are numerically very close to the cross sections for $u \bar{u} \rightarrow u \bar{d} W^{-}$, and are therefore not shown. Fig. 10p shows $A_{\mathrm{max}}^{\mathrm{pv}}$ for the processes $d \bar{u} \rightarrow Z^{0} W^{-}$, with $V_{q}=Z^{0} \rightarrow u \bar{u}$ or $d \bar{d}$. The five parton-level curves give maximum asymmetries with various assumptions concerning distinguishability of quark jets. It is apparent that there is very little cancellation between asymmetries for processes with quarks and antiquarks or quarks with different weak-isospin quantum numbers; thus if 
any two of these processes produce asymmetries of opposite sign, these must occur in different regions of phase space. Maximum asymmetries in the production of a $Z^{0}$ are presented in Fig. 11, and for photon production in Fig. 12. A comparison of Figs. $10-12$ shows that the largest asymmetries occur in the case of a real photon and in particular when $V_{q}=W^{ \pm}$.

\section{Hadron-level asymmetries}

The parton-level asymmetries presented in the preceding section will produce asymmetries at the hadron-level in the processes $p \bar{p}$ or $p p \rightarrow V_{l}+2$ Jets according to Eq. (3.11). In obtaining numerical predictions for these asymmetries we shall assume that the invariant mass of the 2-jet system can be reconstructed to within approximately $5 \mathrm{GeV}$ so that jets arising from the resonant decay of a $W$ can be distinguished from jets arising from the decay of a $Z$. We will take into consideration the contributions of five quark flavors $u, d, s, c$, and $b$, using the MRSB structure function parametrizations [17] to compute parton densities $f_{a / h}\left(x, Q^{2}\right)$. We will assume that the flavors of parton jets cannot be measured, and will sum differential asymmetries over all possible incoming and outgoing light quark (and antiquark) flavors.

Figs. 10 12 show maximum observable cumulative asymmetries $A_{\max }^{\mathrm{pv}}$ for $V_{l}=W, Z$ or $\gamma$. The asymmetries begin to be appreciable at values of $\sqrt{s}$ approximately 6 times larger than the threshold for pair production of the corresponding pair of vector bosons. They are initially larger for $p \bar{p}$ collisions than for $p p$ collisions because the contributing subprocesses involve quark-antiquark annihilation. As $\sqrt{s}$ increases, the densities of sea quarks increase relative to those of valence quarks, and the $p p$ and $p \bar{p}$ curves get closer to one another. A comparison of Figs. 10 and 11 with Fig. 12 would suggest that the prospects for observing parity-violating asymmetries might be best in the case of real photon production; however, the QCD background is also larger for real photon production than it is for $W$ or $Z$ production, as we shall see in the next sub-section. It should be emphasized that the cross sections in Figs. 10 and 11 for production of a "real" $W$ or $Z$ have been multiplied by the appropriate leptonic branching ratio (see Eq. (3.5)) for a single leptonic decay mode. If more than one leptonic decay mode is observed, the signal will be correspondingly enhanced.

In Fig. 13a we present binned asymmetries as defined in Eq. (3.1) for $W^{-}$and $Z$ production in $p \bar{p}$ collisions at $2 \mathrm{TeV}$. We have chosen bins of width $10^{\circ}$ in the angle $\phi_{3}$ and $500 \mathrm{GeV}$ in the subprocess invariant mass $\sqrt{s_{12}}$. Fig. 13b shows corresponding results for real photon production. We note with reference to Fig. 6 that the proton beam has been chosen to define the positive $z$ direction: thus, with a right handed coordinate system, a positive asymmetry implies an excess of events in which the jet assigned a momentum $p_{3}$ and azimuthal angle $\phi_{3}$ has a positive $y$ component of momentum. Obviously, the existence of a non-zero asymmetry depends on the fact that the proton and antiproton beams differ from one another; specifically, they differ in their valence quark content, and this produces a physical distinction between the positive and negative $z$ directions, and thus the handedness of the coordinate system is related to physical observables. If one sums the asymmetries represented by any of the histograms in Fig. 13 algebraically, one obtains a zero net asymmetry. This is due to the fact that we have assumed that the jets are identified only by their momenta: momentum conservation requires that if one of the jets has a positive $y$ component of momentum, then the other has a negative $y$ component; if the bin encom- 
passes the whole of the hemisphere with $p_{3}^{y} \geq 0$, no observed quantum number distinguishes between the two hemispheres, and the asymmetry must vanish. We note finally that the cumulative asymmetries as defined by Eq. (3.2) for any of the histograms in these figures are typically of order $20 \%$ of the corresponding maximum observable asymmetries in Figs. $10-12$ for $p \bar{p}$ collisions at $2 \mathrm{TeV}$. For example, the cumulative asymmetry $A^{\mathrm{pv}}$ from the solid-line histogram in Fig. $13 \mathrm{a}$ is $0.010 \mathrm{pb}$, while $A_{\max }^{\mathrm{pv}}$ from the solid-line curve in Fig. 10 at $2 \mathrm{TeV}$ is $0.033 \mathrm{pb}$. There are evidently considerably larger cancellations at the hadron level than at the parton level in the bins we have chosen. These cancellations presumably occur in the sums over parton types and the integrations over parton momentum fractions, see Eq. (3.11), required to compute the hadronic cross sections. It is rather remarkable nevertheless that such a substantial signal of the parton-level asymmetry survives at the hadron level.

We next discuss asymmetries in $p p$ collisions. It is apparent in this case that the colliding beams will not provide a means for physically distinguishing the positive and negative $z$ directions, since their valence and sea quark contents are identical. Thus if there were no other observable that distinguishes the $\pm z$ directions, as was for instance the case for the binned asymmetries in $p p$ collisions presented in Fig. 13, one would observe no parity violation. To observe a non-zero asymmetry, there must exist an observable that is related to the handedness of the coordinate system. The simplest way to introduce such an observable is to demand that the vector boson have a positive $z$ component of momentum in the laboratory (center-of-mass frame of the colliding beams) in which the positive $z$ axis has been chosen to coincide with one of the two proton beams chosen arbitrarily. In other words, we choose bins for which the rapidity $y_{q}$ is $\geq 0$. We could also choose bins in which $y_{q} \leq 0$, or in general, bins that are not symmetric in rapidity. Fig. 14 shows the binned asymmetries

that one obtains for $p p$ collisions at $40 \mathrm{TeV}$ in the case of real photon production with $y_{q} \geq 0$ as well as for $y_{q} \leq 0$. It is evident that the sum of the two histograms vanishes (within the uncertainties of the Monte Carlo integration). It is evidently also possible to choose bins that are asymmetric in the rapidity $y_{3}$ of the jet assigned the momentum $p_{3}$, and these asymmetries are also shown in the figure. In Fig. 15 results are presented for $W^{-}$and $Z^{0}$ production in $p p$ collisions at $40 \mathrm{TeV}$.

\section{Parity-violating signal and $Q C D$ background}

The parity-violating asymmetries presented above will in practice be concealed in a substantial parity-conserving background of electroweak boson +2 jet events [14]. This QCD background completely overwhelms the signal from electroweak boson pair production in which one of the two bosons is observed in its leptonic decay modes, and the other decays to two hadronic jets: Stirling, Kleiss and Ellis [19] found that the signal to background ratio varies from $\sim 1 / 30$ at $2 \mathrm{TeV}$ to $\sim 1 / 150$ at $40 \mathrm{TeV}$ in $p \bar{p}$ collisions. We find that similar signal to background ratios occur for the chromo-electroweak interference contributions. It should nevertheless be possible in principle to observe the interference contributions by measuring parity-violating asymmetries to which the QCD background does not contribute. In practice, there will be statistical fluctuations in the background which will tend to obscure such a signal: it is therefore essential to estimate the signal to background ratio in order to decide whether such asymmetries will be measurable. 
To estimate the QCD background we use a set of compact analytic cross sections computed by R.K. Ellis and one of us (R.J.G.) [20]. We apply the same set of cuts given in Eqs. (3.14) to the QCD background events that we have used to compute the asymmetries. In computing the latter, we have used the narrow width approximation defined in Eq. (2.58) for the propagator of the the electroweak boson $V_{q}$ which decays to two jets. There is of course no such propagator in the amplitudes for the QCD background, and the observed events are therefore expected to vary quite slowly as a function of the invariant mass $\sqrt{s_{34}}$ of the two jets with a small blip coming from the $V_{q}$ propagator in the pair-production and interference contributions at $\sqrt{s_{34}}=M_{V_{q}}^{2}$. To extract this blip, events need only be sampled in a small interval $\left(M_{V_{q}}-\Delta\right)^{2} \leq s_{34} \leq\left(M_{V_{q}}+\Delta\right)^{2}$, with $\Delta \simeq \Gamma_{V_{q}}$. In computing the background, we therefore apply an additional cut $\left(M_{V_{q}}-\Gamma_{V_{q}}\right)^{2} \leq s_{34} \leq\left(M_{V_{q}}+\Gamma_{V_{q}}\right)^{2}$ on the jet-jet invariant mass $\sqrt{s_{34}}$.

Figures $16-18$ show comparisons of the parity-violating signal with the QCD background in electroweak boson +2 jet production. The signal to background ratios are rather small. They are generally largest at $\sqrt{s} \sim 1 \mathrm{TeV}$, and decrease with increasing $\sqrt{s}$ because the QCD background grows more rapidly than the asymmetries. The magnitude and trend of the signal to background ratios are similar to those observed in electroweak boson pair production [19,15]. Some possible reasons why a larger resonant-peak effect is not observed were discussed in the paragraphs following Eq. (2.3). On a positive note, it is fairly remarkable that a signal comparable in magnitude to the pair-production cross section does survive, in spite of the fact that we have imposed absolutely no requirements concerning detection of the spins or flavors of the parton jets.

Let us next estimate the event rates required to observe these asymmetries in $p \bar{p}$ collisions at $\sqrt{s}=2 \mathrm{TeV}$, for example. Suppose that one has chosen a bin as defined in Fig. 6. Let $n_{\text {bin }}$ and $n_{\text {image bin }}$ be the number of experimentally observed events in this bin and its image, respectively. Then,

$$
a^{\mathrm{pv}}(\text { bin })=\frac{n_{\text {bin }}-n_{\text {image bin }}}{\int \mathcal{L}}
$$

where $\int \mathcal{L}$ is the integrated luminosity used to obtain the sample of events. The difference $n_{\text {bin }}-n_{\text {image bin }}$ will be significant only if it is larger in absolute magnitude than statistical fluctuations of order $n_{\text {bin }}^{1 / 2}$ in the number of events measured in the bin. This means that an integrated luminosity

$$
\int \mathcal{L} \gtrsim \frac{\sigma_{\mathrm{QCD}}}{\left[a^{\mathrm{pv}}\right]^{2}}
$$

is required to observe the parity-violating asymmetry in any particular bin.

To estimate typical integrated luminosities required, consider Fig. 16. Choose a bin such that $140^{\circ}<\phi_{3}<180^{\circ}$. In this bin, $a^{\mathrm{pv}}=0.0049 \mathrm{pb}$ from the solid curve with $V_{q}=W^{+}$, and $\sigma_{\mathrm{QCD}}=0.42 \mathrm{pb}$ from the dashed curve. From Eq. (3.15), an integrated luminosity of $17(\mathrm{nb})^{-1}$ would be required to observe this asymmetry. Approximately $13(\mathrm{nb})^{-1}$ would be required for a bin spanning $0^{\circ}<\phi_{3}<140^{\circ}$ where the signal to background ratio is smaller. If it is assumed that $a^{\mathrm{pv}}$ changes sign around $\phi_{3}=140^{\circ}$, approximately $7.6(\mathrm{nb})^{-1}$ would be required to obtain a statistically significant signal of parity violation from the whole histogram. It appears quite feasible to collect samples on the order of $5-25(\mathrm{pb})^{-1}$ in studies 
of $W+2$ jet events at the Fermilab Tevatron [21], and improvements in the beam luminosity might make it feasible to collect samples of order $100(\mathrm{pb})^{-1}$. However, it is apparent that very much higher integrated luminosites on the order of several (nb) ${ }^{-1}$ would be required to detect the parity-violating asymmetries studied in this paper.

Similarly large event rates are required to observe asymmetries in $Z^{0}$ and real photon production. While the predicted asymmetries in $\gamma$ production (see e.g., Fig. 18a) are approximately an order of magnitude larger in $p \bar{p} \rightarrow \gamma j j$ than they are in $W$ or $Z$ production, the somewhat larger signal to background ratios (see e.g., Fig. 18b), which enter quadratically in Eq. (3.15), effectively cancel this this apparent advantage.

\section{Asymmetries in Lepton-pair + 2 Jet Production}

In the preceding section we presented asymmetries for final states with three objects, an electroweak boson and two jets, produced at large transverse momentum. In this section we will present asymmetries for final states with four objects, a lepton-pair and two jets. These asymmetries are interesting because leptons and jets are detected directly experimentally, and the 4-particle final state allows for a richer variety of parity-violating signatures. However, since we will demand that the lepton pair has an invariant mass equal to that of a $W$

or $Z$ in order to obtain the largest asymmetries, the dynamical origin of the asymmetries is really the same as in the case of 3 particles.

\section{Kinematics}

Our choice of kinematic variables is a straightforward generalization of the 3-particle case. The colliding partons are assigned momenta $p_{1}$ and $p_{2}$, and the jet partons $p_{3}$ and $p_{4}$. With very little loss of generality we may assume that leptons can be distinguished from antileptons and that their momenta are measured experimentally either by directly observing a charged lepton or by inferring the momentum of a neutrino from missing momentum. (The case $Z^{0} \rightarrow \nu \bar{\nu}$ presents possible ambiguities which we will ignore). Lepton and antilepton are assigned momenta $p_{5}$ and $p_{6}$ respectively. We then define a right-handed coordinate system by choosing an $x$ axis such that $y_{5}^{x}>0$ and $y_{5}^{y}=0$.

If $\mathcal{J}_{4}$ is a contribution to a spin- and helicity-averaged squared matrix element, the corresponding contribution to the differential cross section at the parton level is

$$
d \hat{\sigma}=\frac{1}{2 s_{12}}(2 \pi)^{4} \delta^{4}\left(p_{1}+p_{2}-p_{3}-p_{4}-p_{5}-p_{6}\right) \mathcal{J}_{4} \frac{d^{3} p_{3} d^{3} p_{4} d^{3} p_{5} d^{3} p_{6}}{(2 \pi)^{12} 16 p_{3}^{0} p_{4}^{0} p_{5}^{0} p_{6}^{0}}
$$

In analogy with Eq. (3.9) we extract the delta functions that arise from our use of the narrow width approximation for $V_{l}$ and $V_{q}$ from the cross section:

$$
\mathcal{J}_{4}=\hat{\mathcal{J}}_{4} \delta\left(s_{34}-M_{V_{q}}^{2}\right) \delta\left(s_{56}-M_{V_{l}}^{2}\right)
$$

On account of these delta functions and the invariance of the cross section under rotations about the beam axis, the 4-particle phase space is effectively 5-dimensional. We will chose as independent variables the rapidities $y_{3}, y_{5}$ and $y_{6}$ and the azimuthal angles $\phi_{3}$ and $\phi_{6}$. 
We first perform the $\vec{p}_{4}$ integration using 3-momentum conservation, and then perform the $p_{3 T}, p_{5 T}$ and $p_{6 T}$ integrations using energy conservation and the delta functions from Eq. (3.17). This procedure yields the following equations for $p_{5 T}$ and $p_{6 T}$ :

$$
\begin{gathered}
p_{5 T} \cosh \left(y_{12}-y_{5}\right)+p_{6 T} \cosh \left(y_{12}-y_{6}\right)=\frac{s_{12}+s_{56}-s_{34}}{2 \sqrt{s_{12}}}, \\
p_{5 T} p_{6 T}=\frac{s_{56}}{2\left[\cosh \left(y_{5}-y_{6}\right)-\cos \left(\phi_{6}\right)\right]},
\end{gathered}
$$

where $y_{12}$ was defined in connection with Eq (3.10). It is evident that these two equations will, in general, have two sets of solutions for $p_{5 T}$ and $p_{6 T}$, both of which must be taken into account in performing the non-trivial phase space integrations. Finally, $p_{3 T}$ is determined by the equation

$$
p_{3 T}=\frac{s_{34}}{2\left[\sqrt{s_{12}} \cosh \left(y_{12}-y_{3}\right)-\sum_{i=5}^{6} p_{i T}\left\{\cosh \left(y_{i}-y_{3}\right)-\cos \left(\phi_{i}-\phi_{3}\right)\right\}\right]} .
$$

The final formula for the parton level cross section is

$$
\begin{aligned}
& \frac{d \hat{\sigma}}{d y_{3} d y_{5} d y_{6} d \phi_{3} d \phi_{6}}= \\
& \sum_{1}^{2} \frac{\left(p_{3 T} p_{5 T} p_{6 T}\right)^{2} \hat{\mathcal{J}}_{4}}{2^{12} \pi^{7} s_{12} s_{34} s_{56} \sqrt{s_{12}}\left|p_{5 T} \cosh \left(y_{12}-y_{5}\right)-p_{6 T} \cosh \left(y_{12}-y_{6}\right)\right|},
\end{aligned}
$$

where the sum is over the two solutions of Eqs. (3.18) and (3.19). The phase space boundaries are not easy to express in analytic form, but they are straightforward to implement in a Monte Carlo integration routine: given a set of values of the independent variables, one solves for the transverse momenta in Eqs. (3.18)- (3.20) and accepts solutions that are real and positive; the energies and momenta of the four final state particles are then reconstructed using momentum conservation, and unphysical solutions are rejected. Hadron cross sections are computed using a generalization of Eq. (3.11). A set of reflection-symmetric phase space cuts, see Eq. (3.14), are imposed as in the case of $V+2$ jet production.

\section{Parton- and hadron-level asymmetries}

In Figs. 19 and 20, we present parity-violating asymmetries that involve 4-particle final states. In general, the asymmetries tend to be a little smaller than those presented in the preceding section for $V_{l}+2$ jet production. This might be expected because the cuts in Eq. (3.14) applied to each of the two decay leptons are more restrictive than the same cuts applied to the single parent boson. In addition, the larger 4-particle phase space might be expected to allow for more cancellations among the interference contributions.

In Fig. 19a, we present parton-level asymmetries for the subprocess $u \bar{u} \rightarrow e^{-} \bar{\nu} u \bar{d}$, i.e., with $V_{l}=W^{-}$and $V_{q}=W^{+}$. The $e^{-}$momentum is used to define the $x-z$ plane. In the dotted-line histogram, the anti-neutrino is detected in azimuthal bins of size $10^{\circ}$, and no restrictions, apart from the standard cuts (3.14) are placed on the $u$ and $\bar{d}$ in the final state. 
In the other 4 histograms, the $u$ quark is detected in the azimuthal bin, and no restrictions, other than the standard cuts, are placed on the $\bar{\nu}$ : in the solid-line histogram, no restriction, other than the standard cuts, is placed on the $\bar{d}$; and in the remaining 3 histograms, the azimuthal angle $\phi_{4}$ of the $\bar{d}$ is restricted as indicated. The various histograms have characteristically different shapes. It is evident that a much richer variety of asymmetries can be defined in the case of 4-particle final states, when compared with the corresponding 3particle process - see the dashed curve in Fig. $7 \mathrm{a}$. It should be possible to exploit this variety to reduce statistical uncertainties in the signal-to-background ratio by rebinning an experimental sample of events in different ways. In Fig. 19b, we present hadron-level asymmetries for the process $p \bar{p} \rightarrow e^{-} \bar{\nu}+2$ jets-i.e., with $V_{l}=W^{-}$decaying to the lepton pair-at $\sqrt{s}=2 \mathrm{TeV}$. For each of the two possibilities, $V_{q}=W^{+}, Z^{0}$, two histograms are presented assuming that a jet or the anti-neutrino, respectively, are detected in the bin at azimuth $\phi_{3}$. These histograms should be compared with the solid- and dotted-line histograms presented in Fig. 13a.

In Fig. 20a, we present parton-level asymmetries for the 4 subprocesses in which $V_{l}=Z^{0}$ decays to an $e^{+} e^{-}$pair. In these histograms, the $e^{-}$momentum is used to define the $x-z$ plane, and the quark is detected in the azimuthal bin of size $10^{\circ}$ at angle $\phi_{3}$, and no restrictions other than the standard cuts are placed on the other particles. In Fig. 20b, we present hadron-level asymmetries for the process $p \bar{p} \rightarrow e^{-} e^{+}+2$ jets. The histograms represent 4 different types of bins as defined in the figure. It is interesting that the asymmetries for which $V_{l}=Z^{0}$ are somewhat smaller than those for $V_{l}=W^{-}$presented in Fig. 19b.

\section{CONCLUSIONS}

In this paper we have presented predictions for parity-violating asymmetries that arise from quantum mechanical interference effects between the strong and electroweak components of the Standard Model. The final states in which these asymmetries manifest themselves involve an electroweak boson, either a real photon, or a $W$ or $Z$ observed say in its leptonic decay mode, and a pair of large $p_{T}$ jets with the invariant mass of the pair equal to the mass of a $W$ or a $Z$. These asymmetries might be observable in $p p$ and $p \bar{p}$ collisions above the threshold for production of pairs of electroweak bosons, i.e., at center of mass energies in the $\mathrm{TeV}$ range and these asymmetries are comparable in magnitude to the pair-production cross sections. Unlike the pair production cross section which is parity conserving, the interference contribution is parity-violating, and this might make it easier to observe above a rather formidable QCD background of electroweak boson +2 jet events. Actual observation of these effects will require somewhat higher integrated luminosities than are currently available for example at the Tevatron at Fermilab. The results presented in this paper are therefore offered as examples of the many interesting and subtle Standard Model

predictions that can be studied at a very-high luminosity $\mathrm{TeV}$ hadron collider; they are also offered as examples of an unusual kind of experimental signature involving multi-particle final states that may have analogs in other high-energy collision processes. 


\section{ACKNOWLEDGMENTS}

This work has benefitted from very helpful remarks by and conversations with M. Chanowitz, R.K. Ellis, S.D. Ellis, I. Hinchliffe and D. Sivers, some years ago, and with many other colleagues since. One of us (R.J.G.) would like to acknowledge the hospitality of D. Sivers, C. Zachos and the theory group at Argonne National Laboratory where part of this work was done, as well as the Argonne Division of Educational Programs for a Summer Faculty Participation Award in 1986. He would also like to thank W.A. Bardeen, R.K. Ellis and the Theoretical Physics Department at Fermilab for their kind hospitality in April and May of 1992. This work was supported in part by the National Science Foundation under grant numbers PHY83-10883, PHY87-13231, and PHY92-12177.

\section{APPENDIX A: CONVENTIONS FOR MASSLESS SPINORS}

In this Appendix we define precisely the spinors in terms of which the helicity amplitudes in this paper are presented. We follow for the most part the notation and conventions introduced in various of Refs. [13]. There are some subtle differences between some of these conventions which we discuss for clarity and completeness.

\section{a. Massles fermions and anti-fermions with positive energy}

The (four-component) spinor wave function associated with the annihilation of a massless fermion with momentum $k_{i}$ and helicity $\lambda= \pm \frac{1}{2}$ is a solution of the two equations

$$
\begin{aligned}
& \not k_{i} u_{\lambda}\left(k_{i}\right)=0, \\
& \gamma_{5} u_{\lambda}\left(k_{i}\right)=\operatorname{sign}(\lambda) u_{\lambda}\left(k_{i}\right) .
\end{aligned}
$$

Here, $k^{0}>0$ and $k \cdot k=0$, and we use the $\gamma$ matrix conventions of Bjorken and Drell [22]. The solution of the two equations (A1) is unique up to a multiplicative constant because the first equation relates the two lower components $\chi$ of the spinor $u$ to the upper components $\phi$, and the second then yields an eigenvalue equation for the upper components:

$$
\chi_{\lambda}=\frac{\vec{\sigma} \cdot \vec{k}_{i}}{k_{i}^{0}} \phi_{\lambda}=\operatorname{sign}(\lambda) \phi_{\lambda} .
$$

The multiplicative constant is determined up to an arbitrary phase factor by normalizing the spinor so that

$$
u_{\lambda}\left(k_{i}\right) \bar{u}_{\lambda}\left(k_{i}\right)=\omega_{\operatorname{sign}(\lambda)} \not k_{i} \quad, \quad \omega_{ \pm} \equiv \frac{1 \pm \gamma_{5}}{2}
$$

The fermion spinors have thus been determined up to two arbitrary phase factors, one for each helicity.

The four-component spinor wave function associated with the creation of an anti-fermion with momentum $k_{i}$ and helicity $-\lambda$ is a solution of the two equations 


$$
\begin{aligned}
& \not k_{i} v_{\lambda}\left(k_{i}\right)=0 \\
& \gamma_{5} v_{\lambda}\left(k_{i}\right)=-\operatorname{sign}(\lambda) v_{\lambda}\left(k_{i}\right) .
\end{aligned}
$$

It is convenient and economical to determine the phases of the antifermion spinors as follows:

$$
u_{\lambda}\left(k_{i}\right)=v_{-\lambda}\left(k_{i}\right) \equiv|i, \operatorname{sign}(\lambda)\rangle \text {. }
$$

\section{b. Charge conjugation}

We next fix the relative phase of spinors of opposite helicity using charge conjugation, which is defined as follows:

$$
u \rightarrow u^{c}=C \bar{u}^{T}
$$

where the unitary matrix $C$ has the properties

$$
C^{-1} \gamma_{\mu} C=-\gamma_{\mu}^{T} \quad, \quad C^{-1}=-C^{*}
$$

the first of these properties ensures that charge conjugation reverses the signs of $\not k_{i}$ and $\gamma_{5}$ (i.e. interchanges Eqs. (A1) and (A4)), and the second ensures that $\left(u^{c}\right)^{c}=u$. Given a particular choice of $C$, for example $C=i \gamma^{2} \gamma^{0}$, it follows that

$$
u_{\lambda}^{c}\left(k_{i}\right)=e^{i \eta\left(k_{i}\right)} u_{-\lambda}\left(k_{i}\right),
$$

where the phase constant $\eta\left(k_{i}\right)$ is independent of the helicity $\lambda$.

Refs. 13 propose to fix the phase $\eta\left(k_{i}\right)$ in slightly different ways which, however, lead to the same amplitude expressions, as we shall show. Kleiss and Stirling relate spinors of opposite helicity as follows: Choose a reference momentum $k_{0}$ with $k_{0}^{0}>0$ and $k_{0} \cdot k_{0}=0$ that is not parallel to any other external momentum $k_{i}, i=1,2,3, \ldots$, and an orthogonal space-like unit vector $n_{0}$ with $n_{0} \cdot n_{0}=-1$ and $k_{0} \cdot n_{0}=0$, and define two basic spinors

$$
u_{+\frac{1}{2}}\left(k_{0}\right)=\not h_{0} u_{-\frac{1}{2}}\left(k_{0}\right) \text {. }
$$

These two spinors are normalized as in Eq. (A3) but their phase is not specified. Then, for any other massless spinor with momentum $k_{i}, i=1,2,3, \ldots$,

$$
u_{\lambda}\left(k_{i}\right)=\frac{\not k_{i}}{\sqrt{2 k_{i} \cdot k_{0}}} u_{-\lambda}\left(k_{0}\right) \text {. }
$$

This definition obviously determines the relative phase of spinors of opposite helicity and hence the charge conjugation phase $\eta\left(k_{i}\right)$. It is not difficult to show that in fact

$$
e^{i \eta\left(k_{i}\right)}=-e^{i \eta\left(k_{0}\right)}
$$

i.e., the charge conjugation phase depends only on the reference momentum $k_{0}$ and not on the momentum $k_{i}$ of the fermion. A simple way of showing this is to relate the scalar product $\bar{u}_{\frac{1}{2}}\left(k_{0}\right) u_{\frac{1}{2}}^{c}\left(k_{i}\right)$ to the product $\bar{u}_{\frac{1}{2}}\left(k_{0}\right) u_{-\frac{1}{2}}\left(k_{i}\right)$ using Eqs. (A6), (A7), and (A8). 
$\mathrm{Xu}$ et al., and Gunion and Kunszt [13] fix the relative phase of spinors of opposite helicity by the requirement

$$
u_{\lambda}^{c}\left(k_{i}\right)=u_{-\lambda}\left(k_{i}\right) .
$$

This evidently corresponds to the special choice $\eta\left(k_{0}\right)=\pi$ in the Kleiss-Stirling definition.

To summarize, Xu et al., and Gunion and Kunszt allow a single arbitrary overall phase for each particle of momentum $k_{i}$, while Kleiss and Stirling allow a choice of the reference vectors $k_{0}$ and $n_{0}$ as well as a single arbitrary phase for say the spinor $u_{\frac{1}{2}}\left(k_{0}\right)$. It may be important to take these differences between these two definitions into account when evaluating helicity amplitudes numerically since the different definitions, as well as whatever choices are made in fixing the remaining unspecified overall phases, will affect the sizes of various terms and thus the occurrence of for example numerical errors due to roundoff. However, both defintions lead to the same analytical relations between spinor products that we have used to simplify the analytical results presented in this paper, and which we discuss next.

\section{c. Useful identities involving spinor products}

We next list, for reference, some identities that are extremely useful in simplifying helicity-amplitude expressions.

Spinors can be written in compact bra-ket notation as follows:

$$
\begin{aligned}
& u_{\lambda}\left(k_{i}\right) \equiv\left|i, \sigma_{i}\right\rangle \equiv\left|i \sigma_{i}\right\rangle, \\
& \bar{u}_{\lambda}\left(k_{i}\right) \equiv\left\langle i \sigma_{i}\right|,
\end{aligned}
$$

where $\sigma_{i}=\operatorname{sign}\left(\lambda_{i}\right)$, and we assume that $k^{0}>0$.

Let $\Gamma^{(n)}$ be a string consisting of a product of $n$ Dirac $\gamma$ matrices, and $\Gamma_{R}^{(n)}$ be the string with the matrices written in reverse order. The anti-commutation relation $\left\{\gamma_{\mu}, \gamma_{5}\right\}=0$, together with Eqs. (A1), imply the helicity selection rules

$$
\begin{aligned}
\left\langle i \sigma\left|\Gamma^{(2 n)}\right| j \sigma\right\rangle & =0, \\
\left\langle i \sigma\left|\Gamma^{(2 n+1)}\right| j,-\sigma\right\rangle & =0 .
\end{aligned}
$$

The phase choice implied in Eq. (A12), together with the the behavior of $\gamma$ matrices under charge conjugation, Eq. (A7), imply the following "charge conjugation identity":

$$
\left\langle i \sigma_{i}\left|\Gamma^{(n)}\right| j \sigma_{j}\right\rangle=(-1)^{(n+1)}\left\langle j,-\sigma_{j}\left|\Gamma_{R}^{(n)}\right| i,-\sigma_{i}\right\rangle \text {. }
$$

This identity is in fact also obeyed by spinors with phases chosen according to the prescription of Kleiss and Stirling, Eq. (A10), since their charge conjugation phase is independent of momentum. (Kleiss and Striling refer to Eq. (A15) as the "line reversal identity", and prove it without appealing directly to charge conjugation).

An extremely useful identity involving $\gamma$ matrices with their indices contracted is the Chisholm identity:

$$
\left\langle i \sigma\left|\gamma_{\mu}\right| j \sigma\right\rangle \gamma^{\mu}=2[|j \sigma\rangle\langle i \sigma|+| i,-\sigma\rangle\langle j,-\sigma|]
$$


Proofs of this identity can be found in Refs. 13.

Another very useful identity is the "cyclic identity":

$$
|i \pm\rangle\langle j \mp \mid k \pm\rangle+|j \pm\rangle\langle k \mp \mid i \pm\rangle+|k \pm\rangle\langle i \mp \mid j \pm\rangle=0
$$

A proof of this identity can be found in Xu et al., [13]. We sketch here an alternate simple proof which uses the Kleiss-Stirling spinor definitions. Using Eq. (A10), we can write

$$
\begin{aligned}
|i-\rangle & \langle j+|-| j-\rangle\langle i+| \\
& =\frac{1}{\sqrt{4 k_{i} \cdot k_{0} k_{j} \cdot k_{0}}}\left(\not k_{i} \not k_{0} \not k_{0} \not k_{j}-\not k_{i} \not k_{0} \not k_{0} \not k_{j}\right) \omega_{-} \\
& =a+b \gamma_{5}+c^{\mu \nu} \sigma_{\mu \nu}
\end{aligned}
$$

The last line expresses the most general form of a product of an even number of $\gamma$ matrices. The coefficients can be found by forming the appropriate traces. It is easy to show that $a=-b=\frac{1}{2}\langle j+\mid i-\rangle$, and the fact that $c^{\mu \nu}=0$ is easily proven using the charge conjugation identity Eq. (A15). The cyclic identity (A17) with the lower signs follows when Eq. (A18) is multiplied on the right by $|k-\rangle$. The cyclic identity with the upper signs follows from charge conjugation. The cyclic identity is particularly useful in simplifying helicity amplitudes that involve a non-abelian three-vector-boson vertex.

\section{d. Negative energies and crossing}

If all external particles in a "generic diagram" are taken to be outgoing, momentum conservation implies that some of these particles must have negative energies. It is evident, for example from the square root factor in Eq. (A10), that reversing the sign of the energy is a discontinuous transformation and can introduce and ambiguity in the phase of a spinor. Gunion and Kunszt propose to fix this ambiguity by requiring that a change in the sign of the momentum of a particle in a helicity amplitude is equivalent to crossing that particle between initial and final states. It is easy to see that this might be accomplished by defining

$$
\begin{aligned}
& |-k, \sigma\rangle=i|k \sigma\rangle, \quad \text { for } k^{0}>0 . \\
& \langle-k, \sigma|=i\langle k \sigma|,
\end{aligned}
$$

An immediate consequence of this choice is that the spinor normalization (A3) and the identities (A14 A17) preserve their forms when the sign of any of the momenta $k_{i}$ involved is reversed. Conversely, demanding that these identities be form invariant under this sign change determines the choice of phases in (A19) up to a sign. In this sense, the phase choice is implicit in the definitions of Kleiss and Stirling. The choice of phases in Eq. (A19) requires that we modify the definition of bra spinors, introduced in Eq. (A13) for positive energy, as follows:

$$
\langle k \sigma| \equiv \operatorname{sign}\left(k^{0}\right) \bar{u}_{\lambda}(k)=\operatorname{sign}\left(k^{0}\right) u_{\lambda}^{*}(k)^{T} \gamma_{0} \equiv u_{\lambda}^{+}(k)^{T} \gamma_{0} .
$$

Following Ref. [15], we have introduced a "+ conjugation" operation $\left(^{+}\right)$which is the usual complex conjugation $\left(^{*}\right)$ operation, but followed by a change of sign when it acts on a 
spinor with negative energy. Note that complex conjugation is also to be replaced by "+ conjugation" in the definition of charge conjugation in Eq. (A6). Most importantly, the process of "squaring" an amplitude $\mathcal{A}$ to obtain a probability (cross section) is done by multiplying the amplitude by its "+ conjugate" rather than its complex conjugate. The following elementary example illustrates that this leads to a crossing-invariant result:

$$
\begin{aligned}
& \left\langle k_{1} \sigma \mid k_{2},-\sigma\right\rangle\left\langle k_{1} \sigma \mid k_{2},-\sigma\right\rangle^{+} \\
& \quad=\operatorname{sign}\left(k_{1}^{0} k_{2}^{0}\right)\left|\left\langle\operatorname{sign}\left(k_{1}^{0}\right) k_{1}, \sigma \mid \operatorname{sign}\left(k_{1}^{0}\right) k_{2},-\sigma\right\rangle\right|^{2} \\
& \quad=2 k_{1} \cdot k_{2} .
\end{aligned}
$$

In a practical computation, the following relation is useful in forming a probability:

$$
\left\langle i \sigma_{i}|\Gamma| j \sigma_{j}\right\rangle^{+}=\left\langle j \sigma_{j}\left|\Gamma_{R}\right| i \sigma_{i}\right\rangle \text {. }
$$

Here $\Gamma$ is a product of $\gamma$ matrices, and $\Gamma_{R}$ the product with the matrices taken in reverse order. This relation is valid for positive and negative energies, and, when combined with the completeness relation (A3), is also seen to produce crossing invariant probabilities.

\section{e. Photon wave functions}

To compute the helicity amplitudes for production of a real photon plus 2 jets, we need to specify the wave function of a photon with definite helicity $\lambda= \pm 1$. If the momentum $k$ of the photon is given, its wave function is only determined up to a gauge transformation and an arbitrary multiplicative constant. We shall follow Xu et al., [13] to define the phase and gauge of the wave function in terms of spinors associated with $k$ and with a momentum $p$ chosen such that $k \cdot p \neq 0, p \cdot p=0$ and $p^{0}>0$, as follows:

$$
\epsilon_{\lambda}^{\mu}(k ; p)=\lambda \frac{\left\langle p,-\lambda\left|\gamma^{\mu}\right| k,-\lambda\right\rangle}{\sqrt{2}\langle p \lambda \mid k,-\lambda\rangle} .
$$

If the spinors are normalized and their phase defined as in the earlier in this Appendix, it is easy to verify that the photon wave functions satisfy the following conditions:

$$
\begin{aligned}
\epsilon_{\lambda}^{\mu}(k ; p) & =\epsilon_{\lambda}^{*}(k ; p), \\
k \cdot \epsilon_{\lambda}(k ; p) & =0 \\
\epsilon_{\lambda}(k ; p) \cdot \epsilon_{\lambda^{\prime}}^{*}\left(k ; p^{\prime}\right) & =-\delta_{\lambda, \lambda^{\prime}}, \\
\epsilon_{\lambda}^{\mu}\left(k ; p^{\prime}\right) & =\epsilon_{\lambda}^{\mu}(k ; p)+\frac{\sqrt{2} \lambda\left\langle p^{\prime},-\lambda \mid p \lambda\right\rangle}{\left\langle p^{\prime},-\lambda \mid k \lambda\right\rangle\langle k,-\lambda \mid p \lambda\rangle} k^{\mu} .
\end{aligned}
$$

In practice, the auxiliary momentum $p$ is chosen to simplify a given helicity amplitude as much as possible. The last of Eqs. (A24) shows that any two choices of $p$ yield polarization vectors that differ by a change of gauge: thus the same auxiliary momentum must be used in all members of a gauge invariant set of diagrams.

We note finally that $\epsilon_{\lambda}^{\mu}(k ; p)$ is the wave function of an outgoing photon, i.e., it is the polarization vector associated with the creation operator in the photon field operator. To 
verify that $\lambda$ as defined in Eq. (A23) corresponds to the correct circular polarization, let $n_{1}$ and $n_{2}$ be two linear polarization vectors which satisfy

$$
n_{1}^{2}=n_{2}^{2}=-1, \quad n_{i} \cdot k=0, \quad \vec{n}_{1} \times \vec{n}_{2}=\vec{k}
$$

Then, the following phase relation between the transverse components

$$
n_{2} \cdot \epsilon_{\lambda}(k ; p)=i \lambda n_{2} \cdot \epsilon_{\lambda}(k ; p)
$$

shows that $\lambda= \pm 1$ correspond to right and left circular polarization respectively. wave function of an incoming photon is obtained either by crossing $k \rightarrow-k$ or by complex conjugation:

$$
\epsilon_{\lambda}(-k ; p)=\epsilon_{\lambda}^{*}(k ; p)
$$

\section{APPENDIX B: PROPERTIES OF INVARIANT FUNCTIONS}

In this Appendix, we collect and discuss several useful formulas involving the various functions in terms of which the analytic results of this paper have been presented.

\section{a. Properties of $F_{123456}$}

This function was defined in Eq. (2.9). Using $\langle i \mid j\rangle=-\langle j \mid i\rangle$, see Eq. (A15), we see that

$$
F_{123456}=-F_{321456}=-F_{163452} .
$$

Using the cyclic identity Eq. (A17), we see that

$$
F_{123456}+F_{143652}+F_{163254}=0 \text {. }
$$

Finally, if the six momenta $k_{i}$ satisfy $\sum_{i=1}^{6} k_{i}=0$, it is easy to show using Eq. (A3) that

$$
F_{123456}=-F_{216543}^{+} .
$$

\footnotetext{
${ }^{6}$ This relation is easily verified by direct computation e.g., by using a frame and gauge in which $k=k^{0}(1,0,0,1)$ and $p=p^{0}(1,1,0,0)$.
} 


\section{b. Triple-boson vertex contribution}

As an application of some of the identites listed in Appendix A we show that the contribution of the diagram in Fig 5 with the non-abelian triple-boson vertex can be expressed in terms of the $F$ functions. This rather remarkable result is presented without a derivation in [15]; since the derivation appears to be non-trivial, we include it here for completeness. It is not difficult to see that the amplitude corresponding to this diagram, with all helicities chosen to be negative for example, has the following Lorentz factor:

$$
\begin{aligned}
& \left\langle 1-\left|\gamma^{\lambda}\right| 2-\right\rangle\left\langle 3-\left|\gamma^{\mu}\right| 4-\right\rangle\left\langle 5-\left|\gamma^{\nu}\right| 6-\right\rangle \\
& \times\left[g_{\nu \lambda}\left(k_{12}-k_{56}\right)_{\mu}+g_{\lambda \mu}\left(k_{34}-k_{12}\right)_{\nu}+g_{\mu \nu}\left(k_{56}-k_{34}\right)_{\lambda}\right] \\
& =4\langle 1-\mid 5+\rangle\langle 6+\mid 2-\rangle[\langle 1-\mid 3+\rangle\langle 4+\mid 1-\rangle+\langle 2-\mid 3+\rangle\langle 4+\mid 2-\rangle] \\
& -4\langle 1-\mid 3+\rangle\langle 4+\mid 2-\rangle[\langle 1-\mid 5+\rangle\langle 6+\mid 1-\rangle+\langle 2-\mid 5+\rangle\langle 6+\mid 2-\rangle] \\
& -4\langle 3-\mid 5+\rangle\langle 6+\mid 4-\rangle[\langle 1-\mid 3+\rangle\langle 3+\mid 2-\rangle+\langle 1-\mid 4+\rangle\langle 4+\mid 2-\rangle] .
\end{aligned}
$$

To obtain the right hand side of this equation, we have used the Chisholm identity (A16) as well as Eqs. (A1-A3) and (A15). While the right hand side is not obviously expressible in terms of the $F$ functions, it is actually equal to the difference $\left(F_{123456}-F_{125634}\right)$, as can be seen by examining the following expression:

$$
\begin{aligned}
& \text { R.H.S. }-\left(F_{123456}-F_{125634}\right) \\
& \begin{aligned}
= & \{\langle 3-\mid 5+\rangle\langle 4+\mid 2-\rangle[\langle 1-\mid 4+\rangle\langle 4+\mid 6-\rangle+\langle 1-\mid 5+\rangle\langle 5+\mid 6-\rangle] \\
& -\langle 3-\mid 5+\rangle\langle 1-\mid 3+\rangle[\langle 6+\mid 4-\rangle\langle 3+\mid 2-\rangle+\langle 6+\mid 2-\rangle\langle 4+\mid 3-\rangle] \\
& -\langle 4+\mid 2-\rangle\langle 6+\mid 2-\rangle[\langle 1-\mid 3+\rangle\langle 2-\mid 5+\rangle+\langle 1-\mid 5+\rangle\langle 3-\mid 2+\rangle]\} .
\end{aligned}
\end{aligned}
$$

Using the cyclic identity (A17) on the terms in each of the second ane third square brackets

on the right hand side of the above equation, it is easy to see that the right hand side of the above equation can be written

$$
\begin{aligned}
& 4\langle 3-\mid 5+\rangle\langle 4+\mid 2-\rangle[\langle 1-\mid 2+\rangle\langle 2+\mid 6-\rangle \\
& \quad+\langle 1-\mid 3+\rangle\langle 3+\mid 6-\rangle+\langle 1-\mid 4+\rangle\langle 4+\mid 6-\rangle+\langle 1-\mid 5+\rangle\langle 5+\mid 6-\rangle],
\end{aligned}
$$

which can be seen to vanish by using Eq. (A3) and momentum conservation.

\section{c. Properties of $(I, J, K)_{123456}$}

These functions were defined in Eqs. (2.52)-(2.53). Using the properties of the functions $F$, see Eqs. (B1)-(B3) it is easy to show that

$$
I_{123456}=I_{123456}^{+}=I_{321456}=I_{163452}=I_{216543} .
$$

Since $I$ is real, it does not contribute to the asymmetries presented in this paper. Nevertheless, we give here an expression for $I$ in terms of invariants for completeness:

$$
I_{123456}=16 s_{13} s_{26}\left[\left(s_{14}+s_{34}\right)\left(s_{15}+s_{35}\right)-s_{13} s_{45}\right] .
$$


The function $J$ can likewise be shown to satisfy

$$
J_{123456}=J_{125634}^{+}=J_{214365} .
$$

Various equivalent expressions for $J$ in terms of invariants can be derived by using Eqs. (B1)-(B3). One such expression can be obtained by writing out Eq. (2.53) using (2.9), and rewriting the various terms using the completeness relation (A3):

$$
\frac{1}{16} J_{123456}=-s_{13} s_{15} s_{24} s_{26}-s_{135} \operatorname{Tr}\left[\gamma_{R} \not k_{6} \not k_{2} \not k_{4} \not k_{3} \not k_{1} \not k_{5}\right] .
$$

The trace in this equation can be expressed in many equivalent forms, one of which is

$$
\begin{aligned}
& \operatorname{Tr}\left[\gamma_{R} \not k_{6} \not k_{2} \not k_{4} \not \not_{3} \not k_{1} \not k_{5}\right]=s_{15} T_{2436}-s_{25} T_{1346}-s_{35} T_{1426}+s_{45} T_{1326}+s_{56} T_{1342} \\
& +i\left[s_{13} x_{2456}-s_{15} x_{2346}-s_{35} x_{1246}-s_{24} x_{1356}+s_{26} x_{1345}+s_{46} x_{1235}\right]
\end{aligned}
$$

where

$$
T_{1234} \equiv \operatorname{Tr}\left[\not \not_{1} \not K_{2} \not \not Z_{3} \not K_{4}\right]=s_{12} s_{34}-s_{13} s_{24}+s_{14} s_{23} .
$$

The real part of $J$ in Eq. (B11) was given in Ref. [15], which however did not discuss the imaginary part. A slightly more compact and convenient expression for $J$ can be obtained by using Eq. (B3):

$$
\begin{aligned}
& \frac{1}{16} J_{1234556}=-F_{123456} F_{214365} \\
& \quad=-s_{13} s_{24} \operatorname{Tr}\left[\gamma_{R} \not k_{3} \not k_{6} \not k_{4} \not k_{5}\right]+s_{13}\left(s_{26}+s_{46}\right) \operatorname{Tr}\left[\gamma_{R} \not k_{3} \not k_{2} \not k_{4} \not k_{5}\right] \\
& \quad+\left(s_{15}+s_{35}\right) s_{24} \operatorname{Tr}\left[\gamma_{R} \not k_{3} \not k_{6} \not k_{4} \not k_{1}\right]-\left(s_{15}+s_{35}\right)\left(s_{26}+s_{46}\right) \operatorname{Tr}\left[\gamma_{R} \not \not_{3} \not k_{2} \not k_{4} \not k_{1}\right] .
\end{aligned}
$$

This yields the following expression for the imaginary part of $J$ :

$$
\begin{aligned}
& \operatorname{Im} J_{123456}=32\left[s_{13} s_{24} x_{3456}+s_{13}\left(s_{26}+s_{46}\right) x_{2345}\right. \\
& \left.\quad-\left(s_{15}+s_{35}\right) s_{24} x_{1346}-\left(s_{15}+s_{35}\right)\left(s_{26}+s_{46}\right) x_{1234}\right] .
\end{aligned}
$$

The function $K$ satisfies

$$
K_{123456}=K_{321456} .
$$

Various equivalent expressions for $K$ can be derived, one of the simplest of which is got by writing $F$ in terms of $F^{+}$:

$$
\begin{aligned}
K_{123456} & =F_{216543}^{+} F_{436521} \\
& =16 s_{13}\left\{s_{246} \operatorname{Tr}\left[\gamma_{R} \not K_{6} \not K_{2} \not K_{5} \not K_{4}\right]-s_{26} s_{46}\left(s_{25}+s_{45}+s_{56}\right)\right\},
\end{aligned}
$$

which yields

$$
\operatorname{Im} K_{123456}=32 s_{13} s_{246} x_{2456} .
$$

The expressions for the imaginary parts of $X_{123456}$ and $Y_{123456}$ given in Eqs. (2.62,2.63) follow from the definitions in Eqs. (2.55, 2.56) and from Eqs. (B14) and (B17). 


\section{d. Properties of $\left(H, X^{\gamma}, Y^{\gamma}\right)_{12345}$}

These functions were introduced in Eqs. (2.34) and (2.86-2.89). Since they involve five momenta which obey $\sum_{i=1}^{5} k_{i}=0$, they are much simpler than the functions $I, J, K, X, Y$. It is straightforward to show that

$$
\begin{aligned}
& X_{12345}^{\gamma}=\frac{8 s_{13}^{2} \operatorname{Tr}\left[\gamma_{R} \not k_{1} \not \not k_{4} \not \not k_{3} \not \not k_{5}\right.}{s_{14} s_{15} s_{25} s_{35}}, \\
& Y_{12345}^{\gamma}=\frac{8 s_{13}^{2} \operatorname{Tr}\left[\begin{array}{lllll}
\gamma_{R} & \not k_{2} & \not k_{3} & \not k_{4} & \not k_{5}
\end{array}\right]}{s_{15} s_{23} s_{25} s_{45}} \text {, }
\end{aligned}
$$

from which the imaginary parts can easily be extracted. 


\section{REFERENCES}

[1] W. A. Bardeen, H. Fritzsch, and M. Gell-Mann, in Scale and Conformal Symmetries in Hadron Physics, edited by R. Gatto (Wiley, New York, 1973); D. J. Gross and F. Wilczek, Phys. Rev. Lett. 30, 1343 (1973); H. D. Politzer, ibid. 1343; G. 'tHooft (unpublished); K. G. Wilson, Phys. Rev. D 102445 (1974).

[2] S. L. Glashow, Nucl. Phys. B22, 579 (1961); A. Salam and J. C. Ward, Phys. Lett. 13, 168 (1964); S. Weinberg, Phys. Rev. Lett. 19, 1264 (1967).

[3] Ya. B. Zel'dovich, Zh. Eksp. Teor. Fiz. 9, 964 (1959) [Sov. Phys. JETP 36, 682 (1959)].

[4] B. Adeva at al., Phys. Rev. Lett. 48, 1701 (1982); W. Bartel at al., Phys. Lett. 108B, 140 (1982); H.-J. Behrend at al., Z. Phys. C 14, 283 (1982); R. Brandelik at al., Phys. Lett. 110B, 172 (1982); E. Fernandez at al., Phys. Rev. Lett. 50, 1238 (1983); M. E. Levi at al., Phys. Rev. Lett. 51, 1941 (1983).

[5] C.Y. Prescott at al., Phys. Lett. 77B, 347 (1978).

[6] P. E. G. Baird at al., Phys. Rev. Lett. 39, 798 (1977); L. L. Lewis at al., Phys. Rev. Lett. 39, 795 (1977); L. M. Barkov and M. S. Zolotorev, Pis'ma Zh. Eksp. Teor. Fiz. 27, 379 (1978) [JETP Lett. 27, 357 (1979)]; M. A. Bouchiat at al., Phys. Lett. 117B, 358 (1982).

[7] E. M. Henley and F. R. Krejs, Phys. Rev. D 11, 605 (1975); K. H. Craig, Nucl. Phys. B109, 156 (1976); J. Missimer, L. Wolfenstein, and J. Gunion, Nucl. Phys. B111, 20 (1976); E. Fishbach and G. W. Look, Phys. Rev. D 13, 752 (1976); L. L. Frankfurt and V. B. Kopeliovich, Nucl. Phys. B103, 360 (1976); M. Abud, R. Gatto, and C. A. Savoy, Ann. Phys. (NY), 122, 219 (1979); H. Y. Cheng and E. Fischbach, Phys. Rev. D 19, 2123 (1979), Erratum ibid. D 20, 1247 (1979); J. Ranft and G. Ranft, Nucl. Phys. B165, 395 (1980); U. Baur, E. W. N. Glover, and A. D. Martin, Phys. Lett. 232B, 519 (1989).

[8] R. J. Gonsalves, Phys. Rev. Lett. 56, 1647 (1986).

[9] L. Clavelli and G. v. Gehlen, Phys. Rev. D 27, 1495 (1983).

[10] H.K.K. Tung and C.F. Wai, Academia Sinica Preprint IP-ASTP-4-93, 1993 (to be published).

[11] C. F. Wai, Academia Sinica Preprint IP-ASTP-14-92, 1992 (to be published).

[12] C. F. Wai, Ph.D thesis, State University of New York at Buffalo, 1988, UMI 88-12397-mc (microfiche).

[13] P. De Causmaecker, R. Gastmans, W. Troost, and T.T. Wu, Nucl. Phys. B206, 53 (1982); F. A. Berends at al., Nucl. Phys. B206, 61 (1982); ibid., B264, 265 (1986), and references therein; Z. Xu, D.-H. Zhang, and L. Chang, Tsinghua Preprints TUTP-84/4,5,6; Nucl. Phys. B291, 392 (1987); J. F. Gunion and Z. Kunszt, Phys. Lett. 161B, 333 (1985); R. Kleiss and W. J. Stirling, Nucl. Phys. B262, 167 (1985); R. Gastmans and T. T. Wu, The Ubiquitous Photon: Helicity Method for QED and QCD (Oxford University Press, 1990).

[14] S. D. Ellis, R. Kleiss, and W. J. Stirling, Phys. Lett. 154B, 435 (1985); J. F. Gunion and Z. Kunszt, Phys. Lett. 161B, 333 (1985); R. Kleiss and W. J. Stirling, Nucl. Phys. B262, 235 (1985); M. Mangano and S. Parke, Phys. Rev. D 41, 59 (1990).

[15] J. F. Gunion and Z. Kunszt, Phys. Rev. D 33, 665 (1986).

[16] R. J. Eden, P. V. Landshoff, D. I. Olive, and J. C. Polkinghorne, The Analytic S-matrix (Cambridge University Press, 1966).

[17] A. D. Martin, R. G. Roberts, and W. J. Stirling, Phys. Rev. D 371161 (1988); Phys. Lett. 206B, 327 (1988); Mod. Phys. Lett. A 4, 1135 (1989). 
[18] G. P. Lepage, J. Comput. Phys. 27, 192 (1978).

[19] W.J. Stirling, R. Kleiss, and S.D. Ellis, Phys. Lett. 163B, 261 (1985).

[20] R. K. Ellis and R. J. Gonsalves, in Supercollider Physics: Proceedings of the Oregon Workshop on Super High Energy Physics, Eugene, Oregon, 1985, edited by D. E. Soper (World Scientific, Singapore, 1986), p. 287.

[21] CDF Collaboration (F. Abe, et al.), FERMILAB-PUB-93-063-E, April 1993, submitted to Phys. Rev. Lett.

[22] J.D. Bjorken and S.D. Drell, Relativistic Quantum Fields, (McGraw-Hill, New York, 1965). 


\section{FIGURES}

FIG. 1. Chromo-electroweak interference. A solid line represents a quark or antiquark with color $c$ and flavor $f$. The wavy line represents a color-neutral electroweak boson and the curly line a flavor-neutral gluon. The two amplitudes can interfere because their initial states (on the left) can have identical quantum numbers as can their final states (on the right).

FIG. 2. Hadrons $h_{1}$ and $h_{2}$ collide to produce an on-shell electroweak boson $V$ and two hadron jets $j_{1}$ and $j_{2}$ at large transverse momentum.

FIG. 3. Generic diagrams with one electroweak boson (wavy line) and four partons in the final state. Choosing two partons in all possible ways, and crossing them to the initial state generates all tree-level contributions to the processes in Eq. (2.1).

FIG. 4. These generic "electroweak" diagrams can interfere with the "QCD" diagrams of Fig. 3a.

FIG. 5. The dominant diagrams which contribute to chromo-electroweak interference. $V_{l} \rightarrow l\left(k_{5}\right)+\bar{l}\left(k_{6}\right)$ with $\left(k_{5}+k_{6}\right)^{2}=M_{V_{l}}^{2}$, and $V_{q} \rightarrow q\left(k_{3}\right)+\bar{q}\left(k_{4}\right)$ with $\left(k_{3}+k_{4}\right)^{2}=M_{V_{q}}^{2}$. When crossed to the initial state, $q\left(k_{1}\right)$ and $\bar{q}\left(k_{2}\right)$ represent an incoming antiquark with momentum $p_{1}=-k_{2}$ and an incoming antiquark with momentum $p_{2}=-k_{1}$ respectively. Other assignments of particles to the initial state yield amplitudes in which $V_{q}$ is far off-shell.

FIG. 6. (a) Momenta in the $x-z$ plane. $p_{1}$ is the momentum of the quark (parton-level asymmetry), the proton ( $p \bar{p}$ collisions), or one of the protons chosen arbitrarily ( $p p$ collisions). $p_{5}$ is the momentum of $V=W^{ \pm}, Z^{0}$ (3-particle final state) or of the lepton (4-particle final state). A right-handed coordinate system is defined such that $p_{5}^{x}>0$ and $p_{5}^{y}=0$. (b) Momenta in the $x-y$ plane. $p_{3}$ and $p_{4}$ are the momenta of the two jet partons. Parity is violated if the event shown on the left and its mirror image shown on the right occur with different probabilities. In the case of the 4-particle final state, the azimuthal angle $\phi_{6}$ of the antilepton may also be used to define a bin.

FIG. 7. Differential parton-level asymmetries in the process $q \bar{q} \rightarrow W^{-} q \bar{q}$. (a) Contributions from 2 subprocesses with $V_{q}=W^{+}$and 2 with $V_{q}=Z^{0} . \phi_{3}$ is the azimuthal angle of the quark in the final state. (b) Contributions from subprocesses with $V_{q}=Z^{0}$ decaying to a $q \bar{q}$ with flavor $f_{3}$, and 4 different assumptions concerning the distinguishability of the parton observed at azimuth $\phi_{3}$.

FIG. 8. Differential asymmetries of the type in Fig. 7la, for (a) $W+$ production, and (b) $Z^{0}$ production.

FIG. 9. Differential asymmetries of the type in Fig. đa $\mathrm{a}$, for real photon production (a) at $\sqrt{s_{12}}=110 \mathrm{GeV}$ just above threshold for the process, and (b) at $\sqrt{s_{12}}=250 \mathrm{GeV}$. 
FIG. 10. Parton- and hadron-level maximum asymmetries as defined in Eq. (3.4) in $W^{-}$production for (a) $V_{q}=W^{+}$and (b) $V_{q}=Z^{0}$. The parton level asymmetries shown illustrate various assumptions concerning the distinguishability of the final state quark jets. The hadron-level asymmetries are "flavor-blind".

FIG. 11. Maximum asymmetries in $Z^{0}$ production. (a) $V_{q}=W^{ \pm}$. At the parton level, asymmetries for $u \bar{d} \rightarrow u \bar{d} Z^{0}$, i.e., for $V_{q}=W^{-}$, are the same as for $d \bar{u} \rightarrow d \bar{u} Z^{0}$, and are not shown. Both subprocesses are included at the hadron-level since $W^{ \pm}$have the same mass. (b) $V_{q}=Z^{0}$.

FIG. 12. Maximum asymmetries in real photon production. (a) $V_{q}=W^{ \pm}$. At the parton level, asymmetries for $u \bar{d} \rightarrow u \bar{d} Z^{0}$, i.e., for $V_{q}=W^{-}$, are the same as for $d \bar{u} \rightarrow d \bar{u} Z^{0}$, and are not shown. Both subprocesses are included at the hadron-level since $W^{ \pm}$have the same mass. (b) $V_{q}=Z^{0}$.

FIG. 13. Binned asymmetries in $p \bar{p}$ collisions at $2 \mathrm{TeV}$ (a) in $W^{-}$and $Z^{0}$ production for bins of width $500 \mathrm{GeV}$ in the subprocess invariant mass $\sqrt{s_{12}}$, and (b) in real photon production for two different choices of bins in $\sqrt{s_{12}}$. The notation $[\times 10]$ indicates that the histogram has been multiplied by a factor of 10 to show it on the same scale as the others. Note that the algebraic sum of $a^{\mathrm{pv}}$ values in $\phi_{3}$ bins of any each histogram is zero within numerical uncertainties.

FIG. 14. Binned asymmetries for $p p \rightarrow \gamma+2$ jets at $40 \mathrm{TeV}$ with (a) $V_{q}=W^{ \pm}$and (b) $V_{q}=Z^{0}$. The subprocess energy is restricted to the bin $80 \mathrm{GeV}<\sqrt{s_{12}}<280 \mathrm{GeV}$. The rapidities $y_{3}$ and $y_{q}$ of the jet at azimuth $\phi_{3}$ and the photon are further restricted as indicated to produce a non-vanishing asymmetry.

FIG. 15. Binned asymmetries at $40 \mathrm{TeV}$ for (a) $p p \rightarrow W^{-}+2$ jets, and (b) $p p \rightarrow Z^{0}+2$ jets. The subprocess energy is restricted to the bin $150 \mathrm{GeV}<\sqrt{s_{12}}<350 \mathrm{GeV}$. Rapidities $y_{3}$ and $y_{q}$ are restricted as shown to produce non-zero asymmetries.

FIG. 16. Comparison of signal to background in $W^{-}+2$ jet production. (a) Binned cross sections with $200 \mathrm{GeV}<\sqrt{s_{12}}<600 \mathrm{GeV}$. The parity-violating asymmetries have been multiplied by the factors (100 and 1000) indicated to show them on the same scale as the QCD background. Since the background histograms in the regions $s_{34} \approx M_{W}^{2}$ and $s_{34} \approx M_{Z}^{2}$ are almost identical, only one of them is shown. (b) Variation of the signal to background ratio in $p p$ and $p \bar{p}$ collisions as a function of the colliding beam energy.

FIG. 17. Comparison of signal to background in $Z^{0}+2$ jet production. (a) Binned cross sections with $200 \mathrm{GeV}<\sqrt{s_{12}}<600 \mathrm{GeV}$. (b) Variation of the signal to background ratio in $p p$ and $p \bar{p}$ collisions as a function of the colliding beam energy. 
FIG. 18. Comparison of signal to background in real photon +2 jet production. (a) Binned cross sections with $100 \mathrm{GeV}<\sqrt{s_{12}}<500 \mathrm{GeV}$. (b) Variation of the signal to background ratio in $p p$ and $p \bar{p}$ collisions as a function of the colliding beam energy.

FIG. 19. Asymmetries from 4-particle final states. (a) At the parton level for the process $u \bar{u} \rightarrow\left(W^{-} \rightarrow e^{-} \bar{\nu}\right) u \bar{d}$. The $e^{-}$momentum defines the direction $\phi=0$. The dotted histogram is for observation of the $\bar{\nu}$ at azimuth $\phi_{3}$, and the other four histograms for the $u$ quark at azimuth $\phi_{3}$ and with various cuts as indicated on the azimuth $\phi_{4}$ of the $\bar{d}$. (b) At the hadron level for the process $p \bar{p} \rightarrow\left(W^{-} \rightarrow e^{-} \bar{\nu}\right)+2$ jets. For each of the two possibilities for $V_{q}=W^{+}, Z^{0}$, two histograms are shown assuming either the $\bar{\nu}$ or one of the jets observed at azimuth $\phi_{3}$.

FIG. 20. Asymmetries from 4-particle final states. (a) Contributions from the four different subprocess which produce a pair of $Z^{0}$ s, one of which decays to leptons and the other to quarks. The $e^{-}$momentum defines azimuth $\phi=0$, and the quark is observed at azimuth $\phi_{3}$. (b) Contributions from the process $p \bar{p} \rightarrow\left(Z^{0} \rightarrow e^{-} e^{+}\right)+2$ jets. Two histograms each for $V_{q}=W^{ \pm}, Z^{0}$ are shown. The dotted-line histogram assumes the $e^{+}$is detected at azimuth $\phi_{3}$. The other three histograms assume that one jet is detected at azimuth $\phi_{3}$, and the azimuth $\phi_{4}$ of the other jet is unrestricted (solid line), or restriced as indicated (dashed and dot-dashed lines). 

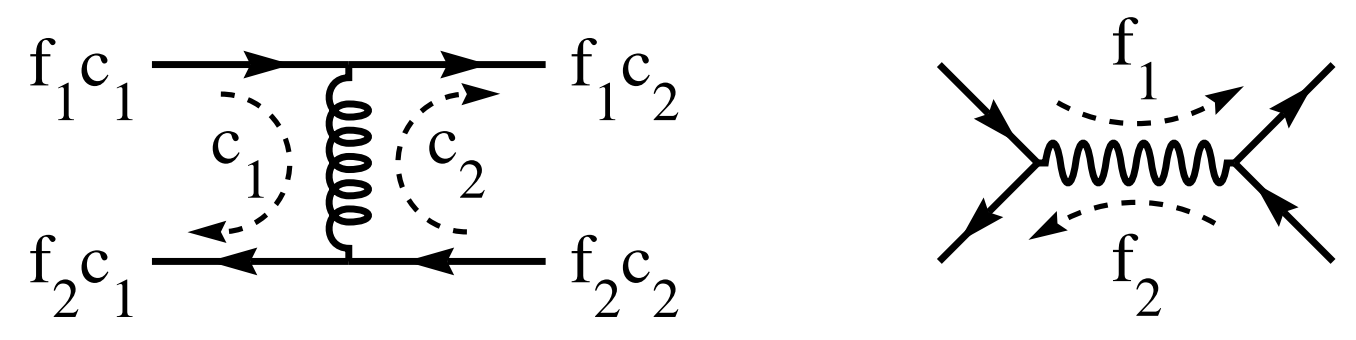

Fig. 1 


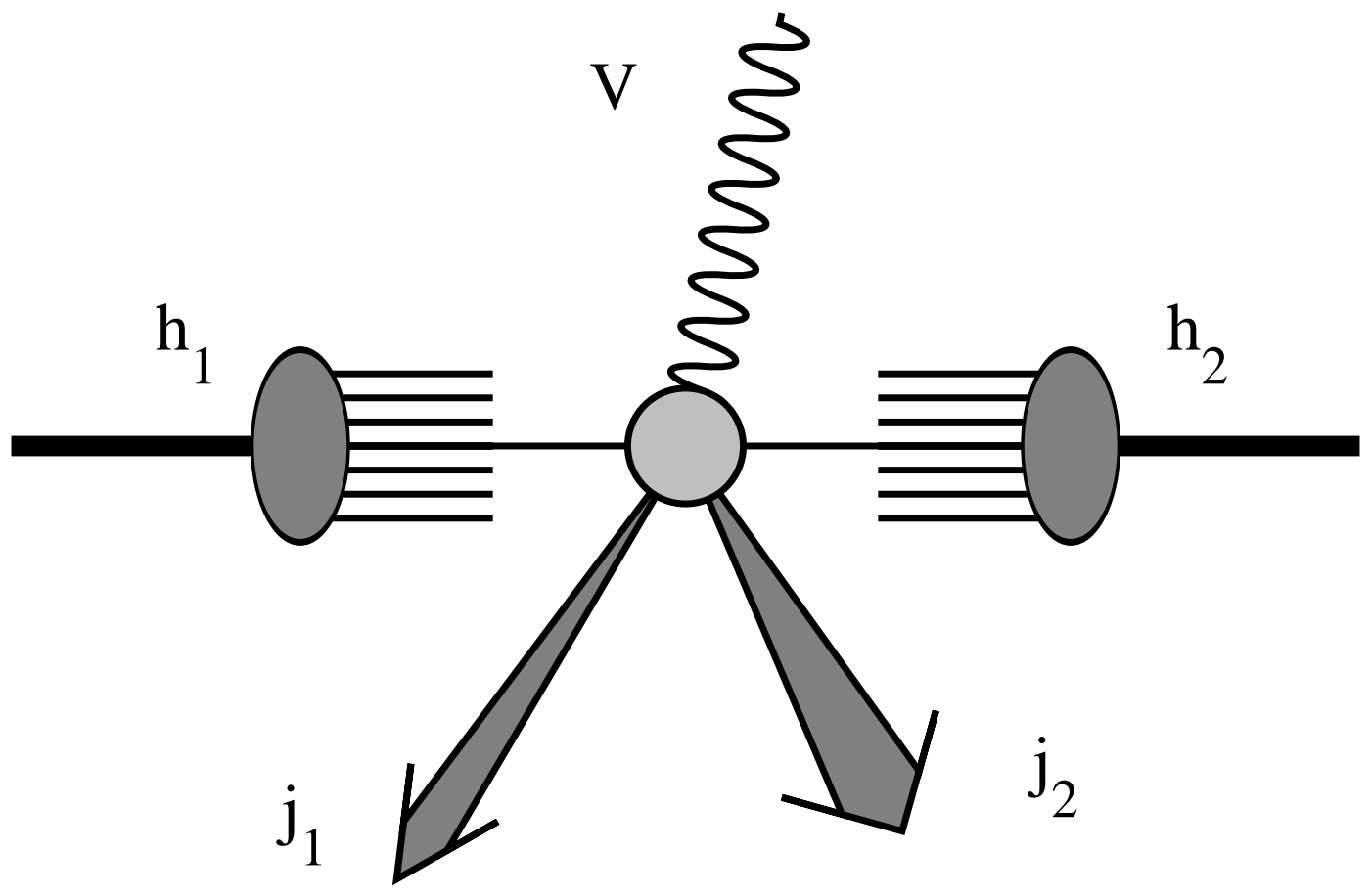

Fig. 2 


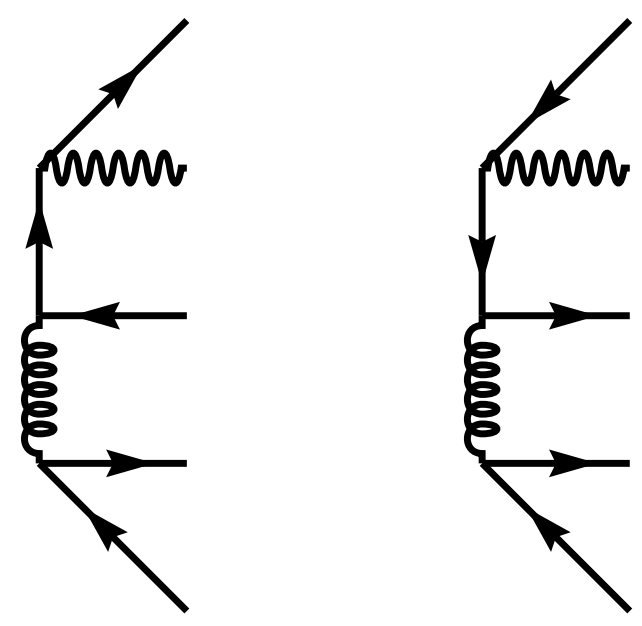

(a)
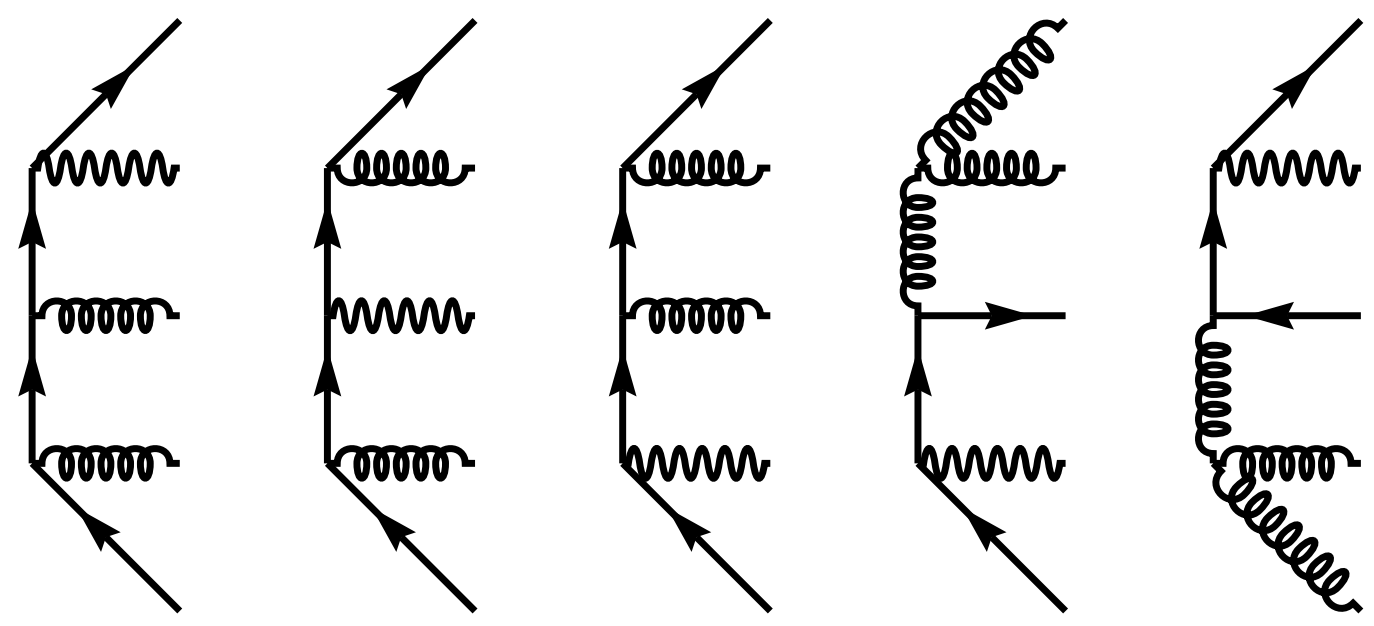

(b)

Fig. 3 

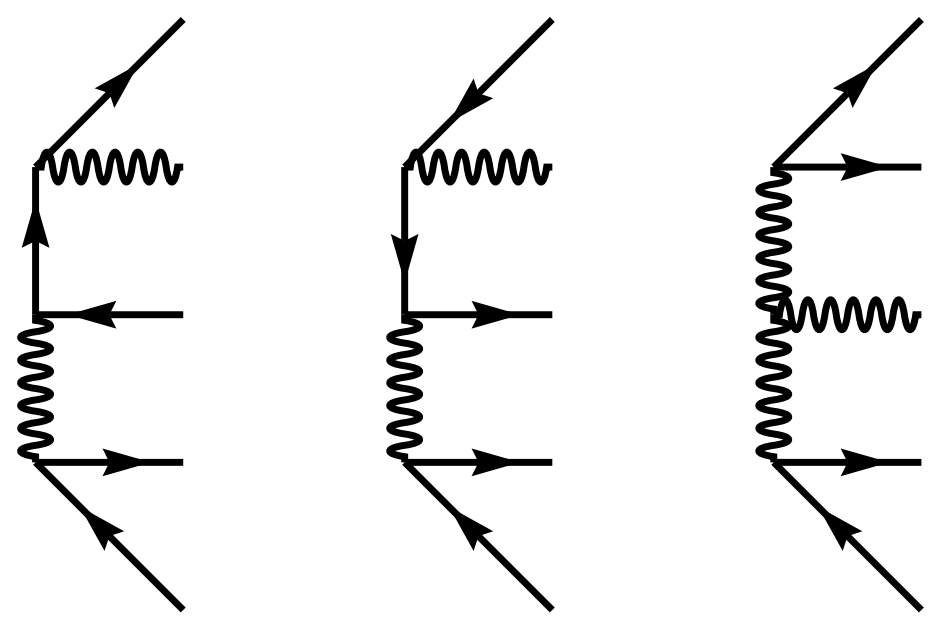

Fig. 4 

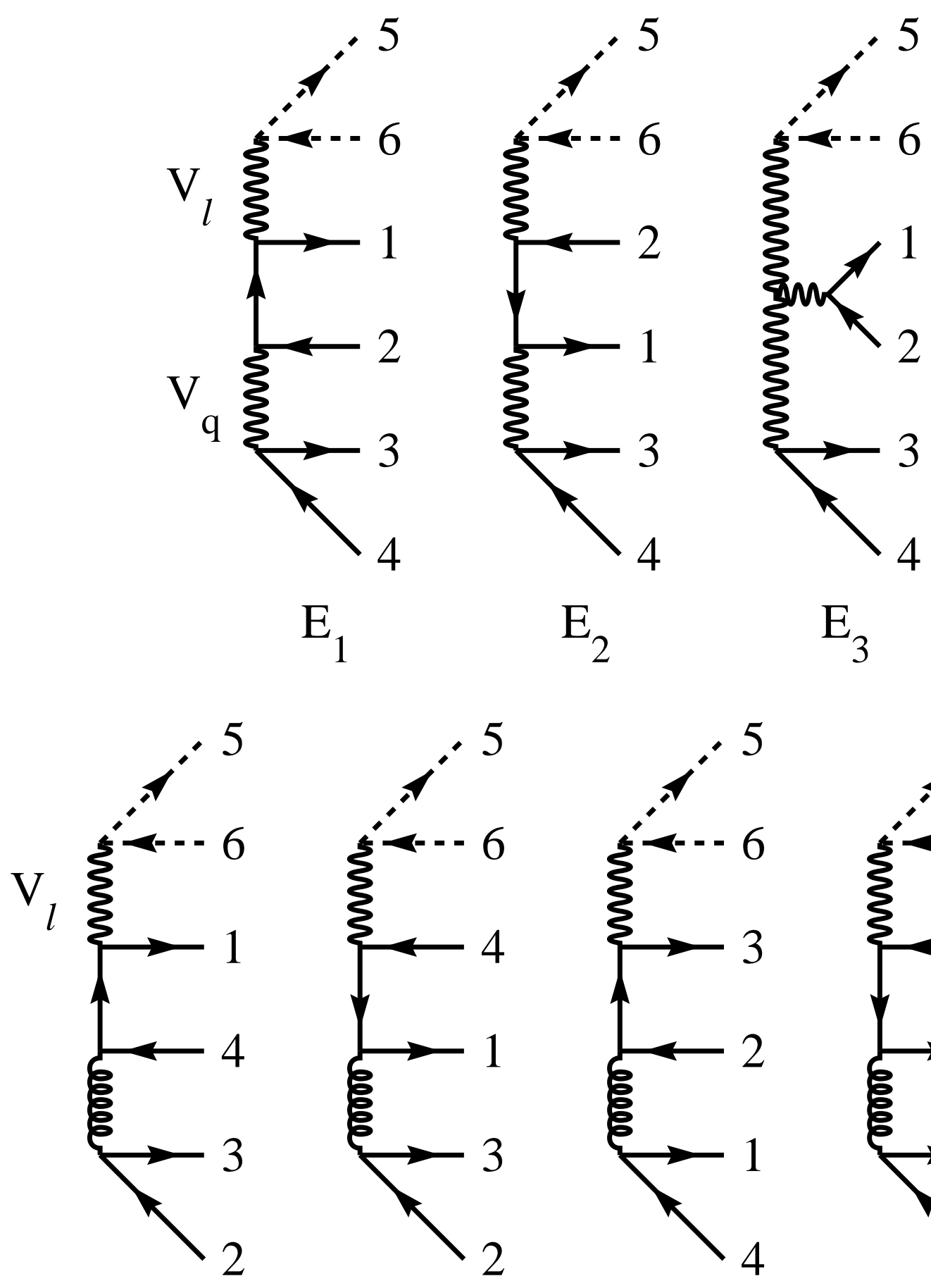

$\mathrm{G}_{1}$

$\mathrm{G}_{2}$

$\mathrm{G}_{3}$

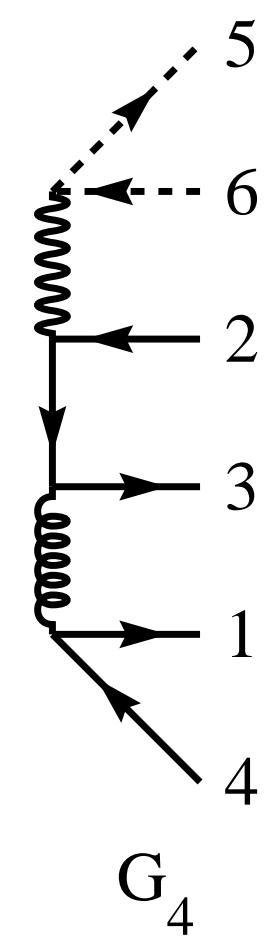

Fig. 5 


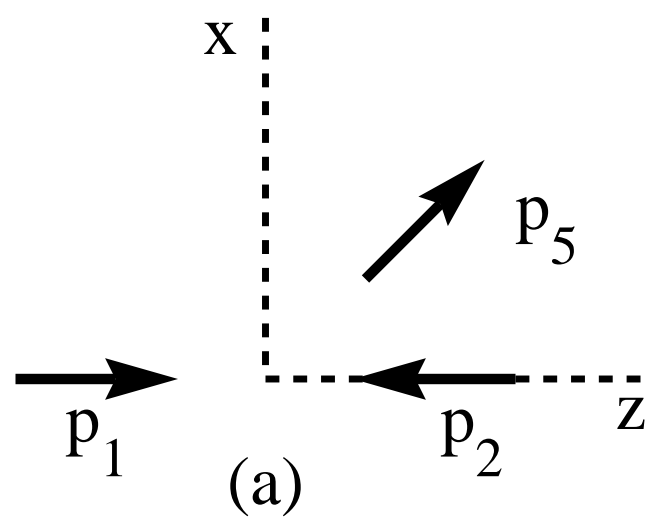

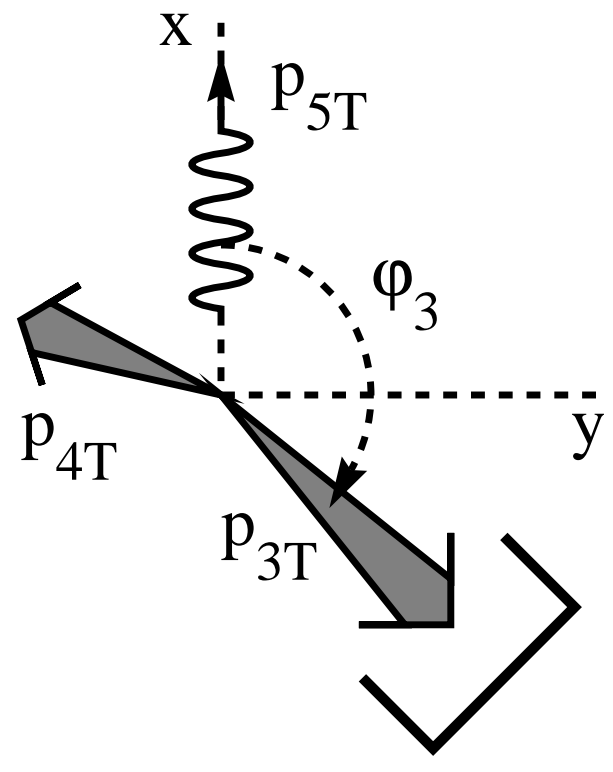

bin

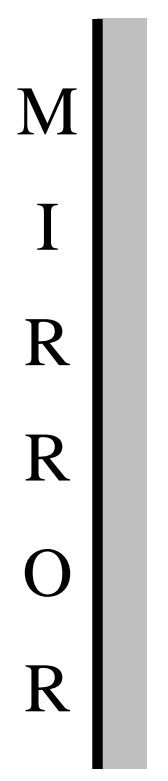

(b)

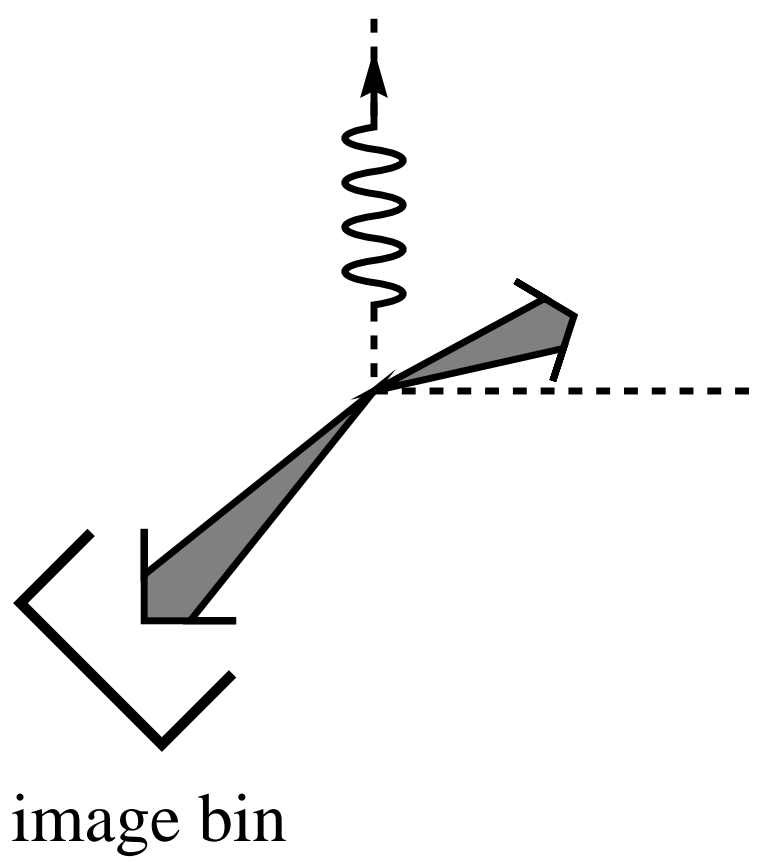

image bin

Fig. 6 

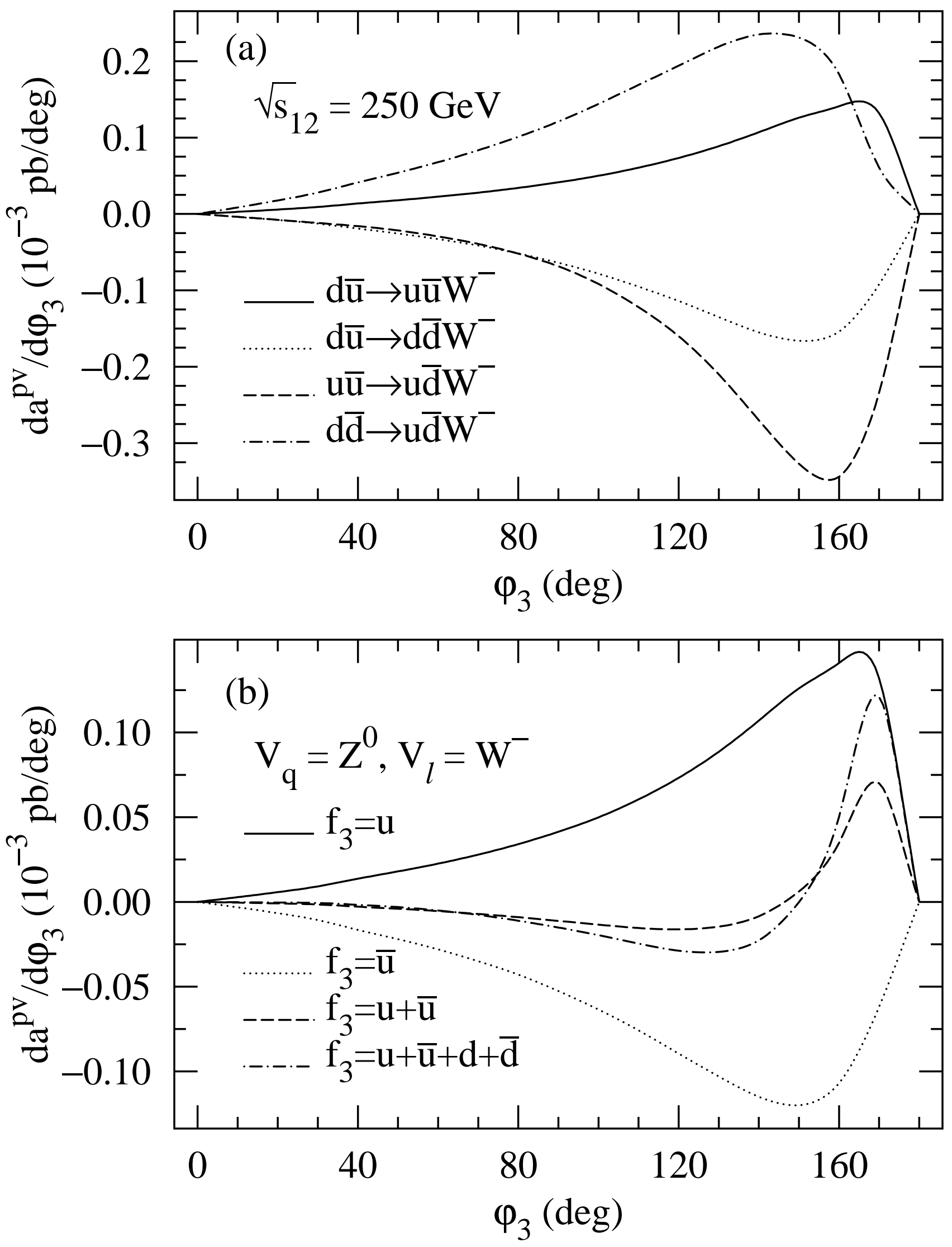

Fig. 7 

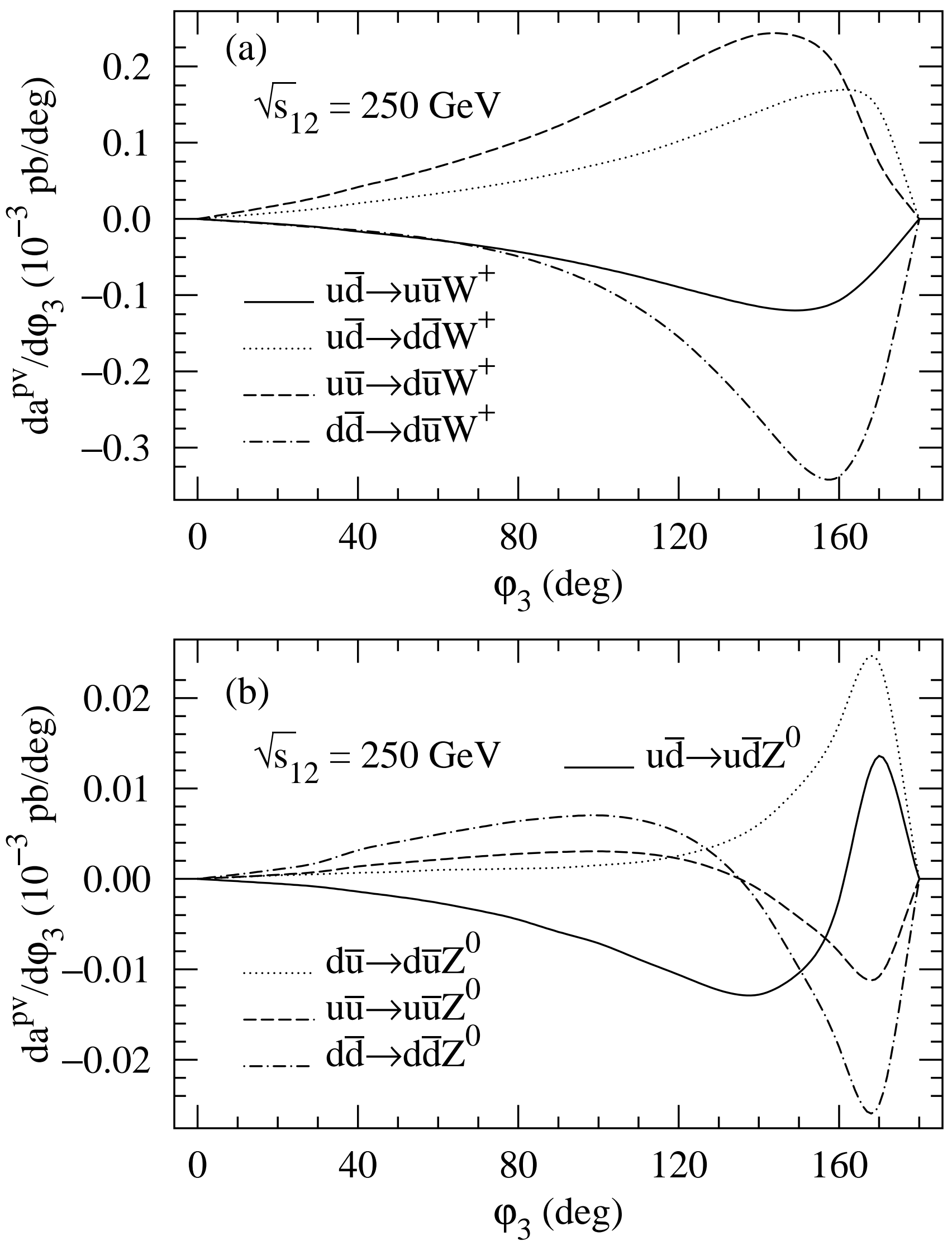

Fig. 8 

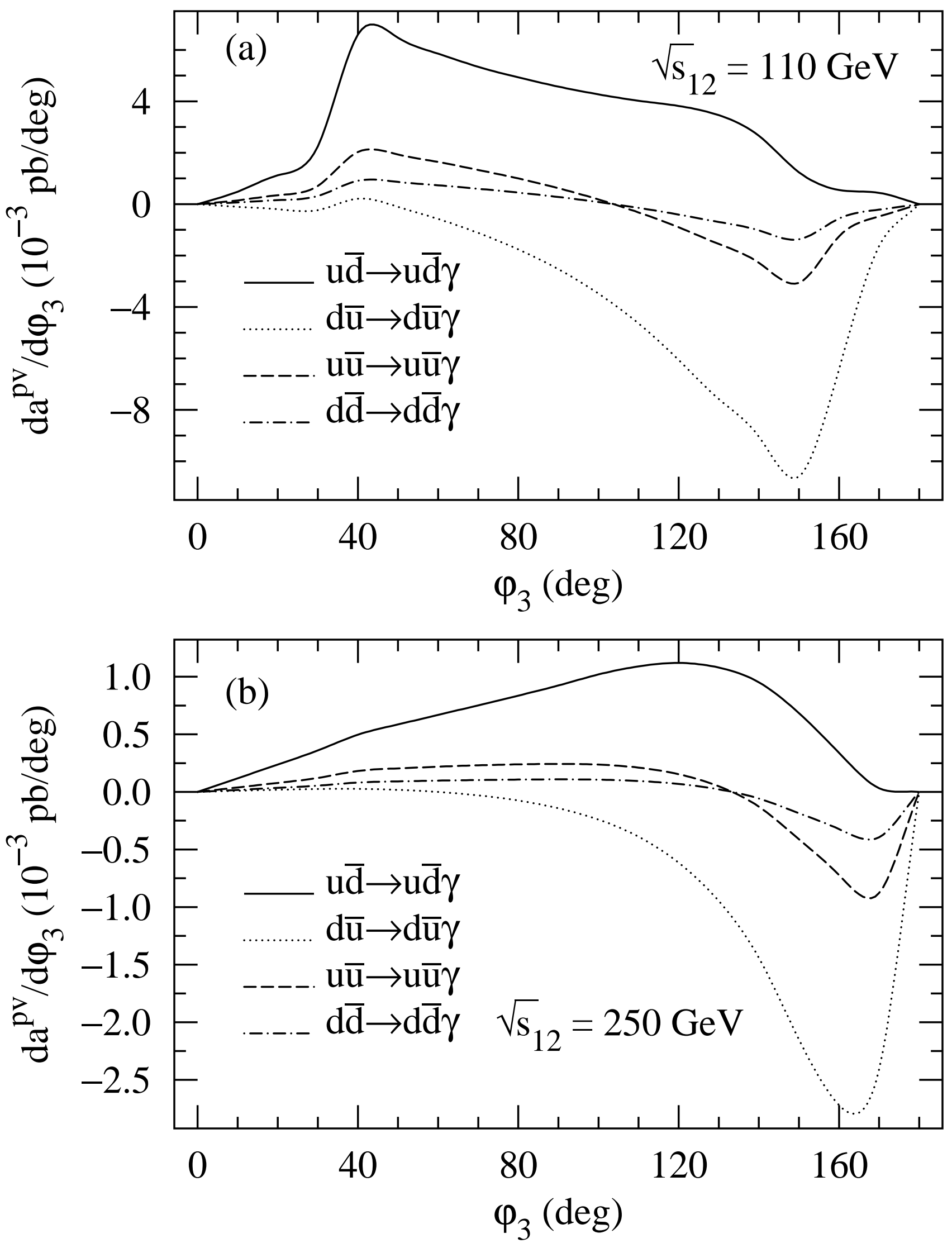

Fig. 9 

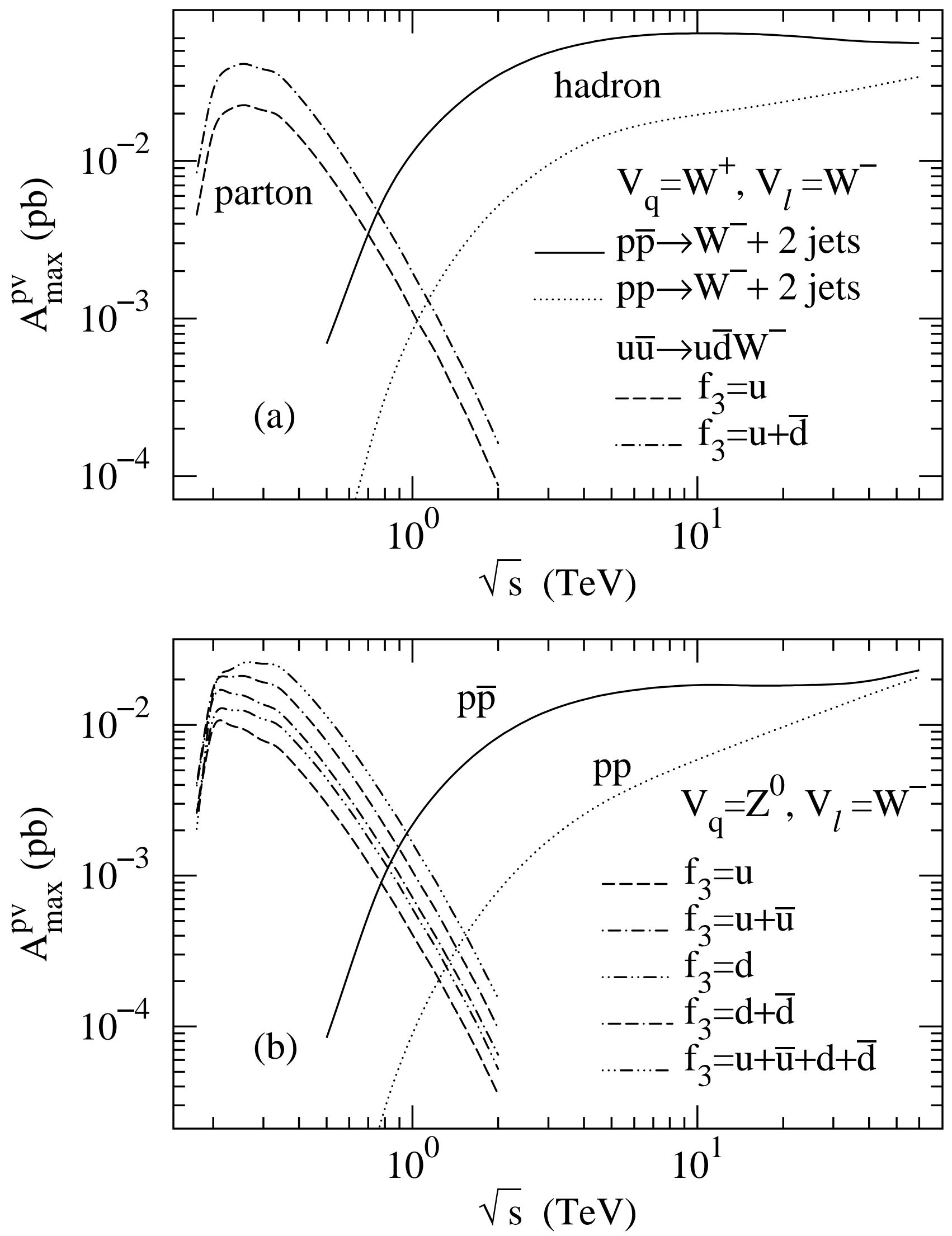

Fig. 10 

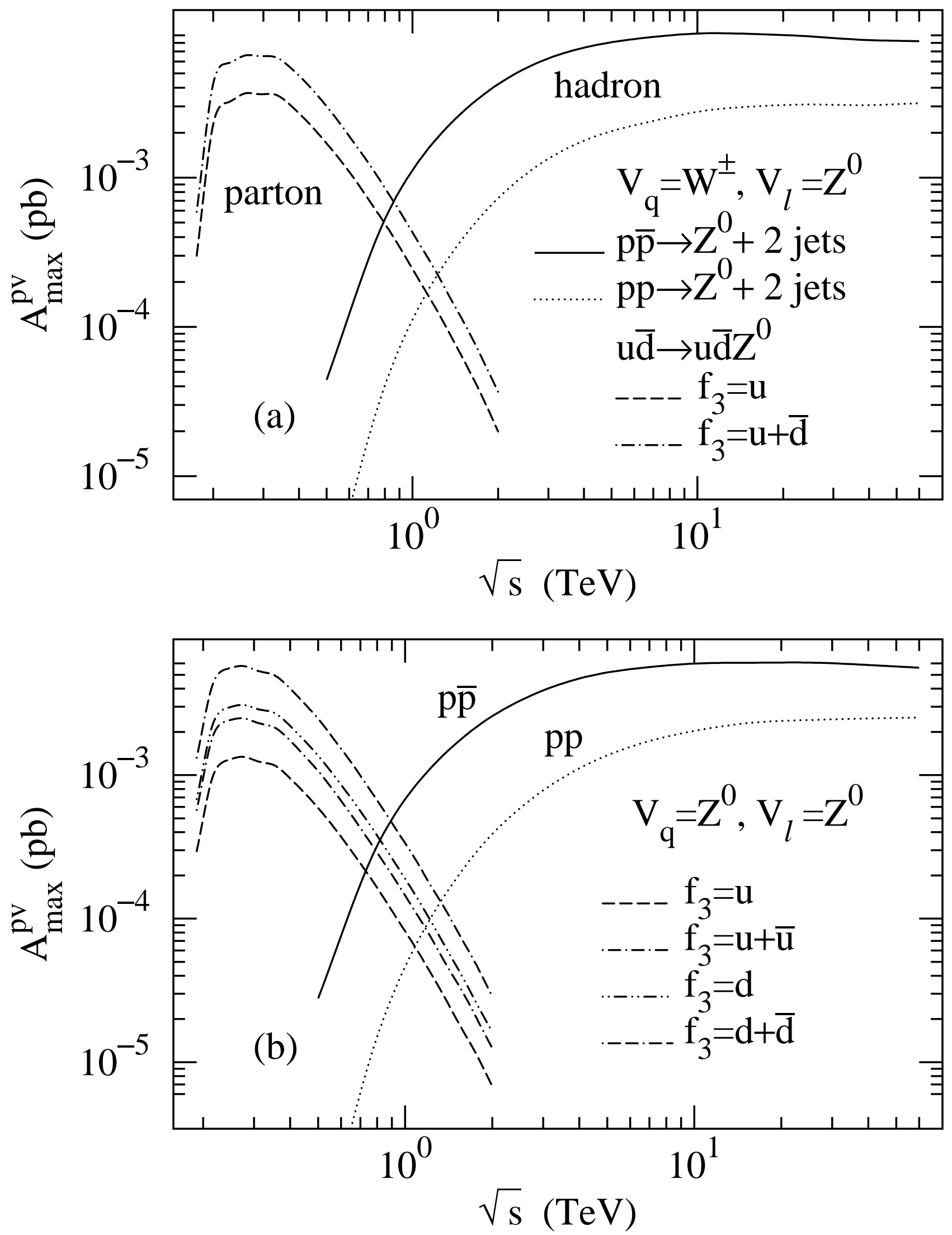

Fig. 11 

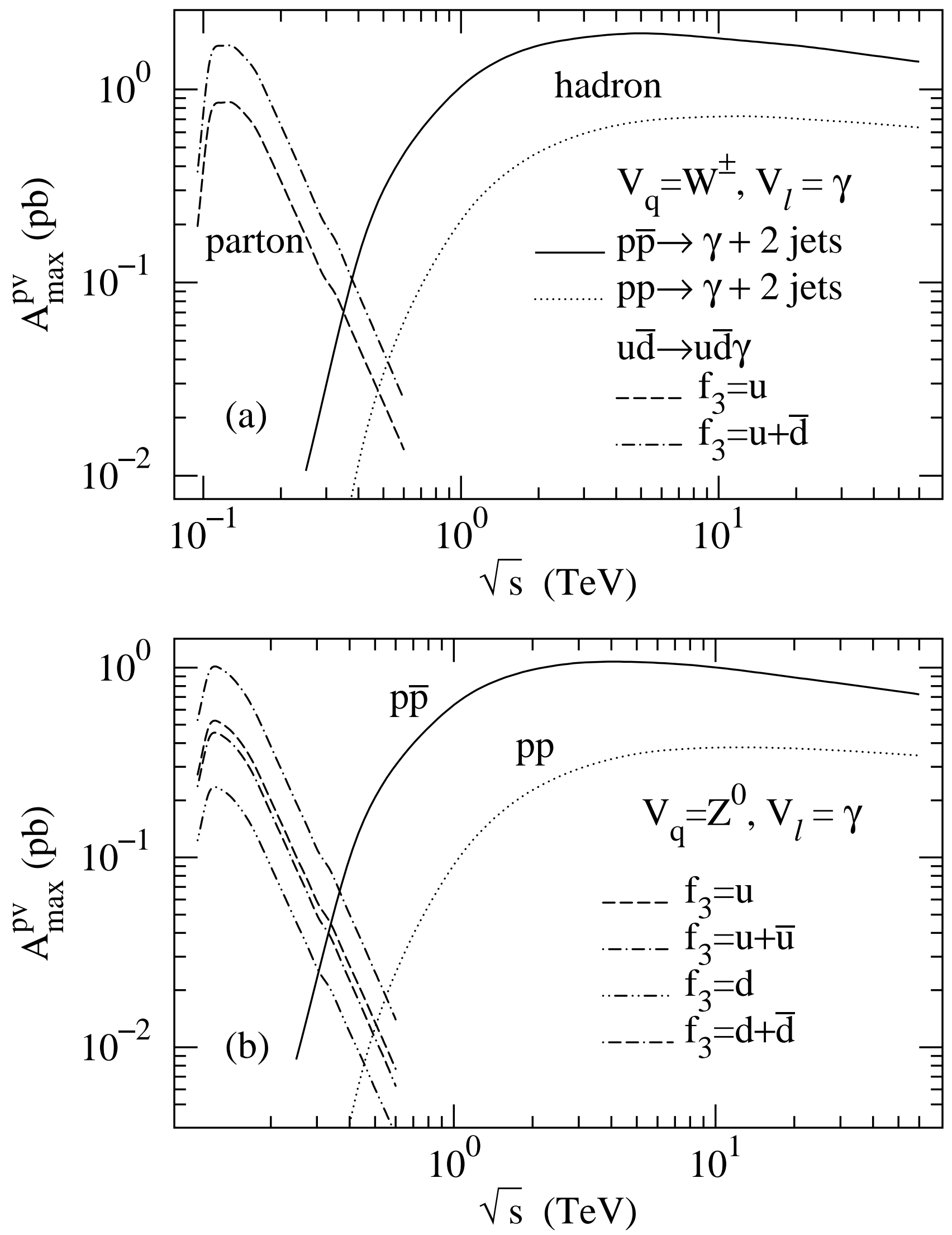

Fig. 12 

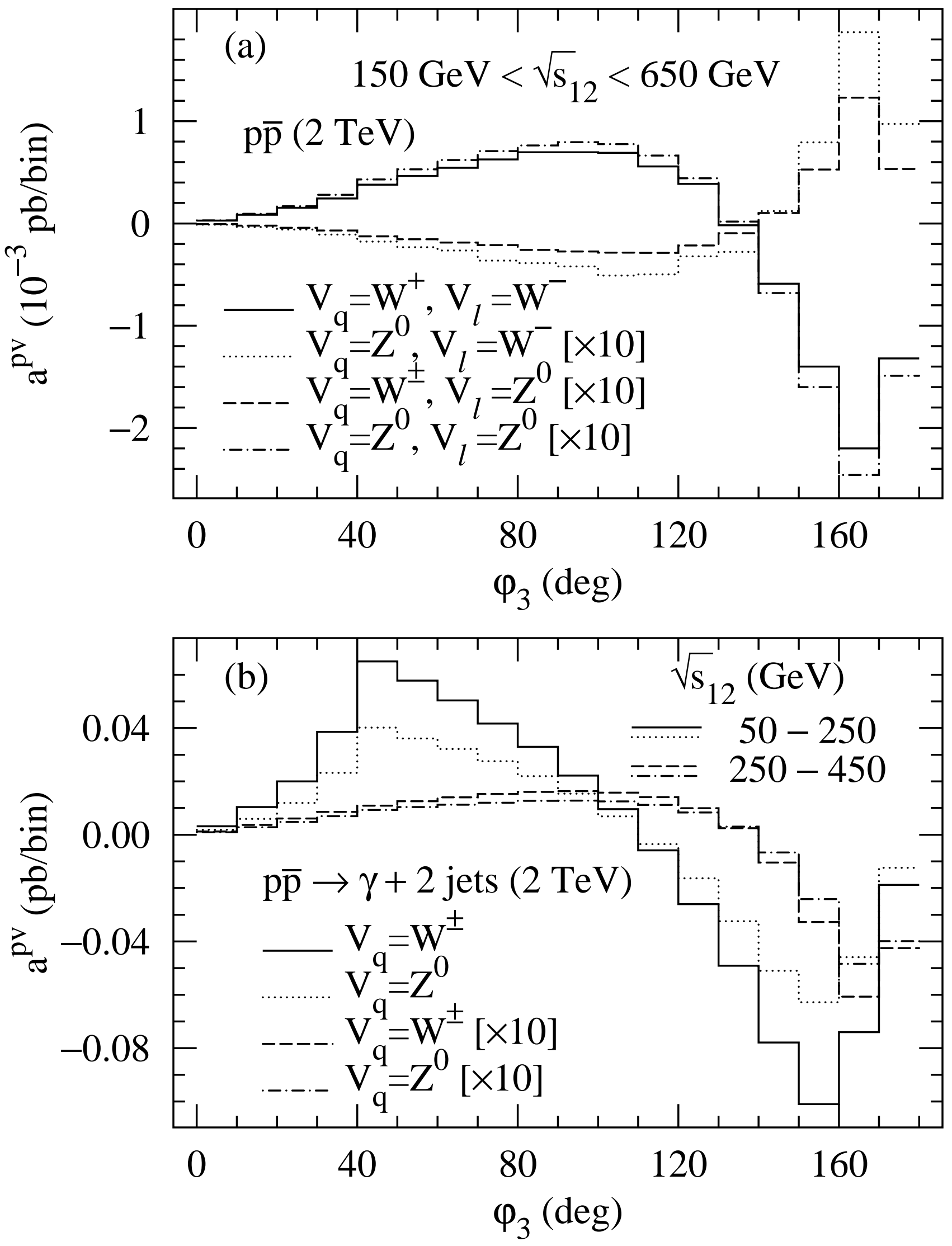

Fig. 13 

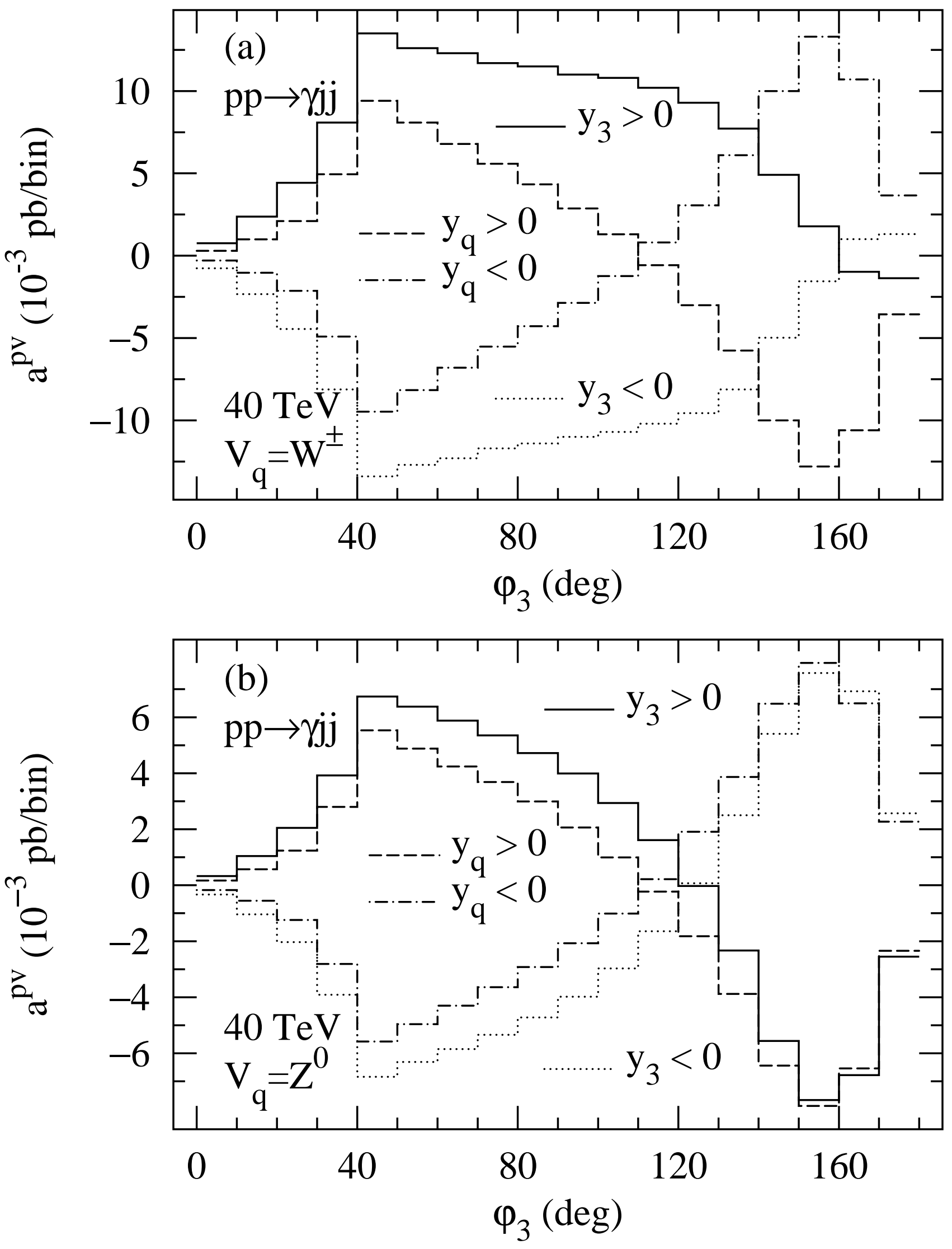

Fig. 14 

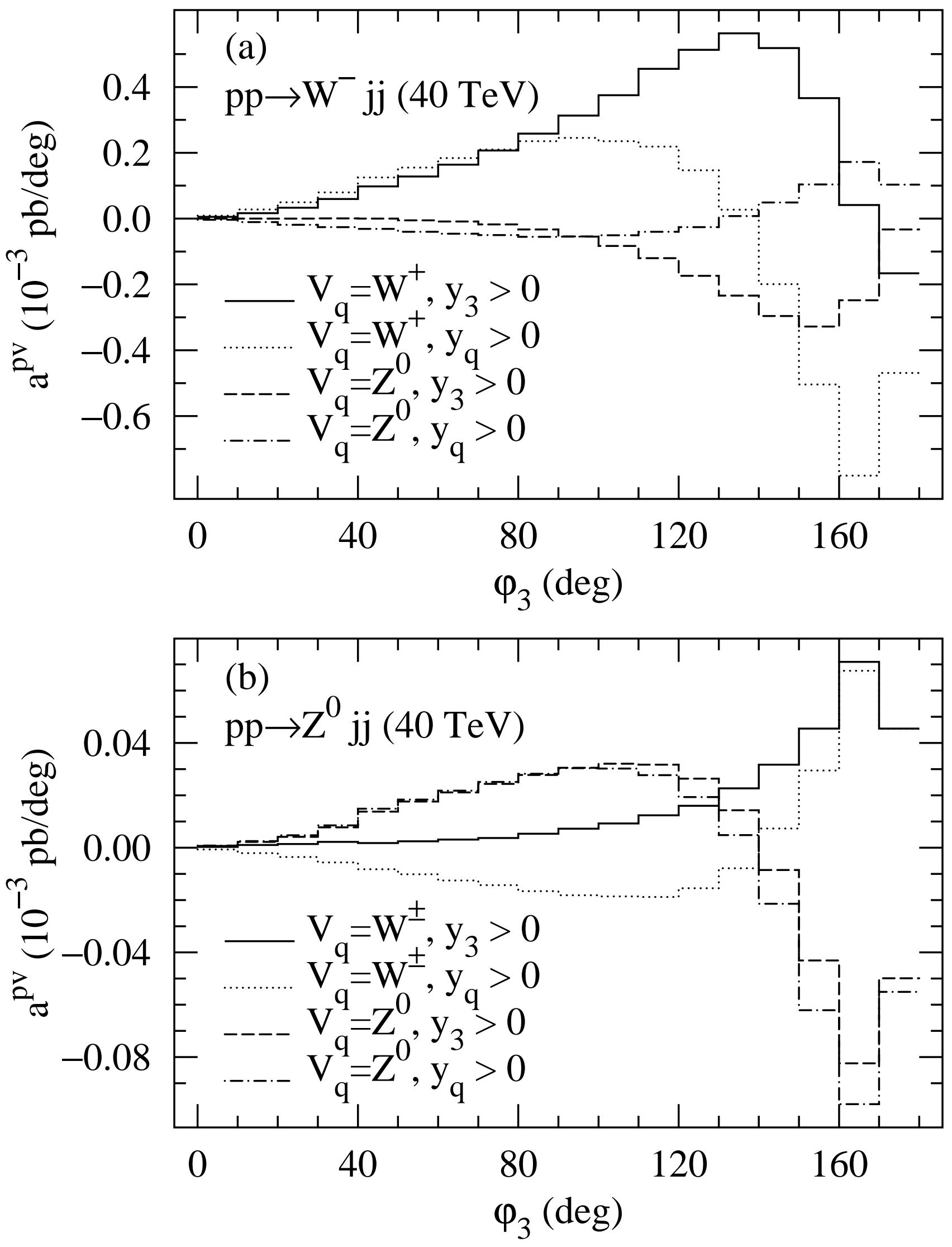

Fig. 15 

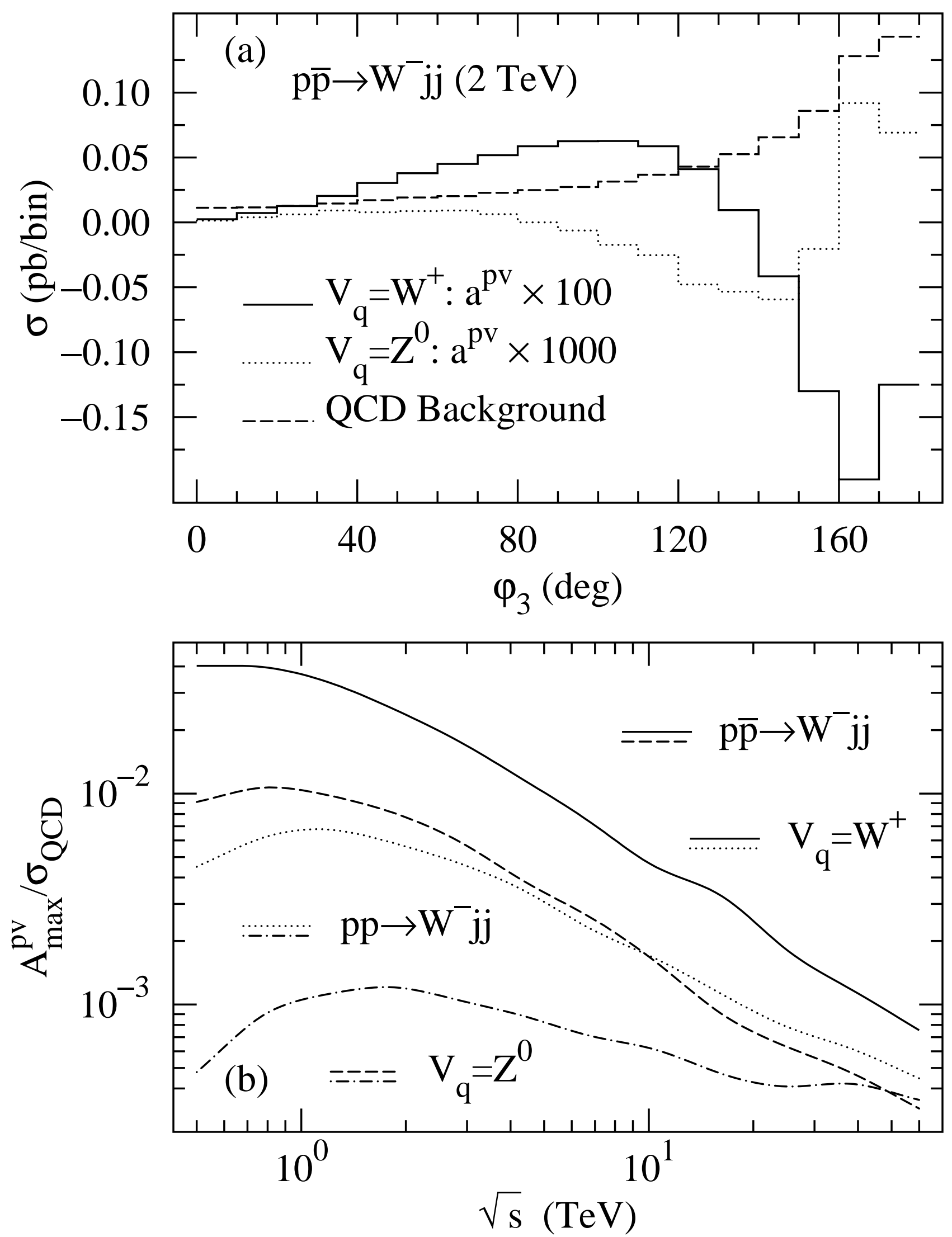

Fig. 16 

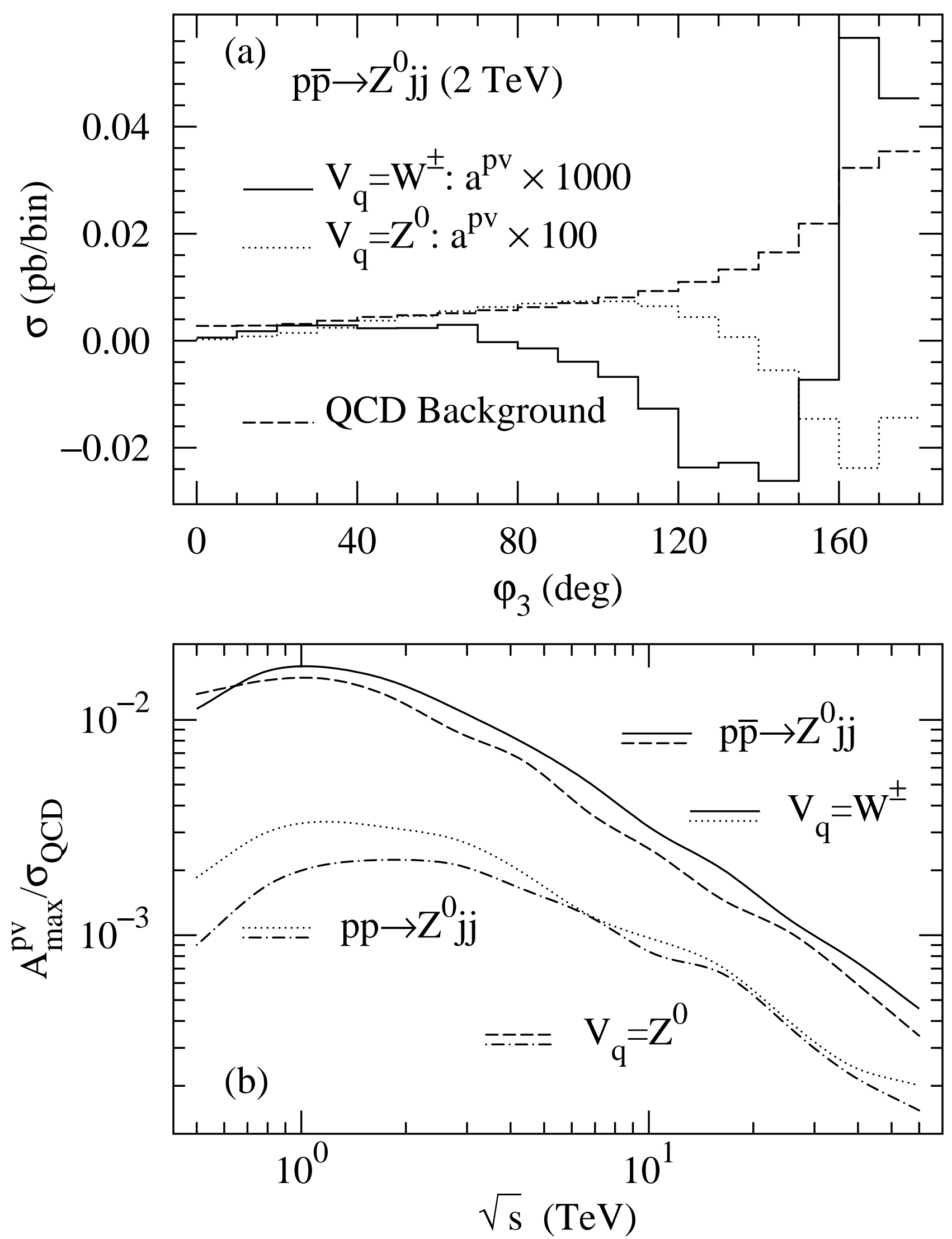

Fig. 17 

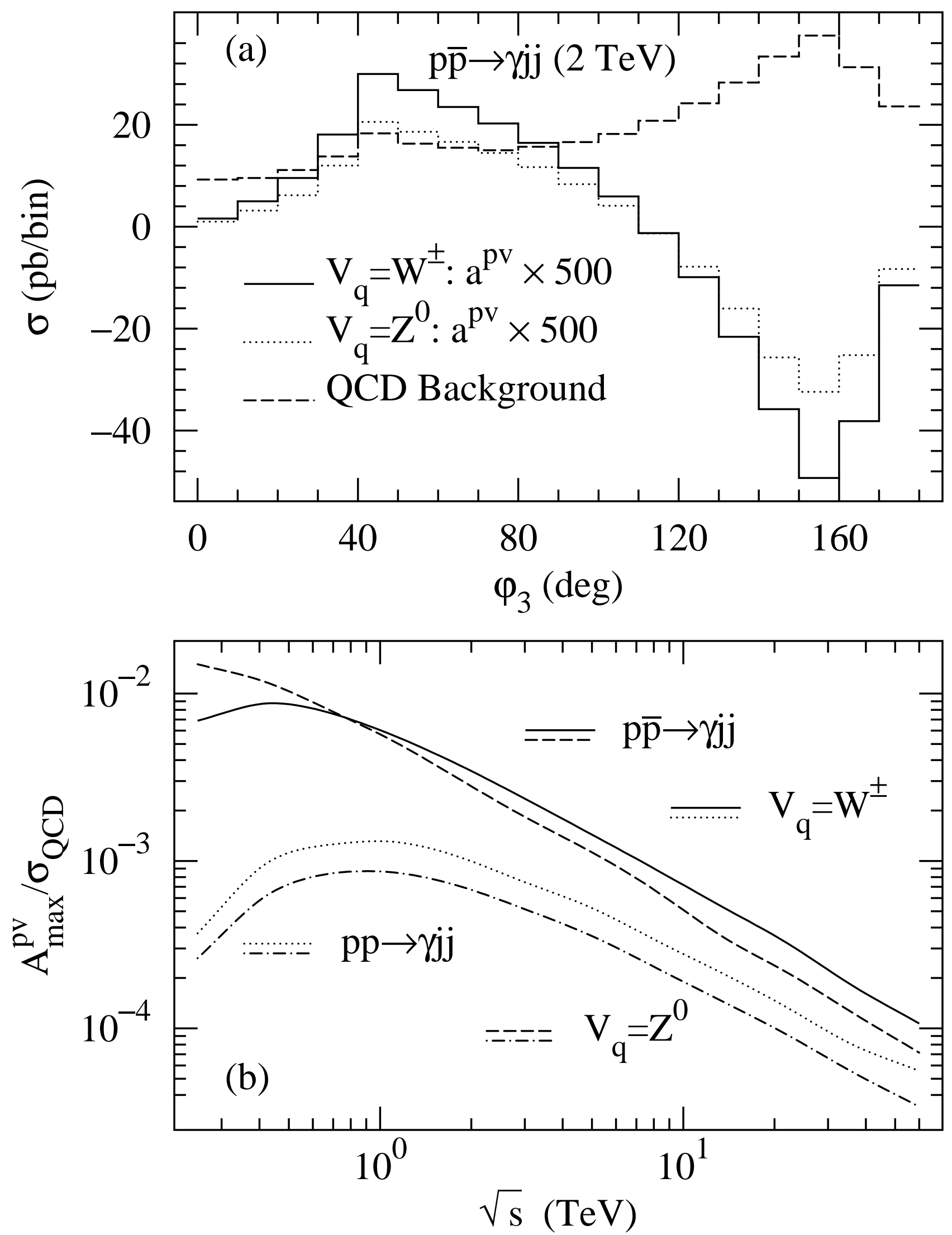

Fig. 18 

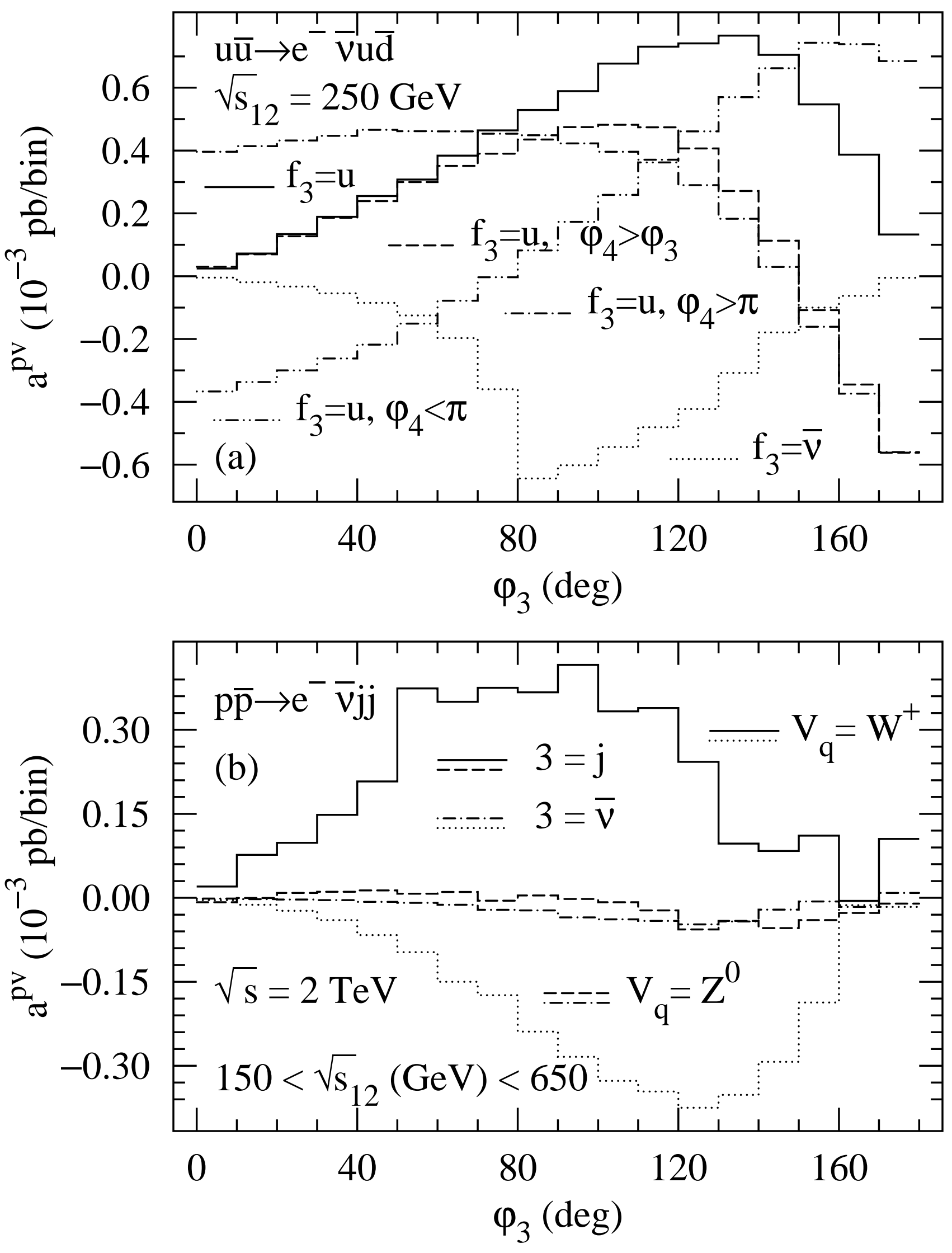

Fig. 19 

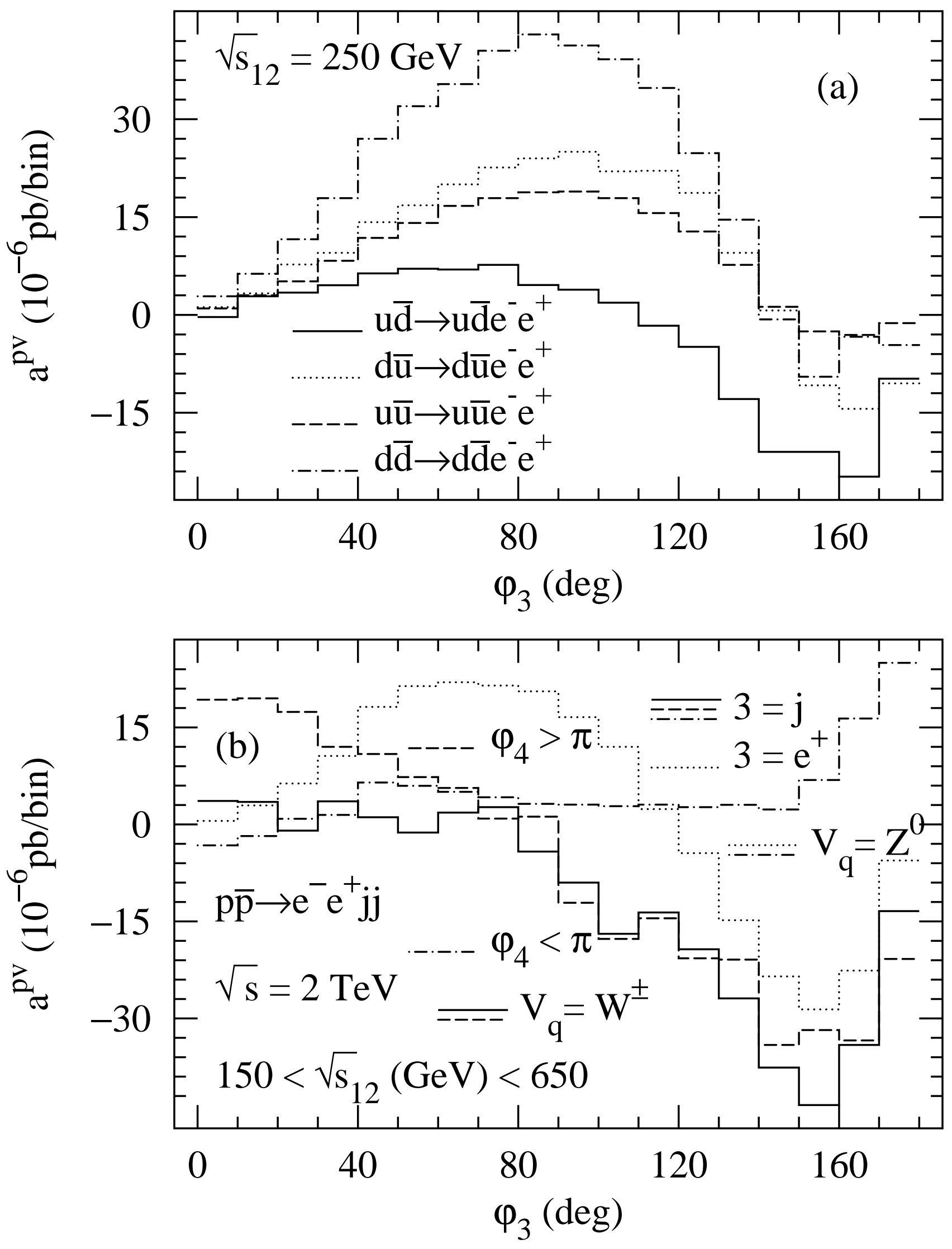

Fig. 20 